\title{
Indefinite Einstein metrics on simple Lie groups
}

\author{
Andrzej Derdzinski and Światosław R. Gal \\ Dedicated to Professor Witold Roter on the occasion of his eightieth birthday
}

\begin{abstract}
The set $\mathcal{E}$ of Levi-Civita connections of left-invariant pseudo-Riemannian Einstein metrics on a given semisimple Lie group always includes D, the Levi-Civita connection of the Killing form. For the groups $\mathrm{SU}(l, j)$ (or $\mathrm{SL}(n, \mathbb{R})$, or $\mathrm{SL}(n, \mathbb{C})$ or, if $n$ is even, $\operatorname{SL}(n / 2, \mathbb{H})$ ), with $0 \leq j \leq l$ and $j+l>2$ (or, $n>2$ ), we explicitly describe the connected component $\mathcal{C}$ of $\mathcal{E}$, containing $\mathrm{D}$. It turns out that $\mathcal{C}$, a relatively-open subset of $\mathcal{E}$, is also an algebraic variety of real dimension $2 l j$ (or, real/complex dimension $\left[n^{2} / 2\right]$ or, respectively, real dimension $4\left[n^{2} / 8\right]$ ), forming a union of $(j+1)(j+2) / 2$ (or, $[n / 2]+1$ or, respectively, $[n / 4]+1)$ orbits of the adjoint action. In the case of $\mathrm{SU}(n)$ one has $2 l j=0$, so that a positive-definite multiple of the Killing form is isolated among suitably normalized left-invariant Riemannian Einstein metrics on $\mathrm{SU}(n)$.
\end{abstract}

Mathematics Subject Classification (2010). 53C30, 53C50, 22E99.

Keywords. Indefinite Einstein metrics, left-invariant Einstein metrics.

\section{Introduction}

The Killing form $\beta$ of any semisimple real Lie group $G$ is a bi-invariant pseudo-Riemannian Einstein metric on the underlying manifold of G. In the case of compact simple groups $\mathrm{G}$ other than $\mathrm{SU}(2)$ and $\mathrm{SO}(3)$, D'Atri and Ziller [6] proved, over three decades ago, the existence on $\mathrm{G}$ of at least one left-invariant Riemannian Einstein metric which is not a multiple of $\beta$. Recently, Gibbons, Lü and Pope [8] found six more such essentially different examples - two on $\mathrm{SO}(5)$ and four on $\mathrm{G}_{2}$. In addition, among left-invariant Einstein metrics on $\mathrm{G}=\mathrm{SU}(3)$, they exhibited one which is indefinite.

This raises the more general question of

(*) classifying left-invariant pseudo-Riemannian Einstein metrics on simple Lie groups.

For noncompact groups, question $(*)$ is in turn related to a conjecture of Alekseevsky [1], cf. [3, p. 190], according to which a noncompact homogeneous space G/K may carry a nonflat G-invariant Riemannian Einstein metric only if $\mathrm{K}$ is a maximal compact subgroup of G. If true, Alekseevsky's conjecture, applied to a noncompact simple group $\mathrm{G}$ and its trivial subgroup $\mathrm{K}$, would imply that left-invariant Einstein metrics on $G$ are all indefinite.

The Einstein condition is essential here, as opposed to mere negativity of the Ricci curvature Rc: in fact, Leite and Dotti [13] constructed (non-Einstein) 
A. Derdzinski and Ś. R. Gal

left-invariant Riemannian metrics with $\mathrm{Rc}<0$ on $\mathrm{SL}(n, \mathbb{R}), n \geq 3$. Also, all noncompact Lie groups known to admit left-invariant Riemannian Einstein metrics are solvable. Heber [9] and Lauret [11] made substantial progress towards understanding the moduli and properties of such metrics on solvable Lie groups.

Given a Lie group $\mathrm{G}$ with the Lie algebra $\mathfrak{g}$, let $\mathcal{E}$ denote the set of all Einstein connections in $\mathfrak{g}$, by which we mean the Levi-Civita connections of left-invariant pseudo-Riemannian Einstein metrics on G. One may view $\mathcal{E}$ as a set of bilinear operations $\mathfrak{g} \times \mathfrak{g} \rightarrow \mathfrak{g}$, that is, a subset of $\left[\mathfrak{g}^{*}\right]^{\otimes 2} \otimes \mathfrak{g}$. If $\mathfrak{g}$ is semisimple, the Levi-Civita connection $\mathrm{D}=[] /$,2 of the Killing form $\beta$ is an element of $\mathcal{E}$.

The present paper provides an initial step towards answering the question stated above in $(*)$ : for any simple Lie algebra $\mathfrak{g}$, we explicitly describe the connected component $\mathcal{C}$ of $\mathcal{E}$ containing D. As $\mathcal{C}$ turns out to be a relatively open subset of $\mathcal{E}$, it also contains all Einstein connections sufficiently close to D.

We restrict much of our discussion to $\mathfrak{g}$ that correspond to the groups

$$
\mathrm{G}=\mathrm{SL}(n, \mathbb{R}), \mathrm{G}=\mathrm{SL}(n, \mathbb{C}), \mathrm{G}=\mathrm{SU}(l, j) \text {, or }(n \text { even }) \mathrm{G}=\mathrm{SL}(n / 2, \mathbb{H}),
$$

with $l \geq j \geq 0$ and $n=l+j \geq 3$ since, for all remaining simple Lie algebras, D is isolated in $\mathcal{E}$ and $\mathcal{C}=\{\mathrm{D}\}$. (See Remark 12.3.) Our main result, Theorem 22.2, realizes $\mathcal{C}$, for $\mathrm{G}$ in (1.1), as the bijective image of a well-understood algebraic set in $\mathfrak{g}$ under a specific nonhomogeneous quadratic mapping $\mathfrak{g} \rightarrow\left[\mathfrak{g}^{*}\right]^{\otimes 2} \otimes \mathfrak{g}$. Theorem 22.2 also states that $\mathcal{C}$ itself is an algebraic set of dimension $d_{\mathbb{F}}$ over $\mathbb{F}$, consisting of $s$ orbits of the adjoint action, where $\left(d_{\mathbb{F}}, s\right)=\left(\left[n^{2} / 2\right],[n / 2]+1\right)$ for $\mathrm{G}=\mathrm{SL}(n, \mathbb{F})$ and $\left(d_{\mathbb{R}}, s\right)=([n / 2]+1,(j+1)(j+2) / 2)$ if $\mathrm{G}=\mathrm{SU}(l, j)$, while $\left(d_{\mathbb{R}}, s\right)=\left(4\left[n^{2} / 8\right],[n / 4]+1\right)$ when $\mathrm{G}=\mathrm{SL}(n / 2, \mathbb{H})$.

As a consequence (Theorem 22.3), on SU(n), positive-definite multiples of the Killing form are isolated among left-invariant Riemannian Einstein metrics. This is a special case of a conjecture made by Böhm, Wang and Ziller [4, p. 683].

For the group $\mathrm{G}=\mathrm{SL}(n, \mathbb{C})$ our argument leads to the following conclusion (Theorem 22.4): all left-invariant pseudo-Riemannian Einstein metrics on G close to multiples of the Killing form are real parts of holomorphic Einstein metrics, cf. [12, p. 210], which also makes them Kähler-Norden metrics in the sense of [16].

\section{Outline of the main argument}

Let $\mathrm{G}$ be a real or complex semisimple Lie group, with the associated semisimple Lie algebra $\mathfrak{g}$ over $\mathbb{F}=\mathbb{R}$ or $\mathbb{F}=\mathbb{C}$. We study left-invariant connections on $G$, which are also holomorphic when $\mathbb{F}=\mathbb{C}$, as well as left-invariant metrics on $\mathrm{G}$, assuming the latter to be pseudo-Riemannian if $\mathbb{F}=\mathbb{R}$, and holomorphic (in the sense of being $\mathbb{C}$-bilinear, symmetric and nondegenerate) if $\mathbb{F}=\mathbb{C}$.

In this section we skip the term 'left-invariant' and simply speak of connections in $\mathfrak{g}$ and metrics in $\mathfrak{g}$. An example of the latter is the Killing form $\beta$. 
All vector spaces under consideration are finite-dimensional. The spaces

$$
\mathcal{T}, \quad \mathcal{Y}, \quad \mathcal{S}, \quad \text { with } \mathcal{S} \subset \mathcal{Y}
$$

consist, respectively, of symmetric $\mathbb{F}$-bilinear forms $\mathfrak{g} \times \mathfrak{g} \rightarrow \mathbb{F}$ (such as metrics in $\mathfrak{g}$ ), arbitrary connections in $\mathfrak{g}$ (treated as $\mathbb{F}$-bilinear operations $\mathfrak{g} \times \mathfrak{g} \rightarrow \mathfrak{g}$ ) and, finally, the operations $\mathfrak{g} \times \mathfrak{g} \rightarrow \mathfrak{g}$ in $\mathcal{Y}$ which are symmetric. Thus, $\beta \in \mathcal{T}$ for the Killing form $\beta$, and $\mathrm{D} \in \mathcal{Y}$, where $\mathrm{D}=[] /$,2 is the standard connection. Our discussion focuses on the affine subspace $\mathrm{D}+\mathcal{S}$ of $\mathcal{Y}$ formed by connections $\nabla \in \mathcal{Y}$ which are torsion-free, so that their skew-symmetric part is $\mathrm{D}$. We refer to any such $\nabla=\mathrm{D}+\mathrm{S}$, with $\mathrm{S} \in \mathcal{S}$, as a weakly-Einstein connection in $\mathfrak{g}$, if the symmetric 2-tensor $\{\nabla \cdot \nabla\}$ defined in Section 9, is $\nabla$-parallel, that is,

$$
\nabla\{\nabla \cdot \nabla\}=0
$$

Since $\mathfrak{g}$ is semisimple, whenever $\nabla$ happens to be the Levi-Civita connection of a metric, its Ricci tensor is $\rho^{\nabla}=-\{\nabla \cdot \nabla\}$. The set $\mathcal{W}$ of all weakly-Einstein connections in $\mathfrak{g}$ thus contains the set $\mathcal{E}$ of Einstein connections in $\mathfrak{g}$, defined here to be the Levi-Civita connections $\nabla$ of (left-invariant) Einstein metrics $\gamma$ on G. By the latter we mean, as usual, all $\gamma$ with $\rho^{\nabla}=\kappa \gamma$ for some $\kappa \in \mathbb{F}$.

For instance, D is an Einstein connection, being the Levi-Civita connection of the Einstein metric $\beta$ (the Killing form).

The converse "weakly-Einstein implies Einstein" of the implication "Einstein implies weakly-Einstein" $(\mathcal{E} \subset \mathcal{W})$, generally false (Section 29$)$, is true generically:

$$
\text { if } \nabla \in \mathcal{W} \text { and }\{\nabla \cdot \nabla\} \text { is nondegenerate, then } \nabla \in \mathcal{E} \text {. }
$$

In fact, $\nabla$ then is the Levi-Civita connection of the metric $\{\nabla \cdot \nabla\}$, with the Ricci tensor $\rho^{\nabla}=-\{\nabla \cdot \nabla\}$.

Instead of Einstein metrics, we choose to look for weakly-Einstein connections in $\mathfrak{g}$. By (2.2), the latter question is only slightly more general than the former. It can, however, be phrased in much simpler algebraic terms: for $\boldsymbol{H}: \mathcal{S} \rightarrow \mathcal{S}$ given by $\boldsymbol{H}(\mathrm{S})=4(\mathrm{D}+\mathrm{S})\{(\mathrm{D}+\mathrm{S}) \cdot(\mathrm{D}+\mathrm{S})\}$ (the left-hand side in $(2.1)$ with $\nabla=\mathrm{D}+\mathrm{S})$

$$
\mathcal{W}=\{\nabla \in \mathrm{D}+\mathcal{S}: \nabla\{\nabla \cdot \nabla\}=0\}=\mathrm{D}+\boldsymbol{H}^{-1}(0)
$$

while $\boldsymbol{H}$ is a (nonhomogeneous) cubic polynomial mapping, due to the quadratic dependence of $\{\nabla \cdot \nabla\}$ on $\nabla$.

Enlarging the space of unknowns, we rewrite the cubic condition $\boldsymbol{H}(\mathrm{S})=0$ as a system of quadratic equations. Specifically (Remark 11.3), there exists

a bijective correspondence between $\mathcal{W}=\mathrm{D}+\boldsymbol{H}^{-1}(0)$

and the set of all $(\mathrm{S}, \sigma) \in \mathcal{S} \times \mathcal{T}$ with $\boldsymbol{K}(\mathrm{S}, \sigma)=(0,0)$,

where $\boldsymbol{K}: \mathcal{S} \times \mathcal{T} \rightarrow \mathcal{S} \times \mathcal{T}$ is a suitable nonhomogeneous quadratic mapping. 
Thus, since weakly-Einstein connections (elements of $\mathcal{W}$ ) are precisely those $\mathrm{D}+\mathrm{S}$ for which $\mathrm{S} \in \mathcal{S}$ and $\boldsymbol{H}(\mathrm{S})=0$,

the search for weakly-Einstein connections $\mathrm{D}+\mathrm{S}$ is reduced to solving the equation $\boldsymbol{K}(\mathrm{S}, \sigma)=(0,0)$ for $\mathrm{S} \in \mathcal{S}$ and $\sigma \in \mathcal{T}$.

From now on, $\mathfrak{g}$ is assumed to be one of the simple Lie algebras

$$
\mathfrak{s l}(n, \mathbb{R}), \mathfrak{s l}(n, \mathbb{C}), \mathfrak{s u}(l, j) \text { with } l \geq j \geq 0 \text { and } n=l+j \geq 3 \text {, or } \mathfrak{s l}(n / 2, \mathbb{H}),
$$

the last for even $n$ only. As a first step towards constructing $(\mathrm{S}, \sigma) \in \mathcal{S} \times \mathcal{T}$ with $\boldsymbol{K}(\mathrm{S}, \sigma)=(0,0)$, we set $(\mathrm{S}, \sigma)=(\mathrm{D} \lambda, \psi)$, where $\lambda, \psi$ are certain special elements of $\mathcal{T}$ and $\mathrm{D} \lambda$ is the D-covariant derivative of $\lambda$ (treated as an element of $\mathcal{S}$ via $\beta$-index-raising). More precisely, we define in Section 16 some $\tau_{a}, \theta_{a}, \mu_{a} \in \mathcal{T}$, which depend on $a \in \mathfrak{g}$, then choose $\lambda=x \tau_{a}+n^{2} y \theta_{a}+z \mu_{a}$ and $\psi=p \tau_{a}+q \theta_{a}+r \mu_{a}+f \beta$ with (unknown) scalar parameters $f, x, y, z, p, q, r$. In other words,

$$
\begin{aligned}
& (\mathrm{D} \lambda, \psi)=\boldsymbol{I}_{a}[\mathbf{v}] \text { for the linear operator } \mathbf{I}_{a}: \mathbb{F}^{9} \rightarrow \mathcal{S} \times \mathcal{T} \text { given by } \\
& \mathbf{I}_{a}[\mathbf{v}]=\left(x \mathrm{D} \tau_{a}+n^{2} y \mathrm{D} \theta_{a}+z \mathrm{D} \mu_{a}, p \tau_{a}+q \theta_{a}+r \mu_{a}+f \beta\right)
\end{aligned}
$$

and any $\mathbf{v}=(\xi, f, h, x, y, z, p, q, r) \in \mathbb{F}^{9}$. The variables $\xi$ and $h$, not occurring in the formula, become useful later. Note that, if $\mathbf{v}=(\xi, f, h, x, y, z, p, q, r)$,

$$
\mathbf{I}_{b}[\mathbf{v}]=\mathbf{0} \text { whenever } b=0 \text { and } f=0 .
$$

To find $a, \mathbf{v}$ with $\boldsymbol{K}\left(\mathbf{I}_{a}[\mathbf{v}]\right)=(0,0)$, we prove in Section 21 the fundamentalformula

$$
\boldsymbol{K}\left(\boldsymbol{I}_{a}[\mathbf{v}]\right)-\boldsymbol{I}_{a}[\boldsymbol{J}(\mathbf{v})]-\boldsymbol{I}_{b}[\boldsymbol{M}(\mathbf{v})] \in \mathcal{N}
$$

for any $(a, \mathbf{v}) \in \mathfrak{g} \times \mathbb{F}^{9}$, where $\boldsymbol{J}, \boldsymbol{M}$ are specific polynomial mappings $\mathbb{F}^{9} \rightarrow \mathbb{F}^{9}$. (Remark 21.2 describes $\boldsymbol{J}$ and $\boldsymbol{M}$, while (2.7) appears as (21.2) - (21.3).)

What $\mathcal{N}$ and $\in$ in (2.7) stand for requires the following, more detailed explanation. First, we treat the left-hand side of (2.7) as a polynomial function of

$$
\text { the variables }(a, b, \mathbf{v})=(a, b, \xi, f, h, x, y, z, p, q, r) \in \mathfrak{g} \times \mathfrak{g} \times \mathbb{F}^{9}
$$

with values in $\mathcal{S} \times \mathcal{T}$. This is achieved by replacing all occurrences of

$$
\begin{aligned}
& a^{2} \text { with } \varepsilon^{-1}(h a+b)+\varepsilon^{-2} \xi \mathrm{Id} \text {, which in turn leads to replacements of } \\
& \text { tr } a^{2} \text { by } n \varepsilon^{-2} \xi, \text { and } a^{3} \text { by } \varepsilon^{-1} b a+\varepsilon^{-2}\left(h^{2}+\xi\right) a+\varepsilon^{-2} h b+\varepsilon^{-3} \xi h \mathrm{Id} \text {. }
\end{aligned}
$$

(Here $\varepsilon \in\{1, \mathrm{i}\}$ is a parameter depending on $\mathfrak{g}$, cf. (16.1).) Although $h$ still remains quite artificial, $\xi$ and $b$ have now become more tangible.

Secondly, $\in$ in (2.7) means that the function of (2.8) standing on the lefthand side of (2.7) is itself an element of $\mathcal{N}$ (rather than taking values in $\mathcal{N}$ ).

Finally, $\mathcal{N}$ is the space of $(\mathcal{S} \times \mathcal{T})$-valued negligible functions on $\mathfrak{g} \times \mathfrak{g} \times \mathbb{F}^{9}$. Negligible (polynomial) functions on $\mathfrak{g} \times \mathfrak{g} \times \mathbb{F}^{9}$, valued in various vector spaces, are defined in Section 20; the proper class formed by them (in the sense of set 
theory) may be called an ideal, since one easily verifies that, applying a multilinear mapping to several polynomial functions on $\mathfrak{g} \times \mathfrak{g} \times \mathbb{F}^{9}$, one of which is negligible, we always obtain another negligible function. In addition,

negligible functions vanish at all $(a, b, \mathbf{v}) \in \mathfrak{g} \times \mathfrak{g} \times \mathbb{F}^{9}$ such that $b=0$.

One specific vector $\mathbf{u}=(\xi, f, h, x, y, z, p, q, r) \in \mathbb{F}^{9}$, given by formula (19.3), has

$$
\boldsymbol{J}(\mathbf{u})=\mathbf{0} \text { and } \xi=h=0 .
$$

In Section 22 we use $\mathbf{u}$ to define a family $\mathcal{C}$ of Einstein connections by

$$
\mathcal{C}=\mathrm{D}+\pi_{\mathcal{S}}\left(\left\{\boldsymbol{I}_{a}[\mathbf{u}]: a \in \mathfrak{g} \text { and } a^{2}=0\right\}\right),
$$

which appears as formula $(22.3) ; \pi_{\mathcal{S}}: \mathcal{S} \times \mathcal{T} \rightarrow \mathcal{S}$ is the Cartesian projection.

That every $\nabla=\mathrm{D}+\mathrm{S} \in \mathcal{C}$ is a weakly-Einstein connection (or, equivalently, $\boldsymbol{K}(\mathrm{S}, \sigma)=(0,0)$ for the corresponding $(\mathrm{S}, \sigma)$, cf. (2.3)) is immediate: by (2.9), with $\xi=h=0$ due to (2.11), the relation $a^{2}=0$ in $(2.12)$ gives $b=0$. Now (2.10) implies that the value of left-hand side of $(2.7)$ at $(a, b, \mathbf{u})$ equals 0 , while $\boldsymbol{I}_{b}[\boldsymbol{M}(\mathbf{v})]=0$ by $(2.6)$, since the $f$-component $\boldsymbol{M}(\mathbf{v})$ vanishes identically, and $\boldsymbol{I}_{a}[\boldsymbol{J}(\mathbf{u})]=0$ due to (2.11) and linearity of $\boldsymbol{I}_{a}$. Thus, $\boldsymbol{K}\left(\boldsymbol{I}_{a}[\mathbf{v}]\right)=0$.

Furthermore, evaluating $\{\nabla \cdot \nabla\}$ and using (2.2) we now conclude that every $\nabla \in \mathcal{C}$ is in fact an Einstein connection.

The most important further step consists in showing, in Section 25, that

the set (2.12) of Einstein connections contains all real-analytic curves $[0, \delta) \ni t \mapsto \mathrm{D}+\mathrm{S}(t)$ of weakly-Einstein connections with $\mathrm{S}(0)=0$.

Assertion (2.13) easily implies the crucial part of our main result (Theorem 22.2):

the family (2.12) of Einstein connections contains all weakly-Einstein connections sufficiently close to the standard connection D.

For $\mathfrak{g}=\mathfrak{s u}(n),(2.14)$ states that a positive-definite multiple of the Killing form is isolated among suitably normalized left-invariant Riemannian Einstein metrics on $\mathrm{SU}(n)$.

The mechanism which reduces proving the inclusion in (2.14) to establishing the analogous claim (2.13) for real-analytic curves is a version of Milnor's curveselection lemma [14, p. 25], stated below as Corollary 4.2, and applicable in our case since both $\mathcal{C}$ and $\mathcal{W}$ are algebraic sets.

The rest of this section is devoted to summarizing the proof of (2.13) given in Section 25. The argument is based on the fundamental formula (2.7) along with the following fact (Lemma 20.1(ii)): suppose that a vector-valued polynomial function $\boldsymbol{P}$ of (2.8) is negligible and (2.8) are themselves $C^{\infty}$ functions of a variable $t \in[0, \delta)$, with $\delta \in(0, \infty)$, which turns $\boldsymbol{P}$ into a function of $t$, and $k \geq 1$ is an integer. In such a case, due to negligibility of $\boldsymbol{P}$,

$$
\text { if } a(0)=0, \xi(0)=h(0)=0 \text {, and } j^{k-1}[b]=0 \text {, then } j^{k}[\boldsymbol{P}]=0 \text {. }
$$

Here $\mathrm{j}^{k}[\ldots]$ denotes the $k$-jet of ... at $t=0$. 
Fixing $t \mapsto \mathrm{S}(t)$ in (2.13), we now realize $a, b, \xi, f, h, x, y, z, p, q, r, \mathbf{v}$ and $\sigma$ as real-analytic functions of $t \in[0, \delta)$. First, a natural surjective operator $\mathcal{S} \rightarrow \mathfrak{g}$ (see Remark 17.4), applied to $\mathrm{S}=\mathrm{S}(t)$, yields $a=a(t) \in \mathfrak{g}$. To define $\sigma(t)$ and $\xi(t)$ we use (2.3) for $\mathrm{S}=\mathrm{S}(t)$ and the relation $\operatorname{tr} a^{2}=n \varepsilon^{-2} \xi$ in (2.9).

Our polynomial mapping $\boldsymbol{J}: \mathbb{F}^{9} \rightarrow \mathbb{F}^{9}$ takes values in $\mathbb{F}^{8}$, as its first component is 0 , while $\operatorname{rank} \mathrm{d} \boldsymbol{J}_{\mathbf{u}}=8$ at our point $\mathbf{u}$ with $\boldsymbol{J}(\mathbf{u})=\mathbf{0}$. Consequently (cf. Lemma 19.1(b)), a neighborhood of $\mathbf{u}$ in $\boldsymbol{J}^{-1}(0)$ forms the graph of an $\mathbb{F}^{8}$-valued $\mathbb{F}$-analytic function $\xi \mapsto(f, h, x, y, z, p, q, r)$ of $\xi$ that varies near 0 in $\mathbb{F}$, with $\mathrm{d} h / \mathrm{d} \xi \neq 0$ at $\xi=0$. Since $\xi$ already is a function of $t \in[0, \delta)$, so are now $f, h, x, y, z, p, q, r$ (with smaller $\delta$ ), $\mathbf{v}=(\xi, f, h, x, y, z, p, q, r)$, and $b$, cf. (2.9).

Next, while proving Lemma 25.1, we establish the following claim:

for all $t \in[0, \delta)$ one has $(\mathrm{S}(t), \sigma(t))=\boldsymbol{I}_{a(t)}[\mathbf{v}(t)]$ and $b(t)=0$.

This is done by using induction on $k$ to show equality between $k$-jets $j^{k}[\ldots]$ of both sides at $t=0$, for all $k \geq 0$. In the induction step, assuming that $j^{k-1}[b]=0$, we use (2.15), with $\boldsymbol{P}$ equal to the left-hand side of (2.7) (for $a=a(t)$ and $\mathbf{v}=\mathbf{v}(t))$ to conclude that $j^{k}[\boldsymbol{P}]=0$. However, our choice of $\mathbf{v}(t)$ guarantees that $\boldsymbol{J}(\mathbf{v}(t))=\mathbf{0}$. Thus, by linearity of $\boldsymbol{I}_{a},(2.7)$ with $\mathbf{j}^{k}[\boldsymbol{P}]=0$ amounts to

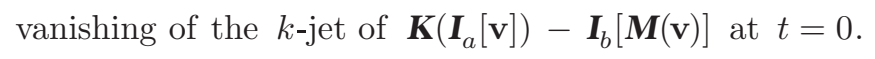

(From now on we write $a, \mathbf{v}$ for $a(t), \mathbf{v}(t)$, etc.) Our quadratic mapping $\boldsymbol{K}$ is the sum of homogeneous components $\boldsymbol{L}$ and $\boldsymbol{K}-\boldsymbol{L}$ of degrees 1 and 2 , cf. Remark 11.3. As $(\mathrm{S}, \sigma)=0$ at $t=0$, the $k$-jet $j^{k}[(\boldsymbol{K}-\boldsymbol{L})(\mathrm{S}, \sigma)]$ depends only on $\mathrm{j}^{k-1}[(\mathrm{~S}, \sigma)]=\mathrm{j}^{k-1}\left[\boldsymbol{I}_{a}[\mathbf{v}]\right]$, the last equality being the remaining part of the inductive-step assumption in our ongoing proof of (2.16). Recalling that our hypothesis about $t \mapsto \mathrm{S}(t)$ in (2.13) reads $\mathbf{K}(\mathrm{S}, \sigma)=(0,0)$ for all $t$ (see (2.4)), we thus have $\mathrm{j}^{k}[\boldsymbol{L}(\mathrm{S}, \sigma)]=\mathrm{j}^{k}[(\boldsymbol{L}-\boldsymbol{K})(\mathrm{S}, \sigma)]=\mathrm{j}^{k}\left[(\boldsymbol{L}-\boldsymbol{K})\left(\boldsymbol{I}_{a}[\mathbf{v}]\right)\right]$ and so, by $(2.17)$,

$$
\mathrm{j}^{k}\left[\boldsymbol{L}\left((\mathrm{S}, \sigma)-\boldsymbol{I}_{a}[\mathbf{v}]\right)\right]=-\mathrm{j}^{k}\left[\boldsymbol{I}_{b}[\boldsymbol{M}(\mathbf{v})]\right] .
$$

Due to the fact that $\boldsymbol{M}$ takes values in a specific two-dimensional vector subspace of $\mathbb{F}^{9}$ (cf. Remark 21.2), one has $\boldsymbol{I}_{b}[\boldsymbol{M}(\mathbf{v})] \in \operatorname{Ker} \boldsymbol{L}$ for all $b \in \mathfrak{g}$ and $\mathbf{v} \in \mathbb{F}^{9}$. However (as a consequence of Remark 11.3 and (12.5.b)), the image of $\boldsymbol{L}$ intersects Ker $\boldsymbol{L}$ trivially, that is, $\boldsymbol{L}$ restricted to its own image must be injective. Thus, both sides in (2.18) must vanish. Now, as $\boldsymbol{M}(\mathbf{u}) \neq \mathbf{0}$, the equality $\mathbf{j}^{k}\left[\mathbf{I}_{b}[\boldsymbol{M}(\mathbf{v})]\right]=(0,0)$ easily implies that $j^{k}[b]=0$. Similarly, since $(S, \sigma)-\boldsymbol{I}_{a}[\mathbf{v}]$ lies in the image of $\boldsymbol{L}$ (see Remark 25.2), the injectivity property just mentioned, combined with vanishing of the left-hand side in (2.18), yields $j^{k}[(S, \sigma)]=j^{k}\left[\mathbf{I}_{a}[\mathbf{v}]\right]$, completing the inductive step and, consequently, proving (2.16).

The induction argument, used above to show that $b(t)=0$ in (2.16), would obviously remain valid if the quantities involved, instead of being real-analytic functions of $t$, were just formal power series in the variable $t$. The subsequent conclusion that $a^{2}=0$ for $a=a(t)$ and every $t$, outlined in the next paragraph 
is, however, less straightforward in this regard, as it relies on certain rationality and nonzero-derivative properties of $h$ and $\xi$.

Specifically, vanishing of $b=b(t)$ in (2.16) gives, by (2.9), $a^{2}=\varepsilon^{-1} h a+$ $\varepsilon^{-2} \xi$ Id for $a=a(t)$ which, unless $a=0$, easily implies that $h^{2}$ is a rational multiple of $\xi$ (see Lemma 24.1). Since $h$ is an $\mathbb{F}$-analytic function of $\xi$ (cf. the lines preceding (2.16)), with $\mathrm{d} h / \mathrm{d} \xi \neq 0=h$ at $\xi=0$, while $\xi$ is a real-analytic function of $t$, the rationality conclusion just stated shows that $\xi(t)=h(t)=0$ identically in $t$. (See the end of Section 25.) Thus, $a=a(t)$ satisfies the condition $a^{2}=0$, while, as $\mathbf{v}=(\xi, f, h, x, y, z, p, q, r)$ is a function of $\xi$ (Lemma 19.1(b)), one also has $\mathbf{v}(t)=\mathbf{u}$ for all $t$. By (2.16), this completes the proof of (2.13).

\section{Linear-algebra preliminaries}

In this section, vector spaces are always finite-dimensional. An inner-product space over $\mathbb{F}=\mathbb{R}$ or $\mathbb{F}=\mathbb{C}$ is a vector space over $\mathbb{F}$ with a fixed $\mathbb{F}$-valued nondegenerate symmetric $\mathbb{F}$-bilinear form. When $\mathbb{F}=\mathbb{R}$, inner-product spaces are what one usually calls pseudo-Euclidean spaces. Note that, except in Section 27, complex inner products are not assumed sesquilinear.

For endomorphisms $\mathrm{A}, \tilde{\mathrm{A}}$ of a vector space $\mathcal{V}$ over $\mathbb{F}=\mathbb{R}$ or $\mathbb{F}=\mathbb{C}$, let

$$
(\mathrm{A}, \tilde{\mathrm{A}})=\operatorname{tr} \mathrm{A} \tilde{\mathrm{A}} \text {. }
$$

Clearly, (, ) turns the endomorphism space End $\mathcal{V}$ into an inner-product space. For endomorphisms $A$ of a complex vector space, with $\operatorname{tr}^{\mathbb{F}}$ denoting the $\mathbb{F}$-trace,

$$
\operatorname{tr}^{\mathbb{R}} \mathrm{A}=2 \operatorname{Re} \operatorname{tr}^{\mathbb{C}} \mathrm{A} .
$$

Treating a complex vector space $\mathcal{V}$ as real and writing $(,)^{\mathbb{F}}$ rather than $($,$) to$ indicate the choice of the scalar field, we have, in $\operatorname{End}_{\mathbb{C}} \mathcal{V}$, by (3.1) and (3.2),

$$
(,)^{\mathbb{R}}=2 \operatorname{Re}(,)^{\mathbb{C}} .
$$

Let us define the adjoint $\Delta^{*}$ of an operator $\Delta$ between inner-product spaces in the usual way, refer to an endomorphism $\Delta$ of such a space $\mathcal{S}$ as self-adjoint if $\Delta^{*}=\Delta$, and call a subspace $\mathcal{S}^{\prime}$ of $\mathcal{S}$ nondegenerate if so is the restriction of the inner product to $\mathcal{S}^{\prime}$. For a self-adjoint endomorphism $\Delta$ of an inner-product space $\mathcal{S}$, with [ [ $]^{\perp}$ denotung the orthogonal complement,

a) the image $\Delta(\mathcal{S})$ coincides with $[\operatorname{Ker} \Delta]^{\perp}$, so that

b) $\mathcal{S}=[\Delta(\mathcal{S})] \oplus \operatorname{Ker} \Delta$ if $\operatorname{Ker} \Delta$ is nondegenerate.

Given subspaces $\mathcal{S}, \mathcal{S}^{\prime}$ of an inner-product space $\mathcal{V}$,

$\mathcal{S}$ is nondegenerate if $\mathcal{V}=\mathcal{S} \oplus \mathcal{S}^{\prime}$ and $\mathcal{S}, \mathcal{S}^{\prime}$ are orthogonal to each other, (3.5) since vectors in $\mathcal{S}$, orthogonal to $\mathcal{S}$, must be orthogonal to all of $\mathcal{V}$. 
Let $\Phi$ be an endomorphism of an inner-product space $\mathcal{S}$. Then $\Phi^{*}=\Phi$ if $\Phi$ is diagonalizable with mutually orthogonal eigenspaces; $\Phi$ has nondegenerate eigenspaces if $\Phi^{*}=\Phi$ and $\Phi$ is diagonalizable.

Cf. (3.5). Obviously, given an endomorphism $\Delta$ of a vector space $\mathcal{S}$,

if $\mathcal{S}=[\Delta(\mathcal{S})] \oplus \operatorname{Ker} \Delta$, then $\Delta: \Delta(\mathcal{S}) \rightarrow \Delta(\mathcal{S})$ is an isomorphism.

Let there be given vector spaces $\mathcal{S}, \mathcal{T}, \mathcal{V}$, an integer $k \geq 1$, and a bilinear mapping $B: \mathcal{S} \times \mathcal{T} \rightarrow \mathcal{V}$, along with $C^{\infty}$ curves $\mathrm{S}$ in $\mathcal{S}$ and $\sigma$ in $\mathcal{T}$, both parametrized by $t \in[0, \delta)$, where $\delta \in(0, \infty)$. If $\mathrm{S}(0)=0$ and $\sigma(0)=0$, then the Leibniz rule clearly implies that, whenever $k \geq 1$,

$$
\mathrm{j}^{k}[B(\mathrm{~S}, \sigma)] \text { depends only on } \mathrm{j}^{k-1}[\mathrm{~S}] \text { and } \mathrm{j}^{k-1}[\sigma] \text {. }
$$

Here and below we use the convention that, for such curves,

$$
j^{k}[\ldots] \text { denotes the } k \text {-jet of } \ldots \text { at } t=0 \text {. }
$$

Remark 3.1. Due to invariance of the trace functional, an endomorphism $\tilde{\mathrm{A}}$ of a vector space such that $J \tilde{A} J^{-1}=\omega \tilde{A}$ for some linear automorphism $J$ and some scalar $\omega \neq 1$ is necessarily traceless. When $\omega=-1$ and $J$ is the multiplication by $\mathrm{i}$ in the underlying real space of a complex vector space, this shows that the real trace of a $\mathbb{C}$-antilinear endomorphism always equals 0.

Remark 3.2. Denoting by $1, \mathrm{i}, \mathrm{j}, \mathrm{k}$ the standard $\mathbb{R}$-basis of the quaternion field $\mathbb{H}$, we identify the real subspace of $\mathbb{H}$ spanned by 1 and i with $\mathbb{C}$, which turns any given vector space $\mathcal{V}$ over $\mathbb{H}$ into a complex vector space. Then $\operatorname{tr}^{\mathbb{C}} \mathrm{A} \in \mathbb{R}$ for all $\mathbb{H}$-linear endomorphisms $\mathrm{A}$ of $\mathcal{V}$, as one sees applying Remark 3.1 and (3.2) to $\tilde{\mathrm{A}}=\mathrm{iA}$, with $\omega=-1$ and $\mathrm{J}$ acting via the $\mathbb{H}$-multiplication by $\mathrm{j}$.

\section{Milnor's curve-selection lemma}

Throughout this section, all vector spaces are finite-dimensional and real.

A subset of a vector space $\mathcal{S}$ is called algebraic if it equals $F^{-1}(0)$ for some polynomial mapping $F: \mathcal{S} \rightarrow \mathcal{V}$ into a vector space $\mathcal{V}$. By a semi-algebraic set in $\mathcal{S}$ one means the intersection of an algebraic set with $\bigcap_{j=1}^{k} f_{j}^{-1}((0, \infty))$, where $k \geq 1$ and $f_{1}, \ldots, f_{k}$ are polynomial functions $\mathcal{S} \rightarrow \mathbb{R}$. The intersection of two semi-algebraic sets in $\mathcal{S}$ is semi-algebraic, while complements of algebraic subsets of $\mathcal{S}$ constitute finite unions of semi-algebraic sets. Thus, whenever $\mathcal{Z} \subset \mathcal{S}$ and $\mathcal{L} \subset \mathcal{S}$ are algebraic, one easily sees that

$$
\mathcal{Z} \backslash \mathcal{L} \text { is a finite union of semi-algebraic sets in } \mathcal{S} .
$$

The following result of Milnor [14, p. 25], known as the curve-selection lemma, generalizes the earlier versions due to Bruhat and Cartan [5, Theorem 1], and Wallace [18, Lemma 18.3]. Further details can be found in [15, p. 402]. 
Theorem 4.1. Suppose that 0 lies in the closure of a semi-algebraic subset $\mathcal{A}$ of a vector space $\mathcal{S}$. Then there exists a real-analytic curve $[0, \delta) \ni t \mapsto \mathrm{S}(t) \in \mathcal{S}$, with $\delta \in(0, \infty)$, such that $\mathrm{S}(0)=0$ and $\mathrm{S}(t) \in \mathcal{A}$ for all $t \in(0, \delta)$.

Proof. See [14, p. 25].

We will need this immediate consequence of Theorem 4.1:

Corollary 4.2. Let $\mathcal{Z}$ and $\mathcal{L}$ be algebraic sets in a vector space $\mathcal{S}$. If $\mathcal{L}$ contains every real-analytic curve $[0, \delta) \ni t \mapsto \mathrm{S}(t) \in \mathcal{S}$ with $\mathrm{S}(0)=0$, lying entirely in $\mathcal{Z}$, then $\mathcal{L}$ also contains all points of $\mathcal{Z}$ sufficiently close to 0 .

Proof. Otherwise, an obvious contradiction would arise from Theorem 4.1 applied to $\mathcal{A}$ which is one of the semi-algebraic sets mentioned in (4.1).

\section{The curvature and Ricci tensors}

We denote by $R=R^{\nabla}$ the curvature tensor of a torsion-free $C^{\infty}$ connection $\nabla$ on a manifold, using the sign convention

$$
R(v, w) u=\nabla_{w} \nabla_{v} u-\nabla_{v} \nabla_{w} u+\nabla_{[v, w]} u \text {. for } C^{\infty} \text { vector fields } u, v, w .
$$

The Ricci tensor $\rho=\rho^{\nabla}$ has the components $R_{j k}=R_{j s k}{ }^{s}$. Repeated indices, here and below, are to be summed over.

Any torsion-free $C^{\infty}$ connection $\nabla$ satisfies the Bochner identity

$$
\rho(\cdot, w)=\operatorname{div} \nabla w-\mathrm{d}(\operatorname{div} w) \quad \text { for every } C^{\infty} \text { vector field } w,
$$

cf. [3, pp. 57, 74, 166], Here $\operatorname{div} w=\operatorname{tr} \nabla w$, with $\nabla w$ treated as the endomorphism of the tangent bundle acting on vector fields $v$ by $v \mapsto \nabla_{v} w$.

The coordinate form of (5.2), $R_{j k} w^{k}=w^{k}{ }_{, j k}-w^{k}{ }_{, k j}$, arises by contraction in $l=k$ from the Ricci identity $w^{l}{ }_{, j k}-w^{l}{ }_{, k j}=R_{j k s}{ }^{l} w^{s}$, which in turn is the coordinate form of (5.1). Evaluated on a $C^{\infty}$ vector field $v,(5.2)$ gives

$$
\rho(v, w)=\operatorname{div}\left[\nabla_{v} w-(\operatorname{div} w) v\right]+(\operatorname{div} v) \operatorname{div} w-(\nabla v, \nabla w)
$$

via differentiation by parts. For the meaning of $(\nabla v, \nabla w)$, see (3.1).

By an Einstein metric on a manifold we mean any $C^{\infty}$ pseudo-Riemannian metric $\gamma$ with $\rho=\kappa \gamma$ for the Ricci tensor $\rho$ of $\gamma$ (that is, the Ricci tensor of the Levi-Civita connection of $\gamma$ ) and some $\kappa \in \mathbb{R}$ (the Einstein constant of $\gamma$ ).

One calls a torsion-free $C^{\infty}$ connection $\nabla$ on an $m$-dimensional manifold equiaffine if the connection induced by $\nabla$ in the $m$ th exterior power of the tangent bundle is flat, that is, if the manifold locally admits $\nabla$-parallel volume forms.

Equiaffinity of $\nabla$ is equivalent to symmetry of its Ricci tensor $\rho^{\nabla}$. This well-known fact [17, III 4,5 and (5.8), pp. 144-145] is not used in our argument.

Remark 5.1. The whole discussion in this section remains valid for holomorphic connections and holomorphic metrics on complex manifolds [12, pp. 210-211]. 
Remark 5.2. Recall that the Levi-Civita connection of a $C^{\infty}$ or holomorphic metric is the unique torsion-free connection making the metric parallel [12, p. 211].

\section{Left-invariant connections on Lie groups}

Let $G$ be a real or complex Lie group. Its Lie algebra $\mathfrak{g}$, over $\mathbb{F}=\mathbb{R}$ or $\mathbb{F}=\mathbb{C}$, is always identified with the space of left-invariant vector fields on G. In addition, left-invariant connections on $G$ are assumed holomorphic when $\mathbb{F}=\mathbb{C}$. Similarly, left-invariant metrics on $\mathrm{G}$ are by definition pseudo-Riemannian if $\mathbb{F}=\mathbb{R}$, and holomorphic (in the sense of being $\mathbb{C}$-bilinear, symmetric and nondegenerate) if $\mathbb{F}=\mathbb{C}$. Cf. also Remark 5.1.

Left-invariant connections $\nabla$ on $\mathrm{G}$, and left-invariant symmetric 2-tensor fields $\tau$ on $\mathrm{G}$ (including metrics) are always treated as elements of $\mathcal{Y}=\left[\mathfrak{g}^{*}\right]^{\otimes 2} \otimes \mathfrak{g}$ (or, $\mathcal{T}=\left[\mathfrak{g}^{*}\right]^{\odot 2}$ ), that is, $\mathbb{F}$-bilinear operations $\nabla: \mathfrak{g} \times \mathfrak{g} \rightarrow \mathfrak{g}$ (or, respectively, symmetric $\mathbb{F}$-bilinear forms $\tau: \mathfrak{g} \times \mathfrak{g} \rightarrow \mathbb{F}$ ). For $v, w \in \mathfrak{g}$, we then use the traditional symbol $\nabla_{v} w$, rather than $\nabla(v, w)$, and denote by $\nabla_{v}$ (or, by $\nabla w$ ) the $\mathbb{F}$-linear endomorphism of $\mathfrak{g}$ sending $w$ (or, respectively, $v$ ) to $\nabla_{v} w$.

A left-invariant torsion-free connection $\nabla$ on $\mathrm{G}$ will be called unimodular if some/any left-invariant real/complex volume form on $\mathrm{G}$ is $\nabla$-parallel.

Unimodularity of $\nabla$ clearly implies its equiaffinity (see the end of Section 5 ).

Lemma 6.1. Let $\nabla$ be a left-invariant torsion-free connection on a real/complex Lie group $\mathrm{G}$ with the Lie algebra $\mathfrak{g}$ over $\mathbb{F}=\mathbb{R}$ or $\mathbb{F}=\mathbb{C}$.

(a) $\nabla$ is unimodular if and only if $\operatorname{tr} \nabla_{v}=0$ for all $v \in \mathfrak{g}$.

(b) $\nabla$ is unimodular whenever there exists a nondegenerate left-invariant $\nabla$-parallel twice-covariant symmetric tensor field on $\mathrm{G}$, that is, whenever $\nabla$ is the Levi-Civita connection of some left-invariant metric.

(c) If $\mathrm{G}$ is unimodular, then unimodularity of $\nabla$ is equivalent to tracelessness of $\nabla w$ for every $w \in \mathfrak{g}$, that is, to the condition $\operatorname{div} w=0$ for all $w \in \mathfrak{g}$.

(d) If both $\mathrm{G}$ and $\nabla$ are unimodular, then the Ricci tensor $\rho=\rho^{\nabla}$ is given by $\rho(v, w)=-(\nabla v, \nabla w)$ for $v, w \in \mathfrak{g}, c f$. (3.1). In particular, $\rho$ is symmetric.

(e) If $\mathrm{G}$ is unimodular and the tensor $\rho$ given by $\rho(v, w)=-(\nabla v, \nabla w)$ is nondegenerate and $\nabla$-parallel, then $\nabla$ is unimodular and $\rho$ is its Ricci tensor.

Proof. Let $m=\operatorname{dim}_{\mathbb{F}} \mathfrak{g}$. For the connection $\bar{\nabla}$ induced by $\nabla$ in the $m$ th exterior power, over $\mathbb{F}$, of the tangent bundle of $\mathrm{G}$, and any basis $e_{1}, \ldots, e_{m}$ of $\mathfrak{g}$, one clearly has $\bar{\nabla}_{v}\left(e_{1} \wedge \ldots \wedge e_{m}\right)=\left(\operatorname{tr} \nabla_{v}\right) e_{1} \wedge \ldots \wedge e_{m}$, which proves (a). To obtain (b), note that, for an orthonormal basis $e_{1}, \ldots, e_{m}$, the $m$-vector $e_{1} \wedge \ldots \wedge e_{m}$ is parallel due to its being uniquely determined, up to a sign, by the metric. Assertion (c) follows in turn from (a), since unimodularity of G amounts to tracelessness of $\operatorname{Ad} v$ for all $v, w \in \mathfrak{g}$, and $\operatorname{Ad} v=\nabla_{v}-\nabla v$ as $\nabla$ is torsion-free, while (d) is obvious from (5.3), along with (c) applied to the left-invariant vector fields $w$ and $\nabla_{v} w-(\operatorname{div} w) v$. Finally, (e) is a consequence of (b), for the metric $\rho$, and (d). 
Remark 6.2. By assigning, to any left-invariant Einstein metric with Einstein constant 1 on a Lie group G, its Levi-Civita connection, we obtain a bijective mapping between the set of such metrics and the set of all left-invariant torsion-free connections $\nabla$ on $\mathrm{G}$ having symmetric, nondegenerate, $\nabla$-parallel Ricci tensors. The inverse mapping sends $\nabla$ to its Ricci tensor.

This is a trivial consequence of Remark 5.2.

Remark 6.3. We will use the fact that every semisimple Lie group is unimodular.

\section{Some operations involving connections and 2-tensors}

In this section, $\mathfrak{g}$ is a fixed semisimple Lie algebra over $\mathbb{F}=\mathbb{R}$ or $\mathbb{F}=\mathbb{C}$.

As in the second paragraph of Section 6 , we identify elements $\nabla$ of the space $\mathcal{Y}=\left[\mathfrak{g}^{*}\right]^{\otimes 2} \otimes \mathfrak{g}$ with left-invariant connections on a Lie group $\mathrm{G}$ having the Lie algebra $\mathfrak{g}$, writing $\nabla_{v} w, \nabla_{v}$ and $\nabla w$ instead of $\nabla(v, w), \nabla(v, \cdot)$ and $\nabla(\cdot, w)$, for $v, w \in \mathfrak{g}$, so that $\nabla_{v}, \nabla w: \mathfrak{g} \rightarrow \mathfrak{g}$. We will repeatedly refer to the vector spaces

$$
\mathcal{T}=\left[\mathfrak{g}^{*}\right]^{\odot 2}, \quad \mathcal{S}=\left[\mathfrak{g}^{*}\right]^{\odot 2} \otimes \mathfrak{g} \subset \mathcal{Y}=\left[\mathfrak{g}^{*}\right]^{\otimes 2} \otimes \mathfrak{g} .
$$

Note that $\mathcal{T} \subset\left[\mathfrak{g}^{*}\right]^{\otimes 2}$ and $\mathcal{S} \subset\left[\mathfrak{g}^{*}\right]^{\otimes 2} \otimes \mathfrak{g}$ consist of all $\mathbb{F}$-valued (or, respectively, $\mathfrak{g}$-valued) symmetric $\mathbb{F}$-bilinear mappings defined on $\mathfrak{g} \times \mathfrak{g}$.

Elements of $\mathcal{T}$ may, again, be thought of as twice-covariant symmetric tensor fields on $\mathrm{G}$, invariant under left translations. The symbols $\beta$ and $\langle$,$\rangle both stand$ for the $\mathbb{F}$-bilinear Killing form of $\mathfrak{g}$. Thus, $\beta \in \mathcal{T}$ and, with $($,$) as in (3.1),$

$$
\beta(v, w)=\langle v, w\rangle=(\operatorname{Ad} v, \operatorname{Ad} w) \quad \text { whenever } v, w \in \mathfrak{g} .
$$

The $\mathbb{F}$-bilinear inner product $\beta=\langle$,$\rangle in \mathfrak{g}$ leads to an isomorphic identification between bilinear forms $\sigma$ on $\mathfrak{g}$ and $\mathbb{F}$-linear endomorphisms $\Sigma$ of $\mathfrak{g}$, with

$$
\sigma(v, w)=\langle\Sigma v, w\rangle \quad \text { for any } \quad v, w \in \mathfrak{g} .
$$

Left-invariant connections on $\mathrm{G}$ which are torsion-free form an affine subspace of $\left[\mathfrak{g}^{*}\right]^{\otimes 2} \otimes \mathfrak{g}$, namely, the coset $\mathrm{D}+\mathcal{S}$, where $\mathrm{D}$ is the standard connection, with

$$
2 \mathrm{D}_{v} w=[v, w], \quad \text { so that } \mathrm{D}_{v}=-\mathrm{D} v=\operatorname{Ad} v / 2 \text {. }
$$

We define the contraction operator $\mathbf{c}: \mathcal{Y}=\left[\mathfrak{g}^{*}\right]^{\otimes 2} \otimes \mathfrak{g} \rightarrow \mathfrak{g}^{*}$ by $(\mathbf{c} \nabla) w=\operatorname{tr} \nabla w$. In view of Lemma 6.1(c) and Remark 6.3, for any $\nabla=\mathrm{D}+\mathrm{S}$, where $\mathrm{S} \in \mathcal{S}$,

$$
\mathrm{D}+\mathrm{S} \text { is unimodular if and only if } \mathbf{c S}=0 \text {. }
$$

Tensor-calculus arguments, when needed, will use components relative to a fixed basis $e_{1}, \ldots, e_{m}$ of $\mathfrak{g}$, with $m=\operatorname{dim}_{\mathbb{F}} \mathfrak{g}$. Vectors $v \in \mathfrak{g}$, bilinear forms $\sigma$ on $\mathfrak{g}$, connections $\nabla \in \mathcal{Y}=\left[\mathfrak{g}^{*}\right]^{\otimes 2} \otimes \mathfrak{g}$, and symmetric operations $\mathrm{S} \in \mathcal{S}$ are represented by the components $v^{j}, \sigma_{j k}, \Gamma_{j k}^{l}$ and $S_{j k}^{l}=S_{k j}^{l}$ characterized by $v=v^{j} e_{j}$, $\sigma(v, w)=\sigma_{j k} v^{j} w^{k},\left[\nabla_{v} w\right]^{l}=\Gamma_{j k}^{l} v^{j} w^{k}$ and $\left[\mathrm{S}_{v} w\right]^{l}=S_{j k}^{l} v^{j} w^{k}$. Note the traditional symbol $\Gamma_{j k}^{l}$, rather than $\nabla_{j k}^{l}$. For the components $D_{j k}^{l}$ of $\mathrm{D}$ we have $2 D_{j k}^{l}=C_{j k}^{l}$, 
where $C_{j k}^{l}$ are the structure constants of $\mathfrak{g}$, with $[v, w]^{l}=C_{j k} v^{j} w^{k}$ or, equivalently, $\left[e_{j}, e_{k}\right]=C_{j k}{ }^{l} e_{l}$. The reciprocal tensor of the Killing form $\beta$ has the components $\beta^{j k}$, forming the inverse matrix of $\left[\beta_{j k}\right]$. We will raise and lower indices with the aid of $\beta^{j k}$ and $\beta_{j k}$, so that $C^{j}{ }_{k}^{l}=\beta^{j s} C_{s k}{ }^{l}, C_{j k l}=C_{j k}{ }^{s} \beta_{s l}$, etc. If an endomorphism $\Sigma$ of $\mathfrak{g}$ corresponds to $\sigma$ via (7.3), its components will be written as $\sigma_{j}^{k}$, and then $[\Sigma v]^{k}=\sigma_{j}^{k} v^{j}$, while $\sigma_{j}^{k}=\sigma_{j s} \beta^{s k}$. Thus, for the contraction operator c appearing in (7.5),

$$
[\mathbf{c} \nabla]_{j}=\Gamma_{k j}^{k} .
$$

Since the Killing form $\beta$ is bi-invariant (that is, Ad-invariant), one has

$$
(\operatorname{Ad} v, \Sigma)=0 \text { whenever } v \in \mathfrak{g} \text { and } \Sigma: \mathfrak{g} \rightarrow \mathfrak{g} \text { is } \beta \text {-self-adjoint, }
$$

due to skew-adjointness of $\operatorname{Ad} v$, with $($,$) as in (3.1). For the same reason,$

$$
C_{j k l} \text { is totally skew-symmetric in } j, k, l \text {, }
$$

which amounts to skew-symmetry of $\mathfrak{g} \times \mathfrak{g} \times \mathfrak{g} \ni(v, w, u) \mapsto \beta([v, w], u) \in \mathbb{R}$.

\section{Further operations}

We continue using the same assumptions and notations as in Section 7.

The Killing form $\beta=\langle$,$\rangle , being an \mathbb{F}$-bilinear inner product in $\mathfrak{g}$, naturally induces $\mathbb{F}$-bilinear inner products, also denoted by $\langle$,$\rangle , in the spaces \left[\mathfrak{g}^{*}\right]^{\otimes 2}$ and $\mathcal{Y}=\left[\mathfrak{g}^{*}\right]^{\otimes 2} \otimes \mathfrak{g}$. (Their nondegeneracy is obvious, since a $\beta$-orthonormal basis of $\mathfrak{g}$ gives rise to $\langle$,$\rangle -orthonormal bases in the other two spaces.) Explicitly,$

$$
\text { i) }\langle\sigma, \tau\rangle=\beta^{j p} \beta^{k q} \sigma_{j k} \tau_{p q}, \quad \text { ii) }\langle\nabla, \tilde{\nabla}\rangle=\beta^{j p} \beta^{k q} \beta_{l r} \Gamma_{j k}^{l} \tilde{\Gamma}_{p q}^{r} .
$$

for any $\sigma, \tau \in\left[\mathfrak{g}^{*}\right]^{\otimes 2}$ and $\nabla, \tilde{\nabla} \in \mathcal{Y}=\left[\mathfrak{g}^{*}\right]^{\otimes 2} \otimes \mathfrak{g}$. Furthermore,

$$
(\mathcal{S},\langle,\rangle) \text { and }(\mathcal{T},\langle,\rangle) \text { are inner-product spaces. }
$$

In other words, $\mathcal{S} \subset \mathcal{Y}$ and $\mathcal{T} \subset\left[\mathfrak{g}^{*}\right]^{\otimes 2}$ appearing in (7.1) are nondegenerate subspaces, which one easily sees applying (3.5) to our $\mathcal{S}$ and $\mathcal{S}^{\prime}=\left[\mathfrak{g}^{*}\right]^{\wedge 2} \otimes \mathfrak{g}$, with $\mathcal{V}=\mathcal{Y}$, or, respectively, to $\mathcal{T}$ instead of $\mathcal{S}$, with $\mathcal{S}^{\prime}=\left[\mathfrak{g}^{*}\right]^{\wedge 2}$ and $\mathcal{V}=\left[\mathfrak{g}^{*}\right]^{\otimes 2}$.

For $\nabla \in \mathcal{Y}=\left[\mathfrak{g}^{*}\right]^{\otimes 2} \otimes \mathfrak{g}$, the $\nabla$-gradient of any $\sigma \in \mathcal{T}$ is

$$
\mathrm{S}=\nabla \sigma \in \mathcal{S} \text {, characterized by }\left\langle\mathrm{S}_{v} w, u\right\rangle=-\sigma\left(\nabla_{u} v, w\right)-\sigma\left(v, \nabla_{u} w\right) .
$$

(Note that $\nabla \sigma$ differs only slightly from the $\nabla$-covariant derivative of $\sigma$, from which it arises via index-raising applied to the direction of differentiation.) Given such $\nabla$ and $\sigma$, we also define

$$
\tilde{\nabla}=\sigma \nabla \in \mathcal{S} \text {, by declaring } \tilde{\nabla}_{v} w \text { to be } \Sigma\left(\nabla_{v} w\right) \text {, for } \Sigma \text { with }(7.3) .
$$

Equivalently, $\left\langle\tilde{\nabla}_{v} w, u\right\rangle=\sigma\left(\nabla_{v} w, u\right)$ and, in terms of components,

$$
\text { a) } S_{j k}^{l}=-\beta^{l p}\left(\Gamma_{p j}^{r} \sigma_{r k}+\Gamma_{p k}^{r} \sigma_{j r}\right) \text { if } \mathrm{S}=\nabla \sigma, \quad \text { b) } \tilde{\Gamma}_{j k}^{l}=\sigma_{r}^{l} \Gamma_{j k}^{r} \text { if } \tilde{\nabla}=\sigma \nabla \text {. }
$$


For $\mathrm{D}$ as in (7.4), and any $\sigma \in \mathcal{T}$, (8.5.a) with $\mathrm{S}=\mathrm{D} \sigma$ and $\Gamma_{j k}^{l}=C_{j k}^{l} / 2$ gives, by (7.8), $2 S_{r k}^{r}=-C^{l}{ }_{l}^{r} \sigma_{r k}-C^{j}{ }_{k}^{r} \sigma_{j r}=0$. Thus, from (7.6),

$$
\mathbf{c}(\mathrm{D} \sigma)=0 \quad \text { whenever } \quad \sigma \in \mathcal{T} \text {. }
$$

In view of the line following (7.8), the three definitions: (8.3) of $\nabla \sigma$ (applied to $\nabla=\mathrm{D}),(7.4)$ of $\mathrm{D}$, and (8.4) of $\sigma \nabla$ (applied to $\sigma=\tau$ and $\nabla=\mathrm{D} \sigma$ ), imply that for any $\sigma, \tau \in \mathcal{T}$ and $\Sigma$, T related to them as in (7.3), since $\Sigma$ is $\beta$-self-adjoint,

$$
\text { a) }[\mathrm{D} \sigma]_{v} v=[\Sigma v, v], \quad \text { b) }[\tau(\mathrm{D} \sigma)]_{v} v=\mathrm{T}[\Sigma v, v]
$$

The normalized $\beta$-curvature operator $\Omega: \mathcal{T} \rightarrow \mathcal{T}$ is given by

$$
[\Omega \sigma](v, w)=2(\operatorname{Ad} v,(\operatorname{Ad} w) \Sigma) \quad \text { for } v, w \in \mathfrak{g},
$$

with (, ) as in (3.1), any $\sigma \in \mathcal{T}$, and $\Sigma$ corresponding to $\sigma$ via (7.3). We have

$$
\text { i) } \Omega \beta=2 \beta \text {, ii) }[\Omega \sigma]_{j k}=2 C_{j q}{ }^{r} C_{k s}{ }^{q} \sigma_{r}^{s}, \quad \text { iii) } \operatorname{tr} \Omega=-\operatorname{dim}_{\mathbb{F}} \mathfrak{g} \text {. }
$$

In fact, (8.9.i) and (8.9.ii) are obvious from (7.2) and (8.8). To verify (8.9.iii), we extend $\Omega: \mathcal{T} \rightarrow \mathcal{T}$ to $\tilde{\Omega}:\left[\mathfrak{g}^{*}\right]^{\otimes 2} \rightarrow\left[\mathfrak{g}^{*}\right]^{\otimes 2}$ defined by the same formula (8.9.ii), so that $[\tilde{\Omega} \sigma]_{j k}=Z_{j k}^{r s} \sigma_{r s}$ with $Z_{j k}^{r s}=2 C_{j q}{ }^{r} C_{k}{ }^{s q}$. Thus, $\operatorname{tr} \tilde{\Omega}=Z_{r s}^{r s}=0$ (cf. (7.8)). At the same time, $Z_{j k}^{r s}=Z_{k j}^{s r}$, so that $\tilde{\Omega}$ leaves the subspace $\left[\mathfrak{g}^{*}\right]^{\wedge 2}$ invariant, and acts on $\sigma \in\left[\mathfrak{g}^{*}\right]^{\wedge 2}$ via $Z_{j k}^{r s} \sigma_{r s}=\left(Z_{j k}^{r s}-Z_{k j}^{r s}\right) \sigma_{r s} / 2$ which, by the Jacobi identity, gives $Z_{j k}^{r s} \sigma_{r s}=H_{j k}^{r s} \sigma_{r s}$ for $H_{j k}^{r s}=2 C_{k j q} C^{s q r}$. Due to skew-symmetry of $H_{j k}^{r s}$ in both $j, k$ and $r, s$ (see $(7.8)$ ), the trace of the restriction of $\tilde{\Omega}$ to $\left[\mathfrak{g}^{*}\right]^{\wedge 2}$ is obtained as the sum of $H_{r s}^{r s}$ over the pairs $r, s$ with $r<s$. In terms of the ordinary summing convention, this last trace equals $H_{r s}^{r s} / 2=C_{s r q} C^{s q r}=\beta_{s}^{s}=\operatorname{dim}_{\mathbb{F}} \mathfrak{g}$, as $\beta_{j k}=C_{j q}{ }^{p} C_{k p}{ }^{q}$ by (7.2). Since $\operatorname{tr} \tilde{\Omega}=0$, (8.9.iii) follows.

The name used for $\Omega$ reflects the fact that, if one treats the Killing form $\beta$ as a left-invariant pseudo-Riemannian Einstein metric on a Lie group with the Lie algebra $\mathfrak{g}$, then $\Omega$ is, up to a factor, the curvature operator of $\beta$, acting on symmetric 2-tensors [7, Remark 1.4].

\section{Two symmetric pairings}

We continue the discussion of Sections $7-8$. The symbol $\{\cdot\}$ denotes two $\mathcal{T}$-valued symmetric bilinear mappings defined on $\mathcal{Y}$ (and, respectively, on $\mathcal{T}$ ), characterized by the corresponding $\mathcal{T}$-valued homogeneous quadratic functions:

$$
\{\nabla \cdot \nabla\}(v, w)=(\nabla v, \nabla w), \quad\{\sigma \cdot \sigma\}(v, w)=((\operatorname{Ad} v) \Sigma,(\operatorname{Ad} w) \Sigma)
$$

for $\nabla \in \mathcal{Y}, \sigma \in \mathcal{T}$ and $v, w \in \mathfrak{g}$, with $\Sigma$, (, ) as in (7.3) and (3.1). Equivalently,

$$
2\{\nabla \cdot \tilde{\nabla}\}_{j k}=\Gamma_{r j}^{s} \tilde{\Gamma}_{s k}^{r}+\tilde{\Gamma}_{r j}^{s} \Gamma_{s k}^{r}, \quad 2\{\sigma \cdot \tau\}_{j k}=C_{j r}{ }^{p} \sigma_{q}^{r} C_{k s}{ }^{q} \tau_{p}^{s}+C_{j r}{ }^{p} \tau_{q}^{r} C_{k s}{ }^{q} \sigma_{p}^{s}
$$

whenever $\nabla, \tilde{\nabla} \in \mathcal{Y}=\left[\mathfrak{g}^{*}\right]^{\otimes 2} \otimes \mathfrak{g}$ and $\sigma, \tau \in \mathcal{T}$. By (8.8), for $\sigma \in \mathcal{T}$,

$$
2\{\sigma \cdot \beta\}=\Omega \sigma .
$$


Being symmetric, $\{\sigma \cdot \tau\}$ is uniquely characterized by the requirement that

$$
\{\sigma \cdot \tau\}(w, w)=((\operatorname{Ad} w) \Sigma,(\operatorname{Ad} w) \mathrm{T}) \quad \text { for all } w \in \mathfrak{g},
$$

with $\Sigma, \mathrm{T}$ as in (8.7.b). Lemma 6.1(d) and (9.1) imply that a left-invariant torsion-free unimodular connection $\nabla=\mathrm{D}+\mathrm{S}$, with $\mathrm{S} \in \mathcal{S}$, has the Ricci tensor

$$
\rho^{\nabla}=-\{\nabla \cdot \nabla\}
$$

cf. Remark 6.3. Note that, due to (7.2), (7.4), (9.5), (7.8) and (8.7.a),

$$
\text { a) } 4\{\mathrm{D} \cdot \mathrm{D}\}=\beta \text {, that is, } \beta_{j k}=C_{p j}{ }^{q} C_{q k}{ }^{p} \text {, b) } 4 \rho^{\mathrm{D}}=-\beta, \text { c) } \mathrm{D} \beta=0 \text {. }
$$

Finally, for the inner products in (8.2), any $\mathrm{S} \in \mathcal{S}$, and any $\sigma \in \mathcal{T}$,

$$
\text { a) }\langle\mathrm{S}, \mathrm{D} \sigma\rangle=2\langle\{\mathrm{D} \cdot \mathrm{S}\}, \sigma\rangle, \quad \text { b) }\{\mathrm{D} \cdot(\mathrm{S} \beta)\}=\{\mathrm{D} \cdot \mathrm{S}\} .
$$

In fact, since $S_{j k}^{l}=S_{k j}^{l}, \sigma_{j k}=\sigma_{k j}, 2 D_{j k}^{l}=C_{j k}^{l}$, (8.1.ii), (8.5.a) and (7.8) give $\langle\mathrm{S}, \mathrm{D} \sigma\rangle=-\beta^{j p} \beta^{k q} S_{j k}^{l} C_{l p}{ }^{r} \sigma_{r q}=-S_{j k}^{l} C_{l}{ }^{j r} \sigma_{r}^{k}=S_{j k}^{l} C_{l r}{ }^{j} \sigma^{k r}=2\{\mathrm{D} \cdot \mathrm{S}\}_{k r} \sigma^{k r}=$ $2\langle\{\mathrm{D} \cdot \mathrm{S}\}, \sigma\rangle$. For similar reasons, one has $4\{\mathrm{D} \cdot(\mathrm{S} \beta)\}_{j k}=-C^{p}{ }_{j}{ }^{q}\left(S_{p q}^{r} \beta_{r k}+S_{p k}^{r} \beta_{q r}\right)-$ $C_{k}^{p}{ }^{q}\left(S_{p q}^{r} \beta_{r j}+S_{p j}^{r} \beta_{q r}\right)$. As a consequence of (7.8), the first and third of the four resulting terms vanish, and the other two add up to $C_{r j}{ }^{p} S_{p k}^{r}+C_{r k}{ }^{p} S_{p j}^{r}=4\{\mathrm{D} \cdot \mathrm{S}\}_{j k}$.

\section{The Christoffel isomorphism}

With the assumptions and notations as in the last three sections, let us introduce the Christoffel isomorphism $\Phi: \mathcal{S} \rightarrow \mathcal{S}$ by requiring that, whenever $v, w, u \in \mathfrak{g}$,

$$
2\left\langle u, \tilde{\mathrm{S}}_{v} w\right\rangle=\left\langle v, \mathrm{~S}_{w} u\right\rangle+\left\langle w, \mathrm{~S}_{v} u\right\rangle-\left\langle u, \mathrm{~S}_{v} w\right\rangle \quad \text { for } \tilde{\mathrm{S}}=\Phi \mathrm{S} .
$$

We give two more descriptions of $\Phi$, one based on applying, to $\nabla=\mathrm{S}$ and $\sigma=\beta$, the definition (8.3) of the $\nabla$-gradient, the other using components and (8.5.a):

$$
\text { i) } 2 \Phi \mathrm{S}=-\mathrm{S} \beta-\mathrm{S}, \quad \text { ii) } 2(\Phi \mathrm{S})_{j k}^{l}=\beta^{l p}\left(S_{p j}^{r} \beta_{r k}+S_{p k}^{r} \beta_{r j}\right)-S_{j k}^{l} \text {. }
$$

For yet another description of $\Phi$, see Remark 10.2. If $\mathrm{S} \in \mathcal{S}$ and $\sigma \in \mathcal{T}$, then

$$
\text { a) } \Phi(\mathrm{D} \sigma)=-\mathrm{D} \sigma, \quad \text { b) } \Phi(\mathrm{S} \sigma)=-\sigma \mathrm{S} \text {, }
$$

as one sees combining the line following (7.8) and (10.1) with (8.7.a) or, respectively, applying the definitions of $\nabla \sigma$ and $\sigma \nabla$ in (8.3) - (8.4) to $\nabla=\mathrm{S}$. Also,

$$
\text { a) } \Phi(\mathrm{S} \beta)=-\mathrm{S}, \quad \text { b) }\{\mathrm{D} \cdot(\Phi \mathrm{S})\}=-\{\mathrm{D} \cdot \mathrm{S}\} \text {. }
$$

In fact, (10.4.a) is a special case of (10.3.b), for $\sigma=\beta$, while (10.2.i) and (9.7.b) give $2\{\mathrm{D} \cdot(\Phi \mathrm{S})\}=-\{\mathrm{D} \cdot(\mathrm{S} \beta)\}-\{\mathrm{D} \cdot \mathrm{S}\}=-2\{\mathrm{D} \cdot \mathrm{S}\}$, proving (10.4.b).

Note that $\Phi$ is actually an isomorphism, as a consequence of (10.4.a).

In the next lemma, $\mathrm{D} \lambda$, or $\mathrm{D} \chi$ or, respectively, $(\mathrm{D} \lambda) \psi$ is given by (8.3) with $\nabla=\mathrm{D}$ and $\sigma=\lambda$, or $\nabla=\mathrm{D}$ and $\sigma=\chi$ or, respectively, $\nabla=\mathrm{D} \lambda$ and $\sigma=\psi$. Similarly, $\psi(\mathrm{D} \lambda)$ is defined as in (8.4) with $\sigma=\psi$ and $\nabla=\mathrm{D} \lambda$.

Lemma 10.1. If $\psi, \lambda, \chi \in \mathcal{T}$, for $\mathcal{T}$ in (7.1), and $\psi(\mathrm{D} \lambda)=\mathrm{D} \chi$, then $(\mathrm{D} \lambda) \psi=\mathrm{D} \chi$. 
Proof. From (10.3) with $\mathrm{S}=\mathrm{D} \lambda$ we get $\Phi[(\mathrm{D} \lambda) \psi-\mathrm{D} \chi]=\mathrm{D} \chi-\psi(\mathrm{D} \lambda)$.

The operation $\mathcal{T} \times \mathcal{T} \ni(\sigma, \tau) \mapsto \sigma \circledast \tau \in \mathcal{T}$ is defined by requiring it to correspond, under the identification in $(7.3)$, to $(\Sigma, \tilde{\Sigma}) \mapsto(\Sigma \tilde{\Sigma}+\tilde{\Sigma} \Sigma) / 2$ (onehalf of the anticommutator of $\beta$-self-adjoint linear endomorphisms of $\mathfrak{g}$ ). Thus, $\beta \circledast \sigma=\sigma$. Any nondegenerate $\gamma \in \mathcal{T}$ has an inverse $\gamma^{-1} \in \mathcal{T}$ with $\gamma \circledast \gamma^{-1}=\beta$.

Every $\sigma \in \mathcal{T}$ satisfies the following relations, in which, once again, $\mathrm{D}, \beta$ and $\Omega$ are the standard connection, Killing form, and normalized $\beta$-curvature operator, with (7.4), (7.2) and (8.8), $\{\cdot\}$ stands for the two operations given by $(9.1)$ or $(9.2)$, while $\circledast$ is defined as in the last paragraph:

a) $(\mathrm{D} \sigma) \beta=\mathrm{D} \sigma$,

b) $8\{\mathrm{D} \cdot(\mathrm{D} \sigma)\}=(\Omega-2 \mathrm{Id}) \sigma$,

c) $4\{(\mathrm{D} \sigma) \cdot(\mathrm{D} \sigma)\}=\{\sigma \cdot \sigma\}+[(\mathrm{Id}-\Omega) \sigma] \circledast \sigma$,

d) $4\{(\mathrm{D}+\mathrm{D} \sigma) \cdot(\mathrm{D}+\mathrm{D} \sigma)\}=\beta-\sigma+\{\sigma \cdot \sigma\}+[(\Omega-\mathrm{Id}) \sigma] \circledast(\beta-\sigma)$.

In fact, $(\mathrm{D} \sigma) \beta=\mathrm{D} \sigma$ since $\Phi[(\mathrm{D} \sigma) \beta]=\Phi(\mathrm{D} \sigma)$ by (10.4.a) (for $\mathrm{S}=\mathrm{D} \sigma$ ) and (10.3.a). Next, $-8\{\mathrm{D} \cdot(\mathrm{D} \sigma)\}_{j k}=C_{p j}{ }^{q}\left(C^{p}{ }_{q r} \sigma_{k}^{r}+C^{p}{ }_{k r} \sigma_{q}^{r}\right)+C_{p k}{ }^{q}\left(C^{p}{ }_{q r} \sigma_{j}^{r}+C^{p}{ }_{j r} \sigma_{q}^{r}\right)$ due to (8.5.a) with $\Gamma_{j k}^{l}=D_{j k}^{l}=C_{j k}^{l} / 2$ and (9.2). In view of (9.6.a) and (7.8), the first and third of the resulting four terms are both equal to $\beta_{j r} \sigma_{k}^{r}=\beta_{k r} \sigma_{j}^{r}=\sigma_{j k}$, and their sum is $2 \sigma_{j k}$. The other two terms are also equal and, by (8.9.ii), add up to $-[\Omega \sigma]_{j k}$, which proves $(10.5 . \mathrm{b})$. Also, $8\{(\mathrm{D} \sigma) \cdot(\mathrm{D} \sigma)\}_{j k}$ equals

$$
2\left(C^{q}{ }_{p}^{r} \sigma_{r j}+C^{q}{ }_{j}^{r} \sigma_{p r}\right)\left(C^{p}{ }_{q}^{s} \sigma_{s k}+C_{k}^{p}{ }_{k}^{s} \sigma_{q s}\right),
$$

that is, $2 C^{q}{ }_{p}^{r} C^{p}{ }_{q}{ }^{s} \sigma_{r j} \sigma_{s k}-2 C_{r p}{ }^{q} C_{k s}{ }^{p} \sigma_{q}^{s} \sigma_{j}^{r}-2 C_{s q}{ }^{p} C_{j r}{ }^{q} \sigma_{p}^{r} \sigma_{k}^{s}+2 C_{j r}{ }^{q} \sigma_{p}^{r} C_{k s}{ }^{p} \sigma_{q}^{s}=$ $2 \sigma_{j}^{s} \sigma_{s k}-[\Omega \sigma]_{r k} \sigma_{j}^{r}-[\Omega \sigma]_{s j} \sigma_{k}^{s}+2\{\sigma \cdot \sigma\}_{j k}$, cf. (9.2), (9.6.a), (7.4), (7.8), (8.5.a) and (8.9.ii). This yields (10.5.c). Finally, (9.6.a) and (10.5.b) - (10.5.c) imply (10.5.d).

Next, for $\sigma, \tau \in \mathcal{T}$, applying (9.7.a) to $\mathrm{S}=\mathrm{D} \tau$ we obtain, from (10.3.b),

$$
4\langle\mathrm{D} \tau, \mathrm{D} \sigma\rangle=\langle\Omega \tau-2 \tau, \sigma\rangle .
$$

Remark 10.2. Another description of $\Phi$, obvious from (10.1), reads: $\Phi$ is diagonalizable, has the eigenvalues $1 / 2$ and -1 , with the respective eigenspaces consisting of those $\mathrm{S} \in \mathcal{S}$ for which $\left\langle u, \mathrm{~S}_{v} w\right\rangle$ is totally symmetric in $u, v, w \in \mathfrak{g}$ or, respectively, $\left\langle u, \mathrm{~S}_{v} w\right\rangle$ summed cyclically over $u, v, w \in \mathfrak{g}$ yields 0 .

Remark 10.3. We identify nondegenerate elements of $\mathcal{T}$ with left-invariant pseudo-Riemannian metrics $\gamma$ on G. According to Remark 5.2, the Levi-Civita connection of any such $\gamma$ is the unique $\nabla=\mathrm{D}+\mathrm{S}$, with $\mathrm{S} \in\left[\mathfrak{g}^{*}\right]^{\odot 2} \otimes \mathfrak{g}$, such that $\nabla \gamma=0$. Writing the last condition as $\mathrm{D} \gamma+\mathrm{S} \gamma=0$, and using (10.3), we get $\mathrm{D} \gamma=-\gamma \mathrm{S}$, that is, the classical Christoffel formula $\nabla=\mathrm{D}+\mathrm{S}$, for $\mathrm{S}=-\gamma^{-1}(\mathrm{D} \gamma)$. 


\section{Einstein and weakly-Einstein connections}

We adopt the assumptions and notations of Sections $7-9$, so that $\mathfrak{g}$ is our fixed semisimple Lie algebra over $\mathbb{F}=\mathbb{R}$ or $\mathbb{F}=\mathbb{C}$, associated with a real/complex Lie group $\mathrm{G}$, while $\left[\mathfrak{g}^{*}\right]^{\otimes 2} \otimes \mathfrak{g}$ is identified with the space of left-invariant connections on $\mathrm{G}$ (assumed holomorphic if $\mathbb{F}=\mathbb{C}$ ), and $\mathrm{D}+\mathcal{S}$ is its affine subspace formed by all $\nabla \in\left[\mathfrak{g}^{*}\right]^{\otimes 2} \otimes \mathfrak{g}$ which are torsion-free. Now, for $\mathcal{E}, \mathcal{U}, \mathcal{W}$ defined below,

$$
\mathcal{E} \subset \mathcal{U} \subset \mathcal{W} \subset \mathrm{D}+\mathcal{S} \subset\left[\mathfrak{g}^{*}\right]^{\otimes 2} \otimes \mathfrak{g} .
$$

Here $\mathcal{E}$ denotes the set of Einstein connections in $\mathfrak{g}$, that is, of the Levi-Civita connections of left-invariant Einstein metrics on G (again, assumed holomorphic when $\mathbb{F}=\mathbb{C}$ ). The subset $\mathcal{W}$ of $\mathrm{D}+\mathcal{S}$ consists, in turn, of weakly-Einstein connections in $\mathfrak{g}$, by which we mean all $\nabla \in \mathrm{D}+\mathcal{S}$ such that the left-invariant symmetric 2 -tensor field $\{\nabla \cdot \nabla\}$ on $\mathrm{G}$ is $\nabla$-parallel. Finally, $\mathcal{U} \subset \mathrm{D}+\mathcal{S}$ is formed by those $\nabla$ which are unimodular and have a $\nabla$-parallel Ricci tensor.

The inclusions $\mathcal{E} \subset \mathcal{U} \subset \mathcal{W}$ follow from Lemma 6.1(b) and (9.5). Next, we define a nonlinear mapping $\boldsymbol{H}: \mathcal{S} \rightarrow \mathcal{S}$ and a linear endomorphism $\Delta$ of $\mathcal{S}$ by

$$
\text { i) } \boldsymbol{H}(\mathrm{S})=4(\mathrm{D}+\mathrm{S})\{(\mathrm{D}+\mathrm{S}) \cdot(\mathrm{D}+\mathrm{S})\}, \quad \text { ii) } \Delta \mathrm{S}=8 \mathrm{D}\{\mathrm{D} \cdot \mathrm{S}\}+\mathrm{S} \beta \text {, }
$$

with $\mathcal{S}$ as in (7.1). According to (9.6.a), $4\{\mathrm{D} \cdot \mathrm{D}\}=\beta$, and hence

a) $4\{(\mathrm{D}+\mathrm{S}) \cdot(\mathrm{D}+\mathrm{S})\}=\beta+\sigma$, where b) $\sigma=8\{\mathrm{D} \cdot \mathrm{S}\}+4\{\mathrm{~S} \cdot \mathrm{S}\}$.

The operations used in (11.2.i) and (11.3.a) are the $\nabla$-gradient, cf. (8.3), and the first pairing in (9.1); thus, setting $\nabla=\mathrm{D}+\mathrm{S}$, we have

$$
\boldsymbol{H}(\mathrm{S})=4 \nabla\{\nabla \cdot \nabla\},
$$

while for the Ricci tensor $\rho^{\nabla}$ of $\nabla$, one obtains, from (7.5), (9.5) and (11.3),

$\boldsymbol{H}(\mathrm{S})=-4 \nabla \rho^{\nabla}$ and $\rho^{\nabla}=-(\beta+\sigma) / 4$ if $\mathbf{c S}=0$ and $\nabla=\mathrm{D}+\mathrm{S}$.

By (11.2) - (11.3), $\boldsymbol{H}(\mathrm{S})=\mathrm{D} \sigma+\mathrm{S} \sigma+\mathrm{S} \beta$, so that, as $\mathrm{D} \beta=0$ in (9.6.c),

$$
\boldsymbol{H}(\mathrm{S})=\Delta \mathrm{S}+4 \mathrm{D}\{\mathrm{S} \cdot \mathrm{S}\}+\mathrm{S} \sigma, \quad \text { with } \sigma=8\{\mathrm{D} \cdot \mathrm{S}\}+4\{\mathrm{~S} \cdot \mathrm{S}\} .
$$

Consequently, $\Delta$ equals the differential of $\boldsymbol{H}$ at 0 , that is

$$
\mathrm{d} \boldsymbol{H}_{0}=\Delta, \quad \text { while } \quad \boldsymbol{H}(0)=0,
$$

since (11.6) expresses $\boldsymbol{H}(\mathrm{S})$ as $\Delta \mathrm{S}$ plus some terms quadratic and cubic in $\mathrm{S}$.

The sets $\mathcal{U}$ and $\mathcal{W}$ appearing in (11.1) can now be described as follows:

i) $\mathcal{U}=\mathrm{D}+(\mathcal{Z} \cap \operatorname{Ker} \mathbf{c})=\mathcal{W} \cap \operatorname{Ker} \mathbf{c}, \quad$ and

ii) $\mathcal{W}=\mathrm{D}+\mathcal{Z}, \quad$ where iii) $\mathcal{Z}=\boldsymbol{H}^{-1}(0)$,

as (11.4) yields (11.8.ii), while (11.8.ii), (7.5), Remark 6.3 and (9.5) give (11.8.i).

Lemma 11.1. With the assumptions and notations as in Sections $7-9$, for any $\mathrm{S} \in \mathcal{S}$ and $\sigma \in \mathcal{T}$, the following two conditions are equivalent:

(a) $\Delta \mathrm{S}+4 \mathrm{D}\{\mathrm{S} \cdot \mathrm{S}\}+\mathrm{S} \sigma=0$, while $\mathbf{c S}=0$ and $\sigma=8\{\mathrm{D} \cdot \mathrm{S}\}+4\{\mathrm{~S} \cdot \mathrm{S}\}$, 
(b) $\nabla=\mathrm{D}+\mathrm{S}$ is a unimodular torsion-free connection in $\mathfrak{g}$ and its Ricci tensor, given by $\rho=-(\beta+\sigma) / 4$, is $\nabla$-parallel; consequently, $\nabla \in \mathcal{U}$.

Proof. First, (a) implies (b), since (7.5) with $\mathbf{c S}=0$ gives unimodularity of $\nabla$, and (11.5) - (11.6) show that $\rho=-(\beta+\sigma) / 4$ is $\nabla$-parallel. Conversely, assuming (b), we obtain (a) from (7.5), (9.5), (11.3) and (11.5) - (11.6).

Lemma 11.2. For the inner-product spaces (8.2), and $\Phi, \Delta$ as in (10.1), (11.2.ii),

(i) $\Phi, \Delta$ as well as $\mathrm{S} \mapsto \mathrm{S} \beta$ and $\mathrm{S} \mapsto \mathrm{D}\{\mathrm{D} \cdot \mathrm{S}\}$ are self-adjoint endomorphisms of $\mathcal{S}$,

(ii) $\Omega: \mathcal{T} \rightarrow \mathcal{T}$ defined by (8.8) is self-adjoint.

Proof. First, $\Phi$ is self-adjoint due to the first line of (3.6) and Remark 10.2; so is the endomorphism $\mathrm{S} \mapsto \mathrm{S} \beta$ equal, by (10.2.i), to $-2 \Phi-\mathrm{Id}$. The same follows for the operator $\mathrm{S} \mapsto \mathrm{D}\{\mathrm{D} \cdot \mathrm{S}\}$ obtained, up to a factor, as the composite of $\mathrm{S} \mapsto 2\{\mathrm{D} \cdot \mathrm{S}\}$ with its adjoint $\sigma \mapsto \mathrm{D} \sigma$ (see (9.7.a)). By (11.2.ii), the last two conclusions give (i) for $\Delta$. Finally, (8.1.i), (8.9.ii) and (7.8) imply symmetry of the expression $\langle\Omega \sigma, \tau\rangle=2 \tau^{j k} C_{j q p} C_{k r}{ }^{q} \sigma^{p r}$ in $\sigma, \tau \in \mathcal{T}$, proving (ii).

Remark 11.3. The quadratic mapping $\mathbf{K}: \mathcal{S} \times \mathcal{T} \rightarrow \mathcal{S} \times \mathcal{T}$ in Section 2 is given by $\boldsymbol{K}(\mathrm{S}, \sigma)=(\Delta \mathrm{S}+4 \mathrm{D}\{\mathrm{S} \cdot \mathrm{S}\}+\mathrm{S} \sigma, \sigma-8\{\mathrm{D} \cdot \mathrm{S}\}-4\{\mathrm{~S} \cdot \mathrm{S}\})$, and $\boldsymbol{L}(\mathrm{S}, \sigma)=(\Delta \mathrm{S}, \sigma)$. The correspondence (2.3) sends $\mathrm{D}+\mathrm{S}$ with $\boldsymbol{H}(\mathrm{S})=0$ to $(\mathrm{S}, \sigma)$, for $\sigma$ as in (11.6).

\section{The nondegeneracy condition}

We continue the discussion of Sections 7 - 11. By (11.2.ii), (10.5.a) and (10.5.b),

$$
\Delta(\mathrm{D} \sigma)=\mathrm{D}[(\Omega-\mathrm{Id}) \sigma] \quad \text { whenever } \sigma \in \mathcal{T} .
$$

Using the Christoffel isomorphism $\Phi$, given by (10.1), we obtain

$$
\operatorname{Ker} \Delta=\{\mathrm{D} \tau: \tau \in \operatorname{Ker}(\Omega-\mathrm{Id})\} .
$$

In fact, applying $\Phi$, we see that, by (11.2.ii), (10.3.a) with $\sigma=\{\mathrm{D} \cdot \mathrm{S}\}$, and (10.4.a), $\Delta \mathrm{S}=0$ if and only if $\mathrm{S}=\mathrm{D} \tau$ for $\tau=-8\{\mathrm{D} \cdot \mathrm{S}\}$, which is in turn equivalent to $\mathrm{S}=\mathrm{D} \tau$ for $\tau=-8\{\mathrm{D} \cdot(\mathrm{D} \tau)\}$, and hence, in view of (10.5.b), amounts to $\mathrm{S}=\mathrm{D} \tau$ for some $\tau \in \operatorname{Ker}(\Omega-\mathrm{Id})$. From (12.2) we further conclude that

$\tau \mapsto \mathrm{D} \tau$ is a linear isomorphism $\operatorname{Ker}(\Omega-\mathrm{Id}) \rightarrow \operatorname{Ker} \Delta$, with the inverse isomorphism $\operatorname{Ker} \Delta \ni \mathrm{S} \mapsto-8\{\mathrm{D} \cdot \mathrm{S}\} \in \operatorname{Ker}(\Omega-\mathrm{Id})$,

since, by (12.2), $\tau \mapsto \mathrm{D} \tau$ maps $\operatorname{Ker}(\Omega-\mathrm{Id})$ onto $\operatorname{Ker} \Delta$, while (10.5.b) implies its injectivity and describes its inverse.

From now on we will assume the nondegeneracy condition: for $\Omega$ with (8.8),

$$
\operatorname{Ker}(\Omega-\mathrm{Id}) \text { is a nondegenerate subspace of } \mathcal{T}
$$

in the sense of Section 3. This is no restriction of generality: in Lemma 17.1(f) we prove (12.4) for the Lie algebras of the groups (1.1), while the remaining simple Lie algebras have $\operatorname{Ker}(\Omega-\mathrm{Id})=\{0\}$ (see Remark 12.3), which again yields (12.4). 
Lemma 12.1. Condition (12.4) implies that
a) $\mathcal{T}=[(\Omega-\mathrm{Id})(\mathcal{T})] \oplus \operatorname{Ker}(\Omega-\mathrm{Id})$,
b) $\mathcal{S}=[\Delta(\mathcal{S})] \oplus \operatorname{Ker} \Delta$,

c) $\Delta: \Delta(\mathcal{S}) \rightarrow \Delta(\mathcal{S})$ is an isomorphism.

Proof. Formula (10.6) for $\sigma, \tau \in \operatorname{Ker}(\Omega-\mathrm{Id})$ becomes $4\langle\mathrm{D} \tau, \mathrm{D} \sigma\rangle=-\langle\tau, \sigma\rangle$, so that, by (12.3) and (12.4), Ker $\Delta$ is a nondegenerate subspace of $\mathcal{S}$. In view of Lemma 11.2, $\Delta: \mathcal{S} \rightarrow \mathcal{S}$ and $\Omega-\operatorname{Id}: \mathcal{T} \rightarrow \mathcal{T}$ are self-adjoint. Nondegeneracy of their kernels and (3.4.b) thus yield (12.5.a-b). Now (3.7) gives (12.5.c).

We will use the symbol $\Pi$ for both projection operators
a) $\Pi: \mathcal{T} \rightarrow \operatorname{Ker}(\Omega-\mathrm{Id})$,
b) $\Pi: \mathcal{S} \rightarrow \operatorname{Ker} \Delta$

arising, under the assumption (12.4), from the decompositions in (12.5.a-b).

Remark 12.2. For $\lambda \in \mathcal{T}$ we have $\mathrm{D}(\Pi \lambda)=\Pi(\mathrm{D} \lambda)$, the first (or, second) $\Pi$ being as in (12.6.a) (or, (12.6.b)). In fact, using (12.5.a) to write $\lambda=\tau+(\Omega-\mathrm{Id}) \sigma$, with $\tau \in \operatorname{Ker}(\Omega-\mathrm{Id})$ and $\sigma \in \mathcal{T}$, we obtain, from (12.1), $\mathrm{D} \lambda=\mathrm{D} \tau+\Delta(\mathrm{D} \sigma)$, where $\mathrm{D} \tau=\Pi(\mathrm{D} \lambda) \in \operatorname{Ker} \Delta$ by (12.2). Thus, $\Pi(\mathrm{D} \lambda)=\mathrm{D} \tau=\mathrm{D}(\Pi \lambda)$.

Remark 12.3. For simple Lie algebras other than the ones corresponding to (1.1) with $n=l+j \geq 3$ we have $\operatorname{Ker}(\Omega-\mathrm{Id})=0$. (See [7, Remark 5.4].) Then, by (12.2) and (11.7), 0 is isolated in $\boldsymbol{H}^{-1}(0)$. In other words, according to (11.8.ii) and (11.1), for those Lie algebras, D is an isolated point in both $\mathcal{W}$ and $\mathcal{E}$.

\section{The underlying real Lie algebras}

Denoting by $\mathfrak{g}_{\mathbb{R}}$ the underlying real Lie algebra of a given semisimple complex Lie algebra $\mathfrak{g}$ with the Killing form $\beta$, we see that, by (7.2) and (3.3),

$$
\text { the Killing form of } \mathfrak{g}_{\mathbb{R}} \text { equals } 2 \operatorname{Re} \beta \text {. }
$$

Thus, $\mathfrak{g}_{\mathbb{R}}$ is semisimple as well. The following lemma and remarks use

the spaces $\mathcal{T}_{\mathbb{R}}, \mathcal{S}_{\mathbb{R}}, \mathcal{Y}_{\mathbb{R}}$, the linear endomorphism $\Delta_{\mathbb{R}}: \mathcal{S}_{\mathbb{R}} \rightarrow \mathcal{S}_{\mathbb{R}}$, $\{\cdot\}_{\mathbb{R}}: \mathcal{Y}_{\mathbb{R}} \times \mathcal{Y}_{\mathbb{R}} \rightarrow \mathcal{T}_{\mathbb{R}}$, and the projection $\Pi_{\mathbb{R}}: \mathcal{S}_{\mathbb{R}} \rightarrow \operatorname{Ker} \Delta_{\mathbb{R}}$,

the $\mathfrak{g}_{\mathbb{R}}$-counterparts of $\mathcal{T}, \mathcal{S}, \mathcal{Y}, \Delta,\{\cdot\}, \Pi$ in (7.1), (11.2.ii), (9.1), (12.6.b) for $\mathfrak{g}$.

Lemma 13.1. For the real space $\mathcal{T}^{\prime}$ of all Hermitian sesquilinear forms $\mathfrak{g} \times \mathfrak{g} \rightarrow \mathbb{C}$,

(a) $\mathcal{T}_{\mathbb{R}}=[\operatorname{Re} \mathcal{T}] \oplus\left[\operatorname{Re} \mathcal{T}^{\prime}\right]$, where the summands are $\mathbb{R}$-isomorphic images of $\mathcal{T}$ and $\mathcal{T}^{\prime}$ under the operator $\sigma \mapsto \operatorname{Re} \sigma$,

(b) $\operatorname{Re} \mathcal{T}^{\prime} \subset \operatorname{Ker} \Omega_{\mathbb{R}}$,

(c) $\Omega_{\mathbb{R}}(\operatorname{Re} \sigma)=\operatorname{Re}(\Omega \sigma)$ for all $\sigma \in \mathcal{T}$, so that $\Omega_{\mathbb{R}}$ leaves the summand $\operatorname{Re} \mathcal{T}$ invariant and $\Omega_{\mathbb{R}}: \operatorname{Re} \mathcal{T} \rightarrow \operatorname{Re} \mathcal{T}$ corresponds, under the isomorphic identification $\mathcal{T} \rightarrow \operatorname{Re} \mathcal{T}$ in (a), to $\Omega: \mathcal{T} \rightarrow \mathcal{T}$, 
(d) $\operatorname{Ker}\left(\Omega_{\mathbb{R}}-\mathrm{Id}\right)$ is contained in the summand $\operatorname{Re} \mathcal{T}$, cf. (a), and arises as the image of $\operatorname{Ker}(\Omega-\mathrm{Id}) \subset \mathcal{T}$ under the isomorphism $\mathcal{T} \ni \sigma \mapsto \operatorname{Re} \sigma \in \operatorname{Re} \mathcal{T}$.

Proof. If a $\mathbb{C}$-linear endomorphism $\Sigma$ of $\mathfrak{g}$ is related to $\sigma \in \mathcal{T}$ via (7.3), taking the real parts of both sides of (7.3), with $\langle\rangle=,\beta$, we see that, by (13.1), the same relation holds, in $\mathfrak{g}_{\mathbb{R}}$, between $\Sigma$ and $2 \operatorname{Re} \sigma \in \operatorname{Re} \mathcal{T}$. Now (8.8), its real-part version, and (3.3) yield (c). Next, given $\sigma \in \mathcal{T}^{\prime}$, let $\Sigma: \mathfrak{g}_{\mathbb{R}} \rightarrow \mathfrak{g}_{\mathbb{R}}$ be associated with $\operatorname{Re} \sigma$ as in (7.3) for $\mathfrak{g}_{\mathbb{R}}$ and $2 \operatorname{Re} \beta$ instead of $\mathfrak{g}$ and $\beta$. The $\mathbb{R}$-linear endomorphism $\mathfrak{g}_{\mathbb{R}} \rightarrow \mathfrak{g}_{\mathbb{R}}$ of multiplication by $\mathrm{i}$ is $(\operatorname{Re} \beta)$-self-adjoint, so that $0=$ $\operatorname{Re}[\sigma(\mathrm{i} v, w)+\sigma(v, \mathrm{i} w)]=2 \operatorname{Re} \beta(\Sigma \mathrm{i} v+\mathrm{i} \Sigma v, w)$ for all $v, w \in \mathfrak{g}$, and $\Sigma$ is $\mathbb{C}$-antilinear. As (8.8) and (3.3) imply that $\left[\Omega_{\mathbb{R}}(\operatorname{Re} \sigma)\right](v, w)$ equals $4 \operatorname{Re} \operatorname{tr}^{\mathbb{C}}[(\operatorname{Ad} v)(\operatorname{Ad} w) \Sigma]$, the final clause of Remark 3.1 gives $\Omega_{\mathbb{R}}(\operatorname{Re} \sigma)=0$, proving (b). Finally, since any $\sigma \in \mathcal{T}$ or $\sigma \in \mathcal{T}^{\prime}$ is uniquely determined by its real part, (a) follows, while (d) is a trivial consequence of (a), (b) and (c).

Remark 13.2. One has $2 \nabla(\operatorname{Re} \sigma)=\nabla \sigma$ if $\nabla: \mathfrak{g} \times \mathfrak{g} \rightarrow \mathfrak{g}$ is a (complex-bilinear) connection in a semisimple complex Lie algebra $\mathfrak{g}$ and $\sigma \in \mathcal{T}$, with $\nabla \sigma$ as in (8.3), where $\nabla(\operatorname{Re} \sigma)$ is analogously defined for $\mathfrak{g}_{\mathbb{R}}$, so that $\operatorname{Re} \sigma \in \mathcal{T}_{\mathbb{R}}$ (notation of Lemma 13.1) and $\nabla$ is treated as a (real-bilinear) connection in $\mathfrak{g}_{\mathbb{R}}$.

To see this, apply 2 Re to both sides in (8.3) and use (13.1) with $\beta=\langle$,$\rangle .$

Remark 13.3. Given $\mathfrak{g}$ and $\mathfrak{g}_{\mathbb{R}}$ as above, let us use the notation of (13.2).

(i) The real part of a (holomorphic) metric $\gamma$ in $\mathfrak{g}$ is a (pseudo-Riemannian) metric in $\mathfrak{g}_{\mathbb{R}}$, and both metrics have the same Levi-Civita connection.

(ii) If $\rho$ is the Ricci tensor of the Levi-Civita connection of a metric in $\mathfrak{g}$, then $\nabla$ viewed as a connection in $\mathfrak{g}_{\mathbb{R}}$ has Ricci tensor $2 \operatorname{Re} \rho$.

(iii) The real part of every Einstein metric in $\mathfrak{g}$ is an Einstein metric in $\mathfrak{g}_{\mathbb{R}}$.

(iv) Every Einstein connection in $\mathfrak{g}$ is also an Einstein connection in $\mathfrak{g}_{\mathbb{R}}$.

(v) $\{\nabla \cdot \tilde{\nabla}\}_{\mathbb{R}}=2 \operatorname{Re}\{\nabla \cdot \tilde{\nabla}\}$ whenever $\nabla, \tilde{\nabla} \in \mathcal{Y} \subset \mathcal{Y}_{\mathbb{R}}$.

(vi) $\operatorname{Ker} \Delta_{\mathbb{R}}=\operatorname{Ker} \Delta$, so that any element $\mathrm{S}: \mathfrak{g}_{\mathbb{R}} \times \mathfrak{g}_{\mathbb{R}} \rightarrow \mathfrak{g}_{\mathbb{R}}$ of $\operatorname{Ker} \Delta_{\mathbb{R}}$, originally assumed real-bilinear, must actually be complex-bilinear.

(vii) $\Delta: \mathcal{S} \rightarrow \mathcal{S}$ is the restriction of $\Delta_{\mathbb{R}}: \mathcal{S}_{\mathbb{R}} \rightarrow \mathcal{S}_{\mathbb{R}}$ to $\mathcal{S} \subset \mathcal{S}_{\mathbb{R}}$.

(viii) If $\mathrm{S} \in \mathcal{S}_{\mathbb{R}}$ and $\Delta_{\mathbb{R}} \mathrm{S} \in \mathcal{S}$, then $\mathrm{S} \in \mathcal{S}$.

In fact, (i) is obvious from Remarks 5.2 and 13.2 (the latter for $\sigma=\gamma$ ); (ii) from Lemma 6.1(d) combined with (3.3) (where the former can be applied in view of Remark 6.3 and Lemma 6.1(b)); (iii) from (i) - (ii); (iv) from (i) and (iii); (v) from (9.1) and (3.3). Next, elements of $\operatorname{Ker} \Delta_{\mathbb{R}}$ coincide, by (12.2) for $\mathfrak{g}_{\mathbb{R}}$, with the D-gradients $\mathrm{D} \tau$, in $\mathfrak{g}_{\mathbb{R}}$, of all $\tau \in \operatorname{Ker}\left(\Omega_{\mathbb{R}}-\mathrm{Id}\right)$. According to Lemma 13.1(d), such $\tau$ are precisely the same as all $\operatorname{Re} \sigma$ for $\sigma \in \operatorname{Ker}(\Omega-\mathrm{Id})$. As Remark 13.2 with $\nabla=\mathrm{D}$ gives $2 \mathrm{D} \tau=2 \mathrm{D}(\operatorname{Re} \sigma)=\mathrm{D} \sigma$, (vi) follows from (12.2). Furthermore, (vii) is an immediate consequence of (11.2.ii), (v), (13.1) and Remark 13.2. 
Finally, let $\mathrm{S} \in \mathcal{S}_{\mathbb{R}}$ and $\Delta_{\mathbb{R}} \mathrm{S} \in \mathcal{S}$. By (12.5.b), $\Delta_{\mathbb{R}} \mathrm{S}-\Delta \tilde{\mathrm{S}} \in \operatorname{Ker} \Delta$ for some $\tilde{\mathrm{S}} \in \mathcal{S}$. From (vi) and (vii) one thus has $\Delta_{\mathbb{R}}(\mathrm{S}-\tilde{\mathrm{S}}) \in \operatorname{Ker} \Delta_{\mathbb{R}}$. Applying (12.5.b-c) to $\mathfrak{g}_{\mathbb{R}}$ rather than $\mathfrak{g}$, we now conclude that $\Delta_{\mathbb{R}}(\mathrm{S}-\tilde{\mathrm{S}})=0$, and so $\mathrm{S}-\tilde{\mathrm{S}} \in \operatorname{Ker} \Delta_{\mathbb{R}}=\operatorname{Ker} \Delta \subset \mathcal{S}$ (cf. (vi)). Hence $\mathrm{S} \in \mathcal{S}$.

\section{Complexifications}

All vector spaces (and Lie algebras) are assumed here to be finite-dimensional.

By a real form of a complex vector space (or, a complex Lie algebra) $\mathfrak{g}^{\mathbb{C}}$ we mean any real subspace (or, real Lie subalgebra) $\mathfrak{g} \subset \mathfrak{g}^{\mathbb{E}}$ such that, as a real vector space, $\mathfrak{g}^{\mathbb{C}}=\mathfrak{g} \oplus \mathfrak{i g}$. This is, in an obvious sense, equivalent to requiring that $\mathfrak{g}^{\mathbb{C}}$ be the vector-space (or, Lie-algebra) complexification of $\mathfrak{g}$. Whenever $l+j=n$,

$$
\mathfrak{s l}(n, \mathbb{R}), \quad \mathfrak{s u}(l, j) \quad \text { and } \mathfrak{s l}(n / 2, \mathbb{H}) \text { are real forms of } \mathfrak{s l}(n, \mathbb{C}),
$$

the last one for even $n$ only. Here, by definition, $\mathfrak{s l}(k, \mathbb{H})$ consists of those $\mathbb{H}$-linear endomorphisms of $\mathbb{H}^{k}$ which are also $\mathbb{C}$-traceless in the sense of Remark 3.2. Thus, with $\mathfrak{g}=\mathfrak{s l}(k, \mathbb{H})$, and with $\mathfrak{g}^{\mathbb{C}} \approx \mathfrak{s l}(2 k, \mathbb{C})$ denoting the Lie algebra of all traceless $\mathbb{C}$-linear endomorphisms of $\mathbb{H}^{k}$, the $( \pm 1)$-eigenspace decomposition of $\mathfrak{g}^{\mathbb{C}}$ under the involution $a \mapsto-\mathrm{J} a \mathrm{~J}$, for $\mathrm{J}$ as in Remark 3.2, reads $\mathfrak{g}^{\mathbb{C}}=\mathfrak{g} \oplus \mathrm{ig}$. (The components $a, b \in \mathfrak{g}$ of any $a+\mathrm{i} b \in \mathfrak{g}^{\mathbb{C}}$ are indeed $\mathbb{C}$-traceless, since so is $a+\mathrm{i} b$, while the $\mathbb{C}$-traces of $a$ and $b$ are real by Remark 3.2.)

The four Lie algebras appearing in (14.1) are subsets of $\mathfrak{g l}(n, \mathbb{C})$. Any finite product $w$ of their elements is therefore a complex $n \times n$ matrix, and we denote by $\operatorname{tr} w$ its matrix trace. Hence, for $a, b, w \in \mathfrak{g l}(n, \mathbb{C})$, one has the inner product $(a, b)$ as in (3.1), and the traceless part $(w)_{0}$, with

$$
\text { i) }(a, b)=\operatorname{tr} a b, \quad \text { ii) }(w)_{0}=w-(\operatorname{tr} w) \operatorname{Id} / n .
$$

Given a real form $\mathfrak{g}$ of an $m$-dimensional complex vector space $\mathfrak{g}^{\mathbb{C}}$,

any $\mathbb{R}$-basis $e_{1}, \ldots, e_{m}$ of $\mathfrak{g}$ is at the same time a $\mathbb{C}$-basis of $\mathfrak{g}^{\mathbb{L}}$,

leading, as in the lines following (7.5), to the corresponding components of

$$
\text { any } \mathbb{C} \text {-(bi)linear mapping from } \mathfrak{g}^{\mathbb{C}} \text { valued in } \mathbb{C} \text { or } \mathfrak{g}^{\mathbb{C}} \text {. }
$$

Remark 14.1. A mapping (14.4) is the unique $\mathbb{C}$-(bi)linear extension of an $\mathbb{R}$ valued or a $\mathfrak{g}$-valued mapping from $\mathfrak{g}$ if and only if the former has the same components as the latter relative to some/any pair of bases as in (14.3). We will denote both mappings by the same symbol.

Example 14.2. For an $\mathbb{R}$-linear endomorphism $\Sigma$ of $\mathfrak{g}$, its $\mathbb{C}$-linear extension $\Sigma$ to $\mathfrak{g}^{\mathbb{C}}$, and a pair of bases of type (14.3),

$$
\text { the } \mathbb{C} \text {-trace of } \Sigma: \mathfrak{g}^{\mathbb{C}} \rightarrow \mathfrak{g}^{\mathbb{C}} \text { equals the } \mathbb{R} \text {-trace of } \Sigma: \mathfrak{g} \rightarrow \mathfrak{g},
$$


as both traces coincide with $\Sigma_{k}^{k}$, where $\Sigma e_{j}=\Sigma_{j}^{k} e_{k}$. In the case of Lie algebras,

the Killing form of $\mathfrak{g}^{\mathbb{C}}$ is $\beta: \mathfrak{g}^{\mathbb{C}} \times \mathfrak{g}^{\mathbb{C}} \rightarrow \mathbb{C}$, the $\mathbb{C}$-bilinear extension to $\mathfrak{g}^{\mathbb{C}}$ of the Killing form $\beta: \mathfrak{g} \times \mathfrak{g} \rightarrow \mathbb{R}$ of $\mathfrak{g}$,

since, due to (7.2), (3.1), and (14.5) applied to $\Sigma=(\operatorname{Ad} v) \operatorname{Ad} w$, the latter Killing form arises as the restriction of the former from $\mathfrak{g}^{\mathbb{C}}$ to $\mathfrak{g}$. In addition, obviously,

the Lie bracket $[]:, \mathfrak{g}^{\mathbb{C}} \times \mathfrak{g}^{\mathbb{C}} \rightarrow \mathfrak{g}^{\mathbb{C}}$ of $\mathfrak{g}^{\mathbb{C}}$ is the unique

$\mathbb{C}$-bilinear extension of the Lie bracket $[]:, \mathfrak{g} \times \mathfrak{g} \rightarrow \mathfrak{g}$.

We could also have derived (14.6) from (14.7) via Remark 14.1, using the component formula $\beta_{j k}=C_{p j}{ }^{q} C_{q k}{ }^{p}$ in (9.6.a) valid for both $\mathfrak{g}$ and $\mathfrak{g}^{\mathbb{C}}$.

Let $\mathfrak{g}$ be a real form of a complex Lie algebra $\mathfrak{g}^{\mathbb{C}}$. We denote by $\mathcal{T}^{\mathbb{C}}$ the second complex symmetric power of the complex dual space of $\mathfrak{g}^{\mathbb{C}}$, that is, the analog for $\mathfrak{g}^{\mathbb{C}}$ of the space $\mathcal{T}$ associated with $\mathfrak{g}$ as in (7.1). Our notation is consistent with the fact that $\mathcal{T}^{\mathbb{C}}$ may be treated as the complexification of $\mathcal{T}$. In other words, $\mathcal{T}$ can be naturally identified with a real form of $\mathcal{T}^{\mathbb{C}}$, so that

$$
\mathcal{T} \subset \mathcal{T}^{\mathbb{C}} \text { and } \mathcal{T}^{\mathbb{C}}=\mathcal{T} \oplus i \mathcal{T}
$$

Specifically, any $\tau \in \mathcal{T}$, which is a symmetric bilinear form $\tau: \mathfrak{g} \times \mathfrak{g} \rightarrow \mathbb{R}$, is identified with its $\mathbb{C}$-bilinear extension $\tau: \mathfrak{g}^{\mathbb{C}} \times \mathfrak{g}^{\mathbb{C}} \rightarrow \mathbb{C}$. Thus, $\mathcal{T} \subset \mathcal{T}^{\mathbb{C}}$ consists of all symmetric $\mathbb{C}$-bilinear forms $\tau: \mathfrak{g}^{\mathbb{C}} \times \mathfrak{g}^{\mathbb{C}} \rightarrow \mathbb{C}$ for which $\tau(v, w)$ is real whenever $v, w \in \mathfrak{g}$ (or, equivalently, $\tau_{j k} \in \mathbb{R}$ in a basis of type (14.3)).

Lemma 14.3. For a real Lie algebra $\mathfrak{g}$ and its complexification $\mathfrak{g}^{\mathbb{C}}=\mathfrak{g} \oplus \mathrm{i} \mathfrak{g}$,

(i) $\mathfrak{g}$ is semisimple if and only if so is $\mathfrak{g}^{\mathbb{C}}$.

Let $\mathfrak{g}$ now be semisimple. Then

(ii) the analog for $\mathfrak{g}^{\mathbb{C}}$ of $\Omega: \mathcal{T} \rightarrow \mathcal{T}$, given in $\mathfrak{g}$ by (8.8), is the unique $\mathbb{C}$-linear extension $\Omega: \mathcal{T}^{\mathbb{C}} \rightarrow \mathcal{T}^{\mathbb{C}}$ of $\Omega$ to $\mathcal{T}^{\mathbb{C}}$, cf. (14.8),

(iii) the operations $\{\cdot\}, \circledast: \mathcal{T}^{\mathbb{C}} \times \mathcal{T}^{\mathbb{C}} \rightarrow \mathcal{T}^{\mathbb{C}}$ defined for $\mathfrak{g}^{\mathbb{C}}$ as in (9.1) and the lines following Lemma 10.1 are the $\mathbb{C}$-linear extensions of their counterparts in $\mathfrak{g}$.

Proof. By (14.6) and Remark 14.1, both Killing forms have the same components $\beta_{j k}$ in bases of type (14.3), which yields (i). Assertions (ii) - (iii) are in turn immediate from Remark 14.1, applied, instead of a basis $e_{1}, \ldots, e_{m}$ of $\mathfrak{g}$, to the basis of $\mathcal{T}=\left[\mathfrak{g}^{*}\right]^{\odot 2}$ formed by suitable symmetric products of the basis of $\mathfrak{g}^{*}$ dual to $e_{1}, \ldots, e_{m}$. The components in question are given by the equality $[\Omega \sigma]_{j k}=$ $Z_{j k}^{r s} \sigma_{r s}$, two lines after (8.9), along with (9.2) and $2(\sigma \circledast \tau)_{j k}=\sigma_{j s} \tau_{k}^{s}+\tau_{j s} \sigma_{k}^{s}$. 


\section{Traces and Lie subalgebras}

Let $\mathbb{F}=\mathbb{R}$ or $\mathbb{F}=\mathbb{C}$. For the linear endomorphism $v \mapsto u v w$ of $\mathfrak{g l}(n, \mathbb{F})$,

the $\mathfrak{g l}(n, \mathbb{F})$-trace of $v \mapsto u v w$ equals $(\operatorname{tr} u) \operatorname{tr} w$,

with any fixed $u, w \in \mathfrak{g l}(n, \mathbb{F})$. In fact, $(\operatorname{tr} u) \operatorname{tr} w$ is the sum of the diagonal terms of the coefficient matrix $u_{p}^{s} w_{q}^{r}$ in the component expression $(u v w)_{q}^{s}=u_{p}^{s} v_{r}^{p} w_{q}^{r}$.

Remark 15.1. Suppose that $\mathcal{V}$ is a vector space with $\operatorname{dim}_{\mathbb{F}} \mathcal{V}<\infty$.

(i) If $\mathrm{A}(\mathcal{V}) \subset \tilde{\mathcal{V}}$ for a linear endomorphism $\mathrm{A}: \mathcal{V} \rightarrow \mathcal{V}$ and a subspace $\tilde{\mathcal{V}} \subset \mathcal{V}$, then the $\mathcal{V}$-trace of A equals the $\tilde{\mathcal{V}}$-trace of its restriction to $\tilde{\mathcal{V}}$.

(ii) Given a linear functional $\alpha \in \mathcal{V}^{*}$, and a vector $w \in \mathcal{V}$, one clearly has $\operatorname{tr}(\alpha \otimes w)=\alpha(w)$, where $\alpha \otimes w$ acts on $v \in \mathcal{V}$ by $v \mapsto \alpha(v) w$.

Remark 15.2. In the next two sections traces of linear endomorphisms A of $\mathfrak{s l}(n, \mathbb{F})$, where $\mathbb{F}=\mathbb{R}$ or $\mathbb{F}=\mathbb{C}$, will be evaluated as follows:

(a) write $\mathrm{A}$ as an endomorphism of $\mathfrak{g l}(n, \mathbb{F})$, valued in $\mathfrak{s l}(n, \mathbb{F})$,

(b) find the $\mathfrak{g l}(n, \mathbb{F})$-trace of the latter, using either (15.1), or Remark 15.1(ii),

(c) note that, by Remark 15.1(i), this is also the $\mathfrak{s l}(n, \mathbb{F})$-trace of $\mathrm{A}$.

Lemma 15.3. If $\mathfrak{g}$ is one of the four Lie algebras in (14.1), $a, b \in \mathfrak{g}$, while $\langle$,$\rangle and (, )$ are the Killing form and the inner product, with (7.2) and (14.2), then $\langle a, b\rangle=2 n(a, b)$.

Proof. Let $a, b \in \mathfrak{g l}(n, \mathbb{F})$, for $\mathbb{F}=\mathbb{R}$ or $\mathbb{F}=\mathbb{C}$. As $(\operatorname{Ad} a) \operatorname{Ad} b$ sends any $v \in \mathfrak{g l}(n, \mathbb{F})$ to $v \mapsto a b v-a v b-b v a+v b a$, its $\mathfrak{g l}(n, \mathbb{F})$-trace equals, from (15.1), $2 n(a, b)-2(\operatorname{tr} a) \operatorname{tr} b$. By Remark 15.1(i), if $a, b \in \mathfrak{s l}(n, \mathbb{F})$, this is also the $\mathfrak{s l}(n, \mathbb{F})$ trace of $(\operatorname{Ad} a) \operatorname{Ad} b$, which proves our claim in the case where $\mathfrak{g}=\mathfrak{s l}(n, \mathbb{F})$.

Our assertion for $\mathfrak{g}=\mathfrak{s u}(l, j)$ and $\mathfrak{g}=\mathfrak{s l}(n / 2, \mathbb{H})$ now follows from (14.6) applied to either choice of $\mathfrak{g}$ and to $\mathfrak{g}^{\mathbb{C}}=\mathfrak{s l}(n, \mathbb{C})$, cf. (14.1).

\section{The special linear and pseudo-unitary Lie algebras}

In this and the following sections, $(\mathfrak{g}, \mathbb{F}, \varepsilon)$ will always be one of the triples

$$
\begin{aligned}
& (\mathfrak{s l}(n, \mathbb{R}), \mathbb{R}, 1),(\mathfrak{s l}(n, \mathbb{C}), \mathbb{C}, 1),(\mathfrak{s l}(n, \mathbb{C}), \mathbb{C}, \mathrm{i}), \text { with } n \geq 2, \\
& (\mathfrak{s u}(l, j), \mathbb{R}, \mathrm{i}) \text { and, for even } n \geq 2 \text { only, }(\mathfrak{s l}(n / 2, \mathbb{H}), \mathbb{R}, 1),
\end{aligned}
$$

formed by a simple Lie algebra $\mathfrak{g}$ over $\mathbb{F}=\mathbb{R}$ or $\mathbb{F}=\mathbb{C}$ (see (14.1)), the scalar field $\mathbb{F}$ itself, and a fixed scalar $\varepsilon \in \mathbb{F}$, equal to 1 or i. Here $j, l$ are fixed integers with $l \geq j \geq 0$ and $l+j=n$. As before, $\beta=\langle$,$\rangle denotes the \mathbb{F}$-bilinear Killing form of $\mathfrak{g}$, defined by (7.2), so that $\beta \in \mathcal{T}$ for the space $\mathcal{T}$ in (7.1).

With any $a, b \in \mathfrak{g}$ we associate elements $\tau_{a}, \theta_{a, b}, \mu_{a, b}$ of $\mathcal{T}$, related as in (7.3) to the $\mathbb{F}$-linear endomorphisms of $\mathfrak{g}$ sending each $v \in \mathfrak{g}$, respectively, to

$$
\varepsilon(a v+v a)_{0}, \quad \varepsilon^{2}[(a, v) b+(b, v) a] /(2 n) \text { and } \varepsilon^{2}(a v b+b v a)_{0} / 2,
$$

where $($,$) and the traceless part ()_{0}$ are as in (14.2). 
Note that the values (16.2) all lie in $\mathfrak{g}$ due to our choice of $\varepsilon$. In addition, the operators assigning these values to $v$ are easily seen to be self-adjoint for the inner product (14.2.i). Their self-adjointness relative to the Killing form $\beta$ of $\mathfrak{g}$ is now immediate from Lemma 15.3. Next, whenever $a \in \mathfrak{g}$, we set

$$
c=\varepsilon\left(a^{2}\right)_{0}, \quad d=\varepsilon^{2}\left(a^{3}\right)_{0}, \quad \theta_{a}=\theta_{a, a}, \quad \mu_{a}=\mu_{a, a}, \quad \xi=\varepsilon^{2}(a, a) / n,
$$

with $(a, a)=\operatorname{tr} a^{2}$. Thus, in the sense of (16.2) and the lines preceding it,

$$
\begin{aligned}
& \tau_{a} \text { corresponds to } v \mapsto \varepsilon(a v+v a)_{0}, \quad \beta \text { to Id, } \\
& \theta_{a} \text { to } v \mapsto \varepsilon^{2}(a, v) a / n, \text { and } \mu_{a} \text { to } v \mapsto \varepsilon^{2}(a v a)_{0} .
\end{aligned}
$$

In proofs of some equalities involving $\theta_{a, b}$ and $\mu_{a, b}$ we may assume that $b=a$, as

$$
\text { the dependence of } \theta_{a, b} \text { and } \mu_{a, b} \text { on } a, b \text { is bilinear and symmetric. }
$$

Also, if $\xi$ (in (16.3)) and $\varepsilon(a, c) / n$ stand for the corresponding multiples of Id,

i) $\varepsilon a^{2}=c+\varepsilon^{-1} \xi, \quad$ ii) $\varepsilon^{2} a^{3}=d+\varepsilon(a, c) / n$, with $(a, c)=\operatorname{tr} a c$.

Using (8.8), where we may set $v=w,(8.9 . \mathrm{i})$ and (16.4), the three steps of Remark 15.2, formula (16.6.i), and then Lemma 15.3, we obtain, for $a \in \mathfrak{g}$,

$$
n^{2} \Omega \theta_{a}=\tau_{c}-2 \mu_{a}+2 \xi \beta, \quad \Omega \mu_{a}=-2 \theta_{a}, \quad \Omega \tau_{a}=\tau_{a}, \quad \Omega \beta=2 \beta .
$$

More precisely, we just proved (16.7) for the first three triples in (16.1). It follows, however, that (16.7) is satisfied by the other two triples as well. In fact, according to (14.1), relations (14.6), (14.8) and assertions (ii) - (iii) in Lemma 14.3 hold for $\mathfrak{g}=\mathfrak{s u}(l, j)$ or $\mathfrak{g}=\mathfrak{s l}(n / 2, \mathbb{H})$, and $\mathfrak{g}^{\mathbb{C}}=\mathfrak{s l}(n, \mathbb{C})$. Lemma 14.3(ii), combined with Remark 16.1 below, now shows that the $\Omega$-images of $\tau_{a}, \theta_{a, b}, \mu_{a, b}$ in $\mathfrak{s u}(l, j)$ or $\mathfrak{s l}(n / 2, \mathbb{H})$ coincide with their $\Omega$-images in $\mathfrak{s l}(n, \mathbb{C})$.

Note that, if $x, y, z \in \mathbb{F}$ and $\lambda=x \tau_{a}+n^{2} y \theta_{a}+z \mu_{a}$, (16.7) yields

$$
(\mathrm{Id}-\Omega) \lambda=\left(n^{2} y+2 z\right) \theta_{a}+(2 y+z) \mu_{a}-2 \xi y \beta-y \tau_{c} .
$$

Remark 16.1. By (14.6), the elements $\tau_{a}, \theta_{a, b}, \mu_{a, b}$ of $\mathcal{T}^{\mathbb{C}}$ associated with the triple $(\mathfrak{s l}(n, \mathbb{C}), \mathbb{C}, \mathrm{i})$, or $(\mathfrak{s l}(n, \mathbb{C}), \mathbb{C}, 1)$, are the unique $\mathbb{C}$-bilinear extensions of $\tau_{a}, \theta_{a, b}, \mu_{a, b}$ defined for $(\mathfrak{s u}(l, j), \mathbb{R}, \mathrm{i})$ or, respectively, for $(\mathfrak{s l}(n / 2, \mathbb{H}), \mathbb{R}, 1)$. Thus, $\tau_{a}, \theta_{a, b}, \mu_{a, b}$ lie in the real form $\mathcal{T} \subset \mathcal{T}^{\mathbb{C}}$ appearing in (14.8).

\section{The curvature spectra of the Lie algebras in (16.1)}

Whenever $(\mathfrak{g}, \mathbb{F}, \varepsilon)$ is one of the triples (16.1), $n=l+j \geq 2$, and $a, b \in \mathfrak{g}$,

(i) $n\left\langle\tau_{a}, \tau_{a}\right\rangle=2 \varepsilon^{2}\left(n^{2}-4\right)(a, a)$, with $\langle\rangle,, \tau_{a},($, ) as in (8.1.i), (16.4), (14.2.i),

(ii) for $n \geq 3$ the operator $\mathfrak{g} \ni a \mapsto \tau_{a} \in \mathcal{T}$ is injective,

(iii) for $n=2$ one has $\tau_{a}=0$ and $2 \theta_{a, b}=\mu_{a, b}+\varepsilon^{2}(a, b) \beta / 2$, cf. (16.2) and (7.2). 
In fact, if $\mathfrak{g}=\mathfrak{s l}(n, \mathbb{F})$, (i) follows from the three steps of Remark 15.2, as $\left\langle\tau_{a}, \tau_{a}\right\rangle$ is the trace of the endomorphism of $\mathfrak{g}$ sending $v$ to $\varepsilon^{2}\left(a(a v+v a)_{0}+(a v+v a)_{0} a\right)_{0}$, that is, to $\varepsilon^{2}$ times $a^{2} v+v a^{2}+2 a v a-4\left[(a, v) a+\left(a^{2}, v\right) \mathrm{Id}\right] / n$. For the other choices of $\mathfrak{g}$, (i) is obvious from (14.1) and Remark 16.1, combined with (14.5) since, again, $\left\langle\tau_{a}, \tau_{a}\right\rangle$ equals the trace of the square of the endomorphism corresponding to $\tau_{a}$ in (16.4). Next, the operator in (ii) is injective: by (i), it pulls some symmetric bilinear form in $\mathcal{T}$ back to the nondegenerate form $($,$) in \mathfrak{g}$, cf. Lemma 15.3. Finally, proving (iii) amounts, for reasons of symmetry as in (16.5), to showing that $a^{2}$ is a multiple of $\operatorname{Id}$ and $2 \theta_{a}=\mu_{a}+\varepsilon^{2}(a, a) \beta / 2$. This is easily verified if $a$ is the diagonal matrix $\operatorname{diag}(1,-1)$. The case of arbitrary $a$ is now immediate as matrices conjugate to multiples of $\operatorname{diag}(1,-1)$ form a dense subset of $\mathfrak{s l}(2, \mathbb{F})$, consisting of all traceless matrices with two distinct eigenvalues.

Lemma 17.1. If $(\mathfrak{g}, \mathbb{F}, \varepsilon)$ is one of the triples (16.1) and $n=l+j \geq 2$, then the endomorphism $\Omega$ of $\mathcal{T}=\left[\mathfrak{g}^{*}\right]^{\odot 2}$ defined by (8.8) is diagonalizable and has the following ordered system spec $[\Omega]$ of eigenvalues and mult $[\Omega]$ of the corresponding multiplicities.

(a) $\operatorname{spec}[\Omega]=(-1,2)$ and $\operatorname{mult}[\Omega]=(5,1)$, when $n=2$,

(b) $\operatorname{spec}[\Omega]=(-2 / 3,1,2)$ and mult $[\Omega]=(27,8,1)$ when $n=3$,

(c) $\operatorname{spec}[\Omega]=(-2 / n, 2 / n, 1,2)$ and mult $[\Omega]=\left(d^{-}, d^{+}, n^{2}-1,1\right)$ for $n \geq 4$, where $d^{ \pm}=n^{2}(n \mp 3)(n \pm 1) / 4$.

Furthermore, with $\tau_{a}$ and the Killing form $\beta$ as in (16.4),

(d) for $n \geq 3$, the assignment $a \mapsto \tau_{a}$ is a linear isomorphism $\mathfrak{g} \rightarrow \operatorname{Ker}(\Omega-\mathrm{Id})$,

(e) $\operatorname{Ker}(\Omega-2 \mathrm{Id})=\mathbb{F} \beta$,

(f) $\operatorname{Ker}(\Omega-\mathrm{Id})$ is a nondegenerate subspace of $\mathcal{T}$, that is, (12.4) holds.

Proof. We define $\iota_{a, b}^{ \pm} \in \mathcal{T}$ by $\iota_{a, b}^{ \pm}=n \theta_{a, b} \mp \mu_{a, b}-(n \mp 2)^{-1} \tau_{e}-[n(n \mp 1)]^{-1} \varepsilon^{2}(a, b) \beta$, where $e=\varepsilon(a b+b a)_{0} / 2$. (Notation of $(16.2)-(16.4)$; if $n=2$, only $\iota_{a, b}^{-}$is defined and, from (iii) above, $\iota_{a, b}^{-}=4 \theta_{a, b}-\varepsilon^{2}(a, b) \beta$.) Now (16.5), (16.3) and (16.7) give $n^{2} \Omega \theta_{a, b}=\tau_{e}-2 \mu_{a, b}+2 \varepsilon^{2}(a, b) \beta / n$ and $\Omega \mu_{a, b}=-2 \theta_{a, b}$, so that

$$
\begin{aligned}
& \Omega \iota_{a, b}^{ \pm}= \pm 2 \iota_{a, b}^{ \pm} / n, \quad \Omega \tau_{e}=\tau_{e}, \quad \Omega \beta=2 \beta \text { for } n \geq 3, \\
& \Omega \iota_{a, b}^{-}=-\iota_{a, b}^{-} \text {and } \Omega \beta=2 \beta \text { for } n=2 .
\end{aligned}
$$

The elements $\theta_{a, b}$ span $\mathcal{T}=\left[\mathfrak{g}^{*}\right]^{\odot 2}$, since $\theta_{a, b}$ is a multiple of the symmetric product $(a, \cdot) \odot(b, \cdot)$. As $2 n \theta_{a, b}=\iota_{a, b}^{+}+\iota_{a, b}^{-}+2 n\left(n^{2}-4\right)^{-1} \tau_{e}+2\left(n^{2}-1\right)^{-1} \varepsilon^{2}(a, b) \beta$ (if $n \geq 3$ ) or $4 \theta_{a, b}=\iota_{a, b}^{-}+\varepsilon^{2}(a, b) \beta$ (if $n=2$ ), (17.1) yields

$$
\begin{aligned}
& \mathcal{T}=\mathcal{V}_{+}+\mathcal{V}_{-}+\mathcal{V}+\mathbb{F} \beta \quad \text { for } n \geq 3, \quad \mathcal{T}=\mathcal{V}_{-}+\mathbb{F} \beta \text { for } n=2, \\
& \mathcal{V}_{ \pm} \subset \operatorname{Ker}(\Omega \mp 2 \operatorname{Id} / n), \quad \mathcal{V} \subset \operatorname{Ker}(\Omega-\mathrm{Id}), \quad \mathbb{F} \beta \subset \operatorname{Ker}(\Omega-2 \mathrm{Id}),
\end{aligned}
$$

where $\mathcal{V}_{ \pm}$(or, $\mathcal{V}$ ) is the subspace of $\mathcal{T}$ spanned by all $\iota_{a, b}^{ \pm}$(or, respectively, by all $\left.\tau_{a}\right)$. Since the eigenvalues in question are mutually distinct, $\Omega$ is diagonalizable and the above inclusions are equalities. Now (d) - (f) are obvious from (ii) above 
and the second part of (3.6), while $\operatorname{tr} \Omega=1-n^{2}$, cf. (8.9.iii) and (14.1), which uniquely determines the correct multiplicities (with $d^{-}=0$ for $n=3$ ).

Remark 17.2. Lemmas 17.1 and 13.1(b)-(c) easily imply that, for the underlying real Lie algebra $\mathfrak{g}_{\mathbb{R}}$ of $\mathfrak{g}=\mathfrak{s l}(n, \mathbb{C})$, the operator $\Omega_{\mathbb{R}}$ (notation of $(13.2)$ ) has the eigenvalues listed in (a) - (c) above, with twice the multiplicities of (a) - (c), plus the eigenvalue 0 with the multiplicity $\left(n^{2}-1\right)^{2}$. By Lemma 13.1(a)-(c), the eigenspaces for the eigenvalues 2 and 1 are $\mathbb{R}$-isomorphic images of those in (e) and (f) above under the operator $\sigma \mapsto \operatorname{Re} \sigma$.

Remark 17.3. Let $(\mathfrak{g}, \mathbb{F}, \varepsilon)$ be one of the triples (16.1), with $n=l+j \geq 3$. Then, by (12.3) and Lemma 17.1(d), the composite operator

$$
a \mapsto \tau_{a}=\tau \mapsto \mathrm{D} \tau \text { is a linear isomorphism } \mathfrak{g} \rightarrow \operatorname{Ker}(\Omega-\mathrm{Id}) \rightarrow \operatorname{Ker} \Delta .
$$

This is also true for $\mathfrak{g}=\mathfrak{s l}(n, \mathbb{C})$ treated as a real Lie algebra $\mathfrak{g}_{\mathbb{R}}$, with $\mathrm{D} \tau_{a}$ denoting the D-gradient, in the complex Lie algebra $\mathfrak{g}$, of the (complex-bilinear) symmetric tensor $\tau_{a}$. In fact, Ker $\Delta_{\mathbb{R}}=\operatorname{Ker} \Delta$ by Remark 13.3 (vi).

Remark 17.4. The surjective operator $\mathcal{S} \rightarrow \mathfrak{g}$, mentioned immediately after (2.15), is obtained as the composite of the projection $\Pi: \mathcal{S} \rightarrow \operatorname{Ker} \Delta$ in (12.6.b) followed by the inverse $\operatorname{Ker} \Delta \rightarrow \mathfrak{g}$ of the isomorphism in Remark 17.3.

\section{Multiplication tables}

In this section $(\mathfrak{g}, \mathbb{F}, \varepsilon)$ is always one of the triples (16.1), with $n=l+j \geq 3$, and $a$ denotes an arbitrary element of $\mathfrak{g}$. We use the notation of $(16.2)-(16.4)$.

For $\circledast$ and $\{\cdot\}$ defined in the lines following Lemma 10.1 and in (9.4),

$$
\begin{array}{ll}
\tau_{a} \circledast \tau_{a}=\tau_{c}+2\left(\xi \beta+\mu_{a}-2 \theta_{a}\right), & \theta_{a} \circledast \theta_{a}=\xi \theta_{a}, \\
\mu_{a} \circledast \mu_{a}=\xi \tau_{c}+\xi^{2} \beta+\mu_{c}-\theta_{c}, & \tau_{a} \circledast \theta_{a}=2 \theta_{a, c}, \\
\tau_{a} \circledast \mu_{a}=\xi \tau_{a}+2 \mu_{a, c}-2 \theta_{a, c}, & \theta_{a} \circledast \mu_{a}=\theta_{a, d}, \\
\beta \circledast \tau_{a}=\tau_{a}, \quad \beta \circledast \theta_{a}=\theta_{a}, & \beta \circledast \mu_{a}=\mu_{a},
\end{array}
$$

as one easily verifies with the aid of (16.2) - (16.4) and (16.6). Also,

$$
\begin{array}{ll}
\left\{\tau_{a} \cdot \tau_{a}\right\}=\xi \beta+\mu_{a}-2 \theta_{a}, & \left\{\mu_{a} \cdot \mu_{a}\right\}=\xi \mu_{a}-\theta_{c}, \\
2\left\{\tau_{a} \cdot \mu_{a}\right\}=\xi \tau_{a}-4 \theta_{a, c}, & \left\{\theta_{a} \cdot \theta_{a}\right\}=0, \\
2 n^{2}\left\{\tau_{a} \cdot \theta_{a}\right\}=\tau_{d}-\xi \tau_{a}-2 \mu_{a, c}+2 \varepsilon^{2}(a, c) \beta / n, & n^{2}\left\{\theta_{a} \cdot \mu_{a}\right\}=\mu_{a, d}-\xi \tau_{c}-\xi^{2} \beta-\mu_{c}+\varepsilon^{2}(a, c) \tau_{a} /(2 n) .
\end{array}
$$

If $\mathfrak{g}=\mathfrak{s l}(n, \mathbb{F}),(18.2)$ is immediate from (16.2) - (16.4) and (16.6). Specifically, we use the three steps of Remark 15.2 and Lemma 15.3. Remark 16.1 and Lemma 14.3(iii) now show that (18.2) holds for the remaining triples $(\mathfrak{s u}(l, j), \mathbb{R}, \mathrm{i})$ and $(\mathfrak{s l}(n / 2, \mathbb{H}), \mathbb{R}, 1)$ in $(16.1)$ as well.

Furthermore, (16.4) and (8.7.a) trivially imply that, for all $v \in \mathfrak{g}$,

$$
\left[\mathrm{D} \tau_{a}\right]_{v} v=\varepsilon\left[a, v^{2}\right], \quad n\left[\mathrm{D} \theta_{a}\right]_{v} v=\varepsilon^{2}[(a, v) a, v], \quad\left[\mathrm{D} \mu_{a}\right]_{v} v=\varepsilon^{2}[a, v a v]
$$


From (18.3), (16.4), (8.7.b) and (16.6.i) one in turn obtains

$$
\begin{aligned}
& \tau_{a}\left(\mathrm{D} \tau_{a}\right)=\mathrm{D} \tau_{c}, \quad \theta_{a}\left(\mathrm{D} \tau_{a}\right)=\theta_{a}\left(\mathrm{D} \theta_{a}\right)=\theta_{a}\left(\mathrm{D} \mu_{a}\right)=0, \\
& n\left[\tau_{a}\left(\mathrm{D} \theta_{a}\right)\right]_{v} v=\varepsilon^{2}[(a, v) c, v], \quad\left[\tau_{a}\left(\mathrm{D} \mu_{a}\right)\right]_{v} v=\varepsilon^{2}[c, v a v], \\
& {\left[\mu_{a}\left(\mathrm{D} \tau_{a}\right)+\mathrm{D}\left(\xi \tau_{a}\right)\right]_{v} v=\varepsilon^{2}\left(c v^{2} a-a v^{2} c\right)} \\
& n\left[\mu_{a}\left(\mathrm{D} \theta_{a}\right)+\mathrm{D}\left(\xi \theta_{a}\right)\right]_{v} v=\varepsilon^{3}(a, v)(c v a-a v c), \\
& {\left[\mu_{a}\left(\mathrm{D} \mu_{a}\right)+\mathrm{D}\left(\xi \mu_{a}\right)\right]_{v} v=\varepsilon^{3}(\text { cvava }- \text { avavc }) .}
\end{aligned}
$$

Lemma 18.1. For $(\mathfrak{g}, \mathbb{F}, \varepsilon)$ as in (16.1), $n=l+j \geq 3$, setting $\chi_{a}=2 \mu_{a}-n^{2} \theta_{a}+2 \xi \beta+$ $\left(n^{2}+4\right)\left(n^{2}-4\right)^{-1} \tau_{c}$ and $\phi_{a}=2 n^{2} \theta_{a}-n^{2} \mu_{a}-4 \xi \beta-4 n^{2}\left(n^{2}-4\right)^{-1} \tau_{c}$, in the notation of (8.8) and (16.2) - (16.4), we have, if $x, y, z \in \mathbb{F}$ and $\lambda=x \tau_{a}+n^{2} y \theta_{a}+z \mu_{a}$,

(i) $\left(n^{2}-4\right) \theta_{a}=\tau_{c}+(\Omega-\mathrm{Id}) \chi_{a}$ and $\left(n^{2}-4\right) \mu_{a}=-2 \tau_{c}+(\Omega-\mathrm{Id}) \phi_{a}$,

(ii) $\chi_{a}, \phi_{a} \in(\Omega-\mathrm{Id})(\mathcal{T})$,

(iii) $\Pi \tau_{a}=\tau_{a}, \quad\left(n^{2}-4\right) \Pi \theta_{a}=\tau_{c}, \quad\left(n^{2}-4\right) \Pi \mu_{a}=-2 \tau_{c}, \quad \Pi \beta=0$,

(iv) $\left(n^{2}-4\right)\left(\Pi \lambda-\tau_{a}\right)=\left(n^{2}-4\right)(x-1) \tau_{a}+\left(n^{2} y-2 z\right) \tau_{c}$,

$\Pi$ being the direct-sum projection in (12.6.a), well-defined in view of Lemma 17.1(f).

Proof. Assertion (i) is a trivial consequence of (16.7), while (iii) is immediate from (i) and (16.7), as $\Omega: \mathcal{T} \rightarrow \mathcal{T}$ is self-adjoint (Lemma 11.2(ii)). Now (iv) is obvious, and (iii) gives $\Pi \chi_{a}=\Pi \phi_{a}=0$, proving (ii).

\section{Some relevant algebraic sets in the nine-dimensional space}

Equations (19.1) - (19.2) discussed below, depending on an integer parameter $n$, are quite important in our argument. Namely, as we will show in the proof of Lemma 25.1, for the Lie algebras $\mathfrak{g}$ appearing in (16.1) with $n=l+j \geq 3$, any real-analytic curve of weakly-Einstein connections emanating from the standard connection D is naturally mapped into the solution set of (19.1) - (19.2) in $\mathbb{F}^{9}$, in such a way that the image of D is the point (19.3). The rationality conclusion of Lemma 24.1 then implies that the latter mapping must be constant. This means that the real-analytic curves in question all lie in a specific family $\mathcal{C}$ of Einstein connections, described by formula (22.3) in Section 22 .

Lemma 19.1. For $\mathbb{F}=\mathbb{R}$ or $\mathbb{F}=\mathbb{C}$ and any integer $n \geq 3$, the conditions

$$
\begin{aligned}
\text { i) } & f-\left(2 y+x^{2}+\xi z^{2}\right) \xi=0, \\
\text { ii) } & U+(y+x p) h=0 \\
\text { iii) } & \left(n^{2}-4\right)(x-1)+\left(n^{2} y-2 z\right) h=0, \\
\text { iv) } & V-n^{2} y-2 z=0 \\
\text { v) } & W-2 y-z=0 \\
\text { vi) } & p+x-2 \xi x z-h \xi z^{2}-h y=0, \\
\text { vii) } & q+n^{2}(L+2) y-V+2 z=0, \\
\text { viii) } & r+(L+2) z-W+2 y=0
\end{aligned}
$$


imposed on the nonuple $(\xi, f, h, x, y, z, p, q, r) \in \mathbb{F}^{9}$, where we have set

$$
\begin{aligned}
& L=f+h p-\xi r, \\
& U=2 \xi x z+h \xi z^{2}-\xi x r+x f, \\
& V=n^{2} L y+2 n^{2}\left(2 \xi z+h x+h^{2} z\right) y \\
& \quad+n^{4} \xi y^{2}-2 x^{2}+2(\xi z-h x) z, \\
& W=L z+x^{2}+\xi z^{2}+h^{2} z^{2}+2 h x z,
\end{aligned}
$$

constitute a system of eight polynomial equations in nine unknowns.

(a) A solution of (19.1), for which $(L, U, V, W)=(0,0,-2,1)$, is given by

$$
\xi=h=f=0, \quad x=-p=1, \quad q=-n^{2} y=\frac{4 n^{2}}{n^{2}-4}, \quad z=-r=\frac{n^{2}+4}{n^{2}-4} .
$$

(b) Solutions of (19.1) lying near (19.3) form the graph of an $\mathbb{F}$-analytic curve

$$
\xi \mapsto(f, h, x, y, z, p, q, r) \in \mathbb{F}^{8}, \quad \text { with } \mathrm{d} h / \mathrm{d} \xi=4 \text { at } \xi=0,
$$

where $\xi$ ranges over a neighborhood of 0 in $\mathbb{F}$.

Proof. First, (a) is obvious. Secondly, one easily verifies that

$$
\left[\begin{array}{cccccccc}
1 & 0 & 0 & 0 & 0 & 0 & 0 & 0 \\
* & n^{2} /\left(4-n^{2}\right) & 0 & 0 & 0 & 0 & 0 & 0 \\
* & * & n^{2}-4 & 0 & 0 & 0 & 0 & 0 \\
* & * & * & -n^{2} & -2 & 0 & 0 & 0 \\
* & * & * & -2 & -1 & 0 & 0 & 0 \\
* & * & * & * & * & 1 & 0 & 0 \\
* & * & * & * & * & * & 1 & 0 \\
* & * & * & * & * & * & * & 1
\end{array}\right]
$$

is the Jacobian matrix at the point (19.3) of the mapping $\mathbb{F}^{8} \rightarrow \mathbb{F}^{8}$ sending $(f, h, x, y, z, p, q, r)$ to the octuple formed by the left-hand sides in (19.1), in which $\xi$ has been replaced by 0 . (Each row of (19.5) thus represents the differential of the corresponding left-hand side written as a combination of $\mathrm{d} f, \mathrm{~d} h, \mathrm{~d} x, \mathrm{~d} y, \mathrm{~d} z, \mathrm{~d} p, \mathrm{~d} q$ and $\mathrm{d} r$, while asterisks stand for various irrelevant entries.) Replacing the fourth row in (19.5) by the fourth row minus twice the fifth row, we obtain a triangular matrix with nonzero diagonal entries, so that (19.5) is nonsigular and the existence of the curve required in (b), except for the specific value of $\mathrm{d} h / \mathrm{d} \xi$, is immediate from the implicit mapping theorem.

To evaluate $\mathrm{d} h / \mathrm{d} \xi$ at $\xi=0$, we may treat $f, h, x, y, z, p, q$ and $r$, restricted to the graph of the curve in (19.4), as functions of $\xi$. Applying $(,)^{\prime}=\mathrm{d} / \mathrm{d} \xi$ to (19.1.i), (19.1.ii) and the second line in (19.2), one sees that, at the point $(\xi, f, h, x, y, z, p, q, r)$ given by $(19.3), f^{\prime}=2 y+1, U^{\prime}=(1-y) h^{\prime}$, and $U^{\prime}=$ $2 z-r+f^{\prime}$. (We have used the fact that $\xi=h=f=0$ and $x=-p=1$.) Thus, $(1-y) h^{\prime}=U^{\prime}=2 z-r+f^{\prime}=2 z-r+2 y+1$, and so $h^{\prime}=4$. 
Remark 19.2. For later reference, note that, by (19.1.iv) - (19.1.v),

$V-2 W=\left(n^{2}-4\right) y, n^{2} W-2 V=\left(n^{2}-4\right) z, \quad\left(n^{2}+4\right) V-4 n^{2} W=\left(n^{2}-4\right)\left(n^{2} y-2 z\right)$.

\section{Negligible polynomials}

Given one of the triples (16.1), for $n=l+j \geq 3$, and a vector space $\mathcal{V}$ with $\operatorname{dim}_{\mathbb{F}} \mathcal{V}<\infty$, we define a $\mathcal{V}$-valued negligible polynomial function of the variables

$$
a, b \in \mathfrak{g} \text { and }(\xi, f, h, x, y, z, p, q, r) \in \mathbb{F}^{9}
$$

to be any mapping $\mathfrak{g} \times \mathfrak{g} \times \mathbb{F}^{9} \rightarrow \mathcal{V}$ expressible as a sum of terms, each of which is multilinear in $(a, b, \ldots)$, or $(b, b, \ldots)$, or $(\xi, b, \ldots)$, or $(h, b, \ldots)$, with the dots standing for any finite number of arguments from the list $a, b, \xi, f, h, x, y, z, p, q, r$, possibly with repetitions. In the case of two mappings $E, \tilde{E}: \mathfrak{g} \times \mathfrak{g} \times \mathbb{F}^{9} \rightarrow \mathcal{V}$,

$$
\text { we write } E \approx \tilde{E} \text { when } E-\tilde{E} \text { is negligible. }
$$

Lemma 20.1. For $E, \tilde{E}: \mathfrak{g} \times \mathfrak{g} \times \mathbb{F}^{9} \rightarrow \mathcal{V}$ as above, suppose that $E \approx \tilde{E}$.

(i) We have $E=\tilde{E}$ at any $(a, b, \xi, f, h, x, y, z, p, q, r)$ with $b=0$,

(ii) Let $(a, b, \xi, f, h, x, y, z, p, q, r)$ be $C^{\infty}$ functions of a variable $t \in[0, \delta)$, where $\delta \in(0, \infty)$. If $a(0)=0$ as well as $\xi(0)=h(0)=0$ and $j^{k-1}[b]=0$ for an integer $k \geq 1$, in the notation of (3.9), then $j^{k}[E]=j^{k}[\tilde{E}]$.

Proof. This is obvious from the definition of negligibility and the Leibniz rule.

In our discussion, negligible polynomials arise as follows. Choosing $(\mathfrak{g}, \mathbb{F}, \varepsilon)$ to be one of the triples (16.1), with $n=l+j \geq 3$, and using the notation of (16.2) (16.4), we introduce the variables (20.1) consisting of an arbitrary element $a$ of $\mathfrak{g}$, the coefficients $f, x, y, z, p, q, r$ of two arbitrary linear combinations

$$
\text { i) } \lambda=x \tau_{a}+n^{2} y \theta_{a}+z \mu_{a}, \quad \text { ii) } \psi=p \tau_{a}+q \theta_{a}+r \mu_{a}+f \beta,
$$

the scalar $\xi=\varepsilon^{2}(a, a) / n$ as in (16.3), where $(a, a)=\operatorname{tr} a^{2}$, an additional scalar variable $h \in \mathbb{F}$, and $b \in \mathfrak{g}$ given by

$$
b=c-h a, \quad \text { so that } c=h a+b,
$$

where $c=\varepsilon\left(a^{2}\right)_{0}$ stands for the traceless part of $\varepsilon a^{2}$, as in (14.2.ii) and (16.3).

Expressions such as $\lambda$ and $\psi$ in (20.3), along with their $\Omega$-images, $\circledast$-products and $\{\cdot\}$-products (which we may evaluate using (16.7), (18.1) and (18.2)), then become functions of the variables (20.1), and so do $c$ and $d$ in (16.3). Due to functional-dependence relations among our variables (20.1), there is a builtin ambiguity of such a representation, which we do not attempt to remove. For instance, one may treat $(a, a)$ as a function of $a$ alone, or write it as $n \varepsilon^{-2} \xi$. Similarly, $c=\varepsilon\left(a^{2}\right)_{0}$ equals both $\varepsilon a^{2}-\varepsilon^{-1} \xi$ and $h a+b$, cf. (16.6.i) and (20.4). 
With $\approx$ and $\lambda$ as in (20.2) and (20.3.i), we now have

i) $\tau_{c} \circledast \lambda \approx h \tau_{a} \circledast \lambda, \quad h \tau_{c} \approx h^{2} \tau_{a}, \quad \xi \tau_{c} \approx \xi h \tau_{a}, \quad \varepsilon^{2}(a, c) / n \approx \xi h$,

ii) $\mu_{c} \approx h^{2} \mu_{a}, \quad \theta_{c} \approx h^{2} \theta_{a}, \quad \theta_{a, c} \approx h \theta_{a}, \quad \mu_{a, c} \approx h \mu_{a}$.

Namely, (20.5.i) is obvious as $\tau_{c}-h \tau_{a}=\tau_{b}$ by (20.4), and $(a, c)-h(a, a)=(a, b)$, while (20.5.ii) follows from (20.4) and (16.3). Furthermore,

$$
d \approx\left(h^{2}+\xi\right) a .
$$

In fact, (16.6.i) implies that $\varepsilon^{2} a^{3}=\varepsilon\left(\varepsilon a^{2}\right) a=\varepsilon c a+\xi a$ and, as a consequence of (16.6.ii), $d=\varepsilon c a+\xi a-\varepsilon(a, c) / n$. Replacing $c$ here by $h a+b$, and the resulting occurrence of $\varepsilon a^{2}$ by $h a+b+\varepsilon^{-1} \xi$, which is allowed due to (16.6.i) and (20.4), we see that $d-\left(h^{2}+\xi\right) a=\varepsilon b a+h b-\varepsilon(a, b) / n$ due to the equality $\xi=\varepsilon^{2}(a, a) / n$, which yields (20.6). Now (20.6) gives

$$
\tau_{d} \approx\left(h^{2}+\xi\right) \tau_{a}, \quad \theta_{a, d} \approx\left(h^{2}+\xi\right) \theta_{a}, \quad \mu_{a, d} \approx\left(h^{2}+\xi\right) \mu_{a}
$$

Lemma 20.2. If $(\mathfrak{g}, \mathbb{F}, \varepsilon)$ is one of the triples (16.1), and $n=l+j \geq 3$, then

$$
\begin{array}{ll}
\tau_{a} \circledast \tau_{a}=\tau_{c}+2\left(\xi \beta+\mu_{a}-2 \theta_{a}\right), & \theta_{a} \circledast \theta_{a}=\xi \theta_{a}, \\
\mu_{a} \circledast \mu_{a} \approx \xi h \tau_{a}+\xi^{2} \beta+h^{2}\left(\mu_{a}-\theta_{a}\right), & \tau_{a} \circledast \theta_{a} \approx 2 h \theta_{a}, \\
\tau_{a} \circledast \mu_{a} \approx \xi \tau_{a}+2 h\left(\mu_{a}-\theta_{a}\right), & \theta_{a} \circledast \mu_{a} \approx\left(h^{2}+\xi\right) \theta_{a}, \\
\beta \circledast \tau_{a}=\tau_{a}, \quad \beta \circledast \theta_{a}=\theta_{a}, & \beta \circledast \mu_{a}=\mu_{a},
\end{array}
$$

with the dependence on the variables (20.1) described above. Similarly,

$$
\begin{array}{ll}
\left\{\tau_{a} \cdot \tau_{a}\right\}=\xi \beta+\mu_{a}-2 \theta_{a}, & \left\{\mu_{a} \cdot \mu_{a}\right\} \approx \xi \mu_{a}-h^{2} \theta_{a}, \\
2\left\{\tau_{a} \cdot \mu_{a}\right\} \approx \xi \tau_{a}-4 h \theta_{a}, & \left\{\theta_{a} \cdot \theta_{a}\right\}=0, \\
2 n^{2}\left\{\tau_{a} \cdot \theta_{a}\right\} \approx h^{2} \tau_{a}-2 h \mu_{a}+2 \xi h \beta, & \\
n^{2}\left\{\theta_{a} \cdot \mu_{a}\right\} \approx \xi \mu_{a}-\xi^{2} \beta-\xi h \tau_{a} / 2 .
\end{array}
$$

Proof. This is immediate from (20.5) - (20.7) combined with (18.1) - (18.2).

Lemma 20.3. Under the hypotheses of Lemma 20.2, for $L, U, V, W$ given by (19.2),

$4\{(\mathrm{D} \lambda) \cdot(\mathrm{D} \lambda)\} \approx(U-L x+h x p) \tau_{a}+\left(V-n^{2} L y\right) \theta_{a}+(W-L z) \mu_{a}+\left(x^{2}+\xi z^{2}\right) \xi \beta$, where $\lambda=x \tau_{a}+n^{2} y \theta_{a}+z \mu_{a}$, as in (20.3).

Proof. The second part of Lemma 20.2 yields

$$
\begin{aligned}
\{\lambda \cdot \lambda\} & \approx\left(h^{2} x y+\xi x z-h \xi y z\right) \tau_{a}-\left[2 x^{2}+(h z+4 x) h z\right] \theta_{a} \\
& +\left(x^{2}+\xi z^{2}-2 h x y+2 \xi y z\right) \mu_{a}+\left(x^{2}+2 h x y-2 \xi y z\right) \xi \beta .
\end{aligned}
$$

By $(16.8),(\operatorname{Id}-\Omega) \lambda=\left(n^{2} y+2 z\right) \theta_{a}+(2 y+z) \mu_{a}-2 \xi y \beta-y \tau_{c}$. Replacing $y \tau_{c}$ with $h y \tau_{a}$, cf. (20.5.i), we get, from (20.5.i) and the first part of Lemma 20.2,

$$
\begin{aligned}
{[(\operatorname{Id}-\Omega) \lambda] \circledast \lambda } & \approx\left(\xi x z+h \xi y z-h^{2} x y+h \xi z^{2}\right) \tau_{a} \\
& +\left[V-n^{2} L y+2 x^{2}+(h z+4 x) h z\right] \theta_{a} \\
& +\left[h^{2} z^{2}+2(h x y+h x z-\xi y z)\right] \mu_{a}+\left(\xi z^{2}+2 \xi y z-2 h x y\right) \xi \beta .
\end{aligned}
$$

In view of $(10.5 . \mathrm{c}), 4\{(\mathrm{D} \lambda) \cdot(\mathrm{D} \lambda)\}=\{\lambda \cdot \lambda\}+[(\mathrm{Id}-\Omega) \lambda] \circledast \lambda$, as required. 


\section{The first crucial step in the argument}

The following result will be used both to verify that the family $\mathcal{C}$ defined by formula (22.3) of Section 22 actually consists of Einstein connections, and to prove, later in Section 25, that $\mathcal{C}$ contains every real-analytic curve of weakly-Einstein connections emanating from D.

Theorem 21.1. With the assumptions and notations as in Lemmas 20.2 and 20.3,

$$
4 \mathrm{D}\{(\mathrm{D} \lambda) \cdot(\mathrm{D} \lambda)\}+(\mathrm{D} \lambda) \psi \approx \mathrm{D}\left(U \tau_{a}+h x p \tau_{a}+V \theta_{a}+W \mu_{a}+x p \tau_{b}\right),
$$

while for $\mathrm{M}=\Delta(\mathrm{D} \lambda)+4 \mathrm{D}\{(\mathrm{D} \lambda) \cdot(\mathrm{D} \lambda)\}+(\mathrm{D} \lambda) \psi$ we have

$$
\begin{aligned}
\mathrm{M} & \approx \mathrm{D}\left[U \tau_{a}+(y+x p) h \tau_{a}+(y+x p) \tau_{b}\right] \\
& +\mathrm{D}\left[\left(V-n^{2} y-2 z\right) \theta_{a}+(W-2 y-z) \mu_{a}\right],
\end{aligned}
$$

and, setting $\zeta=\psi-8\{\mathrm{D} \cdot(\mathrm{D} \lambda)\}-4\{(\mathrm{D} \lambda) \cdot(\mathrm{D} \lambda)\}$, we obtain

$$
\begin{aligned}
\zeta & \approx\left(p+x-2 \xi x z-h \xi z^{2}-h y\right) \tau_{a}+\left[q+n^{2}(L+2) y-V+2 z\right] \theta_{a} \\
& +[r+(L+2) z-W+2 y] \mu_{a}+\left[f-\left(2 y+x^{2}+\xi z^{2}\right) \xi\right] \beta-y \tau_{b} .
\end{aligned}
$$

Proof. Replacing $c$ in (18.4) with $h a+b$ (see (20.4)) we have, from (18.3),

$$
\begin{aligned}
& \tau_{a}\left(\mathrm{D} \tau_{a}\right)=\mathrm{D}\left(h \tau_{a}\right)+\mathrm{D} \tau_{b}, \quad \tau_{a}\left(\mathrm{D} \theta_{a}\right) \approx \mathrm{D}\left(h \theta_{a}\right), \quad \tau_{a}\left(\mathrm{D} \mu_{a}\right) \approx \mathrm{D}\left(h \mu_{a}\right), \\
& \theta_{a}\left(\mathrm{D} \tau_{a}\right)=\theta_{a}\left(\mathrm{D} \theta_{a}\right)=\theta_{a}\left(\mathrm{D} \mu_{a}\right)=0, \\
& \mu_{a}\left(\mathrm{D} \tau_{a}\right) \approx-\mathrm{D}\left(\xi \tau_{a}\right), \quad \mu_{a}\left(\mathrm{D} \theta_{a}\right) \approx-\mathrm{D}\left(\xi \theta_{a}\right), \quad \mu_{a}\left(\mathrm{D} \mu_{a}\right) \approx-\mathrm{D}\left(\xi \mu_{a}\right), \\
& \beta\left(\mathrm{D} \tau_{a}\right)=\mathrm{D} \tau_{a}, \quad \beta\left(\mathrm{D} \theta_{a}\right)=\mathrm{D} \theta_{a}, \quad \beta\left(\mathrm{D} \mu_{a}\right)=\mathrm{D} \mu_{a},
\end{aligned}
$$

the last line being obvious from (8.4) since $\sigma=\beta$ corresponds via (7.3) to $\Sigma=$ Id. If one were to treat $\approx$ as equality ignore the "correction term" $\mathrm{D} \tau_{b}$, the D-images of $\tau_{a}, \theta_{a}, \mu_{a}$, and of their combination $\lambda$, would lie in the eigenspace, for the eigenvalue $h, 0,-\xi$ or 1 , of the operator $\mathcal{S} \rightarrow \mathcal{S}$ sending $\mathrm{S}$, respectively, to $\tau_{a} \mathrm{~S}, \theta_{a} \mathrm{~S}, \mu_{a} \mathrm{~S}$ or $\beta \mathrm{S}$. Thus, up to such an equivalence, $\mathrm{D} \lambda$ lies in the eigenspace of the operator $\mathrm{S} \mapsto \psi \mathrm{S}=\left(p \tau_{a}+q \theta_{a}+r \mu_{a}+f \beta\right) \mathrm{S}$ for the combined eigenvalue $L$ given by (19.2). Restoring the correction term, we now have $\psi(\mathrm{D} \lambda) \approx \mathrm{D}\left(L \lambda+x p \tau_{b}\right)$, and so

$$
(\mathrm{D} \lambda) \psi \approx \mathrm{D}\left(L \lambda+x p \tau_{b}\right)
$$

since Lemma 10.1 and its proof remain valid for $\approx$ equivalences instead of equalities. Hence (21.1) is immediate from Lemma 20.3, with $\mathrm{D} \beta=0$ by (9.6.c). Relation (21.2) is in turn a trivial consequence of (21.1) and formula (12.1) for $\sigma=\lambda$, combined with (16.8) and (20.4). Finally, as (10.5.b) yields $-8\{\mathrm{D} \cdot(\mathrm{D} \lambda)\}=$ $\lambda+(\operatorname{Id}-\Omega) \lambda,(21.3)$ easily follows from (20.3), (20.4), (16.8) and Lemma 20.3.

Remark 21.2. The polynomial mappings $\boldsymbol{J}, \boldsymbol{M}$ of Section 2 are defined as follows. For $\mathbf{v}=(\xi, f, h, x, y, z, p, q, r) \in \mathbb{F}^{9}$, the first component of $\mathbf{J}(\mathbf{v})$ is 0 , and the other eight components are the left-hand sides of (i), (iii), (ii) and (iv) - (viii) in (19.1), with (19.2), while $\boldsymbol{M}(\mathbf{v})=(0,0,0, y+x p, 0,0,-y, 0,0)$. 


\section{The main results}

Given a real (or, complex) Lie group $\mathrm{G}$ with the Lie algebra $\mathfrak{g}$, we again refer to the Levi-Civita connections of left-invariant Einstein metrics on G as Einstein connections in $\mathfrak{g}$. The metrics themselves are pseudo-Riemannian or, respectively, holomorphic; $\beta$ and $\mathrm{D}=[] /$,2 stand for the Killing form, with (7.2), and its LeviCivita connection; and $\mathrm{D} \lambda$ is defined as in (8.3) or, equivalently, (8.7.a). The more general classes of unimodular torsion-free connections with parallel Ricci tensor, and weakly-Einstein connections, were introduced in Section 11.

Whenever $(\mathfrak{g}, \mathbb{F}, \varepsilon)$ is one of the triples (16.1), with $n=l+j$, while $\xi, h \in \mathbb{F}$, the scalar $\varepsilon^{-1} \xi$ denotes a multiple of Id, and $a \in \mathfrak{g}$, one clearly has

$$
\varepsilon a^{2}=h a+\varepsilon^{-1} \xi
$$

if and only if $\xi=\varepsilon^{2}(a, a) / n$ and $c=h a$, where $c=\varepsilon\left(a^{2}\right)_{0}$. (Notation of (16.3).)

Theorem 22.1. Let $(\mathfrak{g}, \mathbb{F}, \varepsilon)$ be one of (16.1), $n=l+j \geq 3$. If $\lambda$, $\psi$ are given by (20.3) for $\xi, f, h, x, y, z, p, q, r \in \mathbb{F}$ satisfying (19.1) with (19.2), except (19.1.iii), while $a \in \mathfrak{g}$ and (22.1) holds, then $\nabla=\mathrm{D}+\mathrm{D} \lambda$ is a weakly-Einstein, as well as unimodular, torsion-free connection in $\mathfrak{g}$ with the $\nabla$-parallel Ricci tensor $\rho^{\nabla}=-(\beta+\psi) / 4$.

Proof. By (20.4), the assumption (22.1), that is, $c=h a$, gives $b=0$, and so, in view of Lemma 20.1(i), $\approx$ equivalences are actually equalities. Applied to (21.2) and (21.3), this yields $\mathrm{M}=0$ and $\zeta=0$, since the right-hand sides of (21.2) and (21.3) vanish as a consequence of (19.1) (while (19.1.iii) is not used). Combined with the definitions of $\mathrm{M}$ and $\zeta$ in Theorem 21.1, the relations $\mathrm{M}=0$ and $\zeta=0$ state that, by (8.6), condition (a) of Lemma 11.1 holds for $\mathrm{S}=\mathrm{D} \lambda$ and $\sigma=\psi$. Since (b) in Lemma 11.1 is equivalent to (a), our claim follows, cf. (11.1).

Under the assumption $c=h a$ (that is, (22.1)) made in Theorem 22.1,

(19.1.iii) holds if and only if $\Pi \lambda=\tau_{a}$, where $\lambda=x \tau_{a}+n^{2} y \theta_{a}+z \mu_{a}$,

as one sees using Lemma 18.1(iv). The dependence of $\tau_{a}$ (or, $\theta_{a}$ and $\mu_{a}$ ) on $a$ is, by (16.4), homogeneous linear (or, respectively, quadratic). Consequently, $\lambda$ and $\psi$ in (20.3), along with $\nabla$ and $\rho^{\nabla}$, will remain unchanged if we replace $a$ with a nonzero multiple, and at the same time suitably rescale $f, x, y, z, p, q$ and $r$. Thus, (19.1.iii) is a normalizing condition, which can always be realized by rescaling, as long as $\Pi \lambda \neq 0$ (or, equivalently, $\left(n^{2}-4\right) x+\left(n^{2} y-2 z\right) h \neq 0$ ), while the system (19.1) without equation (19.1.iii) is rescaling-invariant.

In terms of the inclusions $\mathcal{E} \subset \mathcal{U} \subset \mathcal{W}$, cf. (11.1), all connections obtained in Theorem 22.1 lie in $\mathcal{U}$, and hence in $\mathcal{W}$ but, as shown below in Section 29, they need not be Einstein connections (elements of $\mathcal{E}$ ).

However, Einstein connections do arise in a special case of Theorem 22.1, which is the first part of the main result of this paper, stated as follows. 
Theorem 22.2. Suppose that $(\mathfrak{g}, \mathbb{F}, \varepsilon)$ is one of the triples (16.1):

$(\mathfrak{s l}(n, \mathbb{R}), \mathbb{R}, 1),(\mathfrak{s l}(n, \mathbb{C}), \mathbb{C}, 1),(\mathfrak{s l}(n, \mathbb{C}), \mathbb{C}, \mathrm{i}),(\mathfrak{s u}(l, j), \mathbb{R}, \mathrm{i}),(\mathfrak{s l}(n / 2, \mathbb{H}), \mathbb{R}, 1)$, with $n=l+j \geq 3$, and $\tau_{a}, \theta_{a}, \mu_{a}$ are defined by (16.4). Then the set

$$
\begin{aligned}
& \mathcal{C}=\mathrm{D}+\mathcal{L} \text { for } \mathcal{L}=\left\{\mathrm{D} \eta_{a}: a \in \mathfrak{g} \text { and } a^{2}=0\right\}, \\
& \text { where } \eta_{a}=\tau_{a}-\left(n^{2}-4\right)^{-1}\left[4 n^{2} \theta_{a}-\left(n^{2}+4\right) \mu_{a}\right],
\end{aligned}
$$

consists of Einstein connections in $\mathfrak{g}$. If $\beta$ denotes the Killing form, with (7.2),

(i) every $\nabla \in \mathcal{C}$ has the nondegenerate $\nabla$-parallel Ricci tensor $\rho^{\nabla}=\left(\eta_{a}-\beta\right) / 4$,

(ii) the assignment $a \mapsto \mathrm{D}+\mathrm{D} \eta_{a}$ maps $\mathcal{P}=\left\{a \in \mathfrak{g}: a^{2}=0\right\}$ bijectively onto $\mathcal{C}$,

(iii) the inverse of the bijection in (ii) is $\nabla \mapsto a$ for $a \in \mathfrak{g}$ uniquely characterized by $\Pi(\nabla-\mathrm{D})=\mathrm{D} \tau_{a}$, with $\Pi$ as in (12.6.b).

Finally, for the set $\mathcal{C}$ in (22.3), and $\mathcal{W}$ given by (11.1),

(iv) $\mathcal{C}$ is an algebraic set in $\mathcal{Y}=\left[\mathfrak{g}^{*}\right]^{\otimes 2} \otimes \mathfrak{g}$, and hence a closed subset of $\mathcal{Y}$,

(v) $\mathcal{C}$ is relatively open in the set $\mathcal{E}$ of all Einstein connections, and in $\mathcal{W}$,

(vi) $\mathcal{C}$ forms a connected component of both $\mathcal{E}$ and $\mathcal{W}$.

(vii) $\operatorname{dim}_{\mathbb{F}} \mathcal{C}=\left[n^{2} / 2\right]$ for $\mathfrak{g}=\mathfrak{s l}(n, \mathbb{F})$, while $\operatorname{dim}_{\mathbb{R}} \mathcal{C}=2 l j$ when $\mathfrak{g}=\mathfrak{s u}(l, j)$, and $\operatorname{dim}_{\mathbb{R}} \mathcal{C}=4\left[n^{2} / 8\right]$ if $\mathfrak{g}=\mathfrak{s l}(n / 2, \mathbb{H})$,

(viii) for the three choices of $\mathfrak{g}$ in (vii), $\mathcal{C}$ is a union of $[n / 2]+1$, or of $(j+1)(j+2) / 2$, or, respectively, of $[n / 4]+1$ adjoint-action orbits.

Explicitly, for $\nabla=\mathrm{D}+\mathrm{D} \eta_{a}$ and $v, w \in \mathfrak{g}$, we have

$2 \nabla_{v} w=[v, w]+\varepsilon\left[a, v w+w v+\mathrm{E}_{v}^{a} w\right], \quad$ where

$\left(n^{2}-4\right) \mathrm{E}_{v}^{a} w=\left(n^{2}+4\right) \varepsilon(v a w+w a v)-4 n \varepsilon(\operatorname{tr} a v) w-4 n \varepsilon(\operatorname{tr} a w) v$.

Theorem 22.3. A fixed positive-definite multiple of the Killing form is isolated in the set of suitably normalized left-invariant Riemannian Einstein metrics on $\mathrm{SU}(n), n \geq 3$.

Theorem 22.3 settles a rather narrow special case of Böhm, Wang and Ziller's Finiteness Conjecture [4, p. 683].

It is not known whether the Killing form of $\mathrm{SU}(n)$, for $n \geq 3$, represents an isolated point of the moduli space of all Riemannian Einstein metrics on $\mathrm{SU}(n)$ modulo diffeomorphisms and scaling. Cf. [10, Theorem 1.2].

Theorem 22.4. The conclusions of Theorem 22.2 remain valid for $\mathfrak{s l}(n, \mathbb{C})$ treated as a real Lie algebra, except that the the formula in (i) now reads $\rho^{\nabla}=2 \operatorname{Re}\left(\eta_{a}-\beta\right) / 4$.

In other words, $\mathcal{C}$ is a connected component of the set of all real Einstein connections in $\mathfrak{s l}(n, \mathbb{C})$, and similarly for weakly-Einstein connections. In particular, all weakly-Einstein connections in the underlying real Lie algebra of $\mathfrak{s l}(n, \mathbb{C})$, sufficiently close to $\mathrm{D}$, are necessarily holomorphic, that is, constitute $\mathbb{C}$-bilinear mappings $\mathfrak{g} \times \mathfrak{g} \rightarrow \mathfrak{g}$.

The conclusion in the final paragraph of the Introduction easily follows from Theorem 22.4. See also Remark 13.3.

The proof of Theorem 22.2 will be given later, in Sections 23 and 28. For proofs of Theorems 22.3 and 22.4, see Sections 26 and 28. 


\section{Proof of Theorem 22.2, first part}

In this section we verify all claims made in Theorem 22.2 except (v) - (viii).

The hypotheses of Theorem 22.1 are clearly satisfied by any $a \in \mathfrak{g}$ such that $a^{2}=0$ and the nonuple $(\xi, f, h, x, y, z, p, q, r)$ with (19.3). Since (20.3) then yields $\lambda=-\psi=\eta_{a}$, Theorem 22.1 implies that $\mathcal{C}$ with (22.3) consists of torsion-free connections $\nabla$ having the $\nabla$-parallel Ricci tensor given by the formula in (i).

As $a^{2}=0$ and $\xi=h=0,(16.3)$ and (20.4) yield $c=b=0$. According to Lemma 20.1(i), $\approx$ equivalences in the first part of Lemma 20.2 now become equalities: $\tau_{a} \circledast \tau_{a}=2\left(\mu_{a}-2 \theta_{a}\right)$ and $\theta_{a} \circledast \theta_{a}=\mu_{a} \circledast \mu_{a}=\tau_{a} \circledast \theta_{a}=\tau_{a} \circledast \mu_{a}=\theta_{a} \circledast \mu_{a}=0$. All three-factor $\circledast$-products of linear combinations of $\tau_{a}, \theta_{a}, \mu_{a}$ must therefore vanish. In particular, $\Sigma^{3}=0$ for the endomorphism $\Sigma$ of $\mathfrak{g}$ corresponding to $\sigma=\eta_{a}$ via (7.3), and so 0 is the only eigenvalue of $\Sigma$. Consequently, -1 is the only eigenvalue of $\Sigma-\mathrm{Id}$, and the Ricci tensor $\rho^{\nabla}=\left(\eta_{a}-\beta\right) / 4$ is nondegenerate, as required in (i). Since $\rho^{\nabla}$ is $\nabla$-parallel, $\nabla$ is its Levi-Civita connection (Remark 5.2). Thus, $\rho^{\nabla}$ is an Einstein metric, which shows that $\mathcal{C}$ consists of Einstein connections.

Let $\nabla \in \mathcal{C}$. Since (19.1.iii) holds, (22.2) yields $\Pi \eta_{a}=\tau_{a}$ and so, from Remark 12.2, $\Pi\left(\mathrm{D} \eta_{a}\right)=\mathrm{D} \tau_{a}$. (Again, as $\xi, f, h, x, y, z, p, q$ and $r$ are given by (19.3), $\lambda=x \tau_{a}+n^{2} y \theta_{a}+z \mu_{a}$ coincides with $\eta_{a}$.) This proves assertions (ii) (iii): $\nabla \in \mathcal{Y}=\left[\mathfrak{g}^{*}\right]^{\otimes 2} \otimes \mathfrak{g}$ is an element of $\mathcal{C}$ if and only if

$$
\nabla=\mathrm{D}+\mathrm{D} \eta_{a} \quad \text { and } \quad a^{2}=0
$$

where $a \in \mathfrak{g}$ is uniquely characterized by $\Pi(\nabla-\mathrm{D})=\mathrm{D} \tau_{a}$, that is, by being the image of $\Pi(\nabla-D)$ under the inverse of the isomorphism in Remark 17.3.

Now (iv) follows as well, since (23.1) is a system of (nonhomogeneous) quadratic equations imposed on $\nabla$.

Finally, the explicit expression (22.4) is immediate from (7.4), (18.3) combined with symmetry of $\mathrm{D} \eta_{a}$ (due to (8.3) applied to $\left.\nabla=\mathrm{D}\right)$, and (3.1).

\section{The rationality condition}

The use of Theorem 22.1 to construct examples of weakly-Einstein connections other than $\mathrm{D}$ requires not just finding $\xi, f, h, x, y, z, p, q, r \in \mathbb{F}$ that satisfy (19.1), but also realizing the assumption (22.1) with $a \neq 0$. This leads to the following additional restriction.

Lemma 24.1. If $(\mathfrak{g}, \mathbb{F}, \varepsilon)$ are as in (16.1) and $n=l+j \geq 3$, while $\xi, h \in \mathbb{F}$ and there exists $a \in \mathfrak{g} \backslash\{0\}$ satisfying (22.1), then

$$
n^{+} n^{-} h^{2}=\left(n^{+}-n^{-}\right)^{2} \xi \text { for some integers } n^{ \pm} \geq 1 \text { with } n^{+}+n^{-}=n .
$$

Proof. For a treated as a complex matrix, (22.1) gives $(\varepsilon a-h / 2)^{2}=\omega^{2}$, where $\omega$ is a complex square root of $\xi+h^{2} / 4$. We may assume that $\omega \neq 0$, since otherwise $\varepsilon a-h / 2$ is nilpotent, and therefore traceless, so that $h=0$, and $\xi=\varepsilon^{2}(a, a) / n=$ 
0 , which yields (24.1). Now $\mathbb{C}^{n}$ is the direct sum of $\operatorname{Ker}(\varepsilon a \mp \omega-h / 2)$, the eigenspaces of $a$. Their dimensions $n^{ \pm}$are positive, or else $a \neq 0$ would be a traceless multiple of Id. Also, $0=\operatorname{tr} \varepsilon a=n^{+}(\omega+h / 2)-n^{-}(\omega-h / 2)=\left(n^{+}-n^{-}\right) \omega+n h / 2$, that is, $\omega=n h /\left[2\left(n^{-}-n^{+}\right)\right]$. As $\omega^{2}=\xi+h^{2} / 4$, this gives $\xi+h^{2} / 4=\omega^{2}=$ $n^{2} h^{2} /\left[2\left(n^{+}-n^{-}\right)\right]^{2}$, completing the proof.

\section{Real-analytic curves through 0 in $\boldsymbol{H}^{-1}(0)$}

As before, in this section $(\mathfrak{g}, \mathbb{F}, \varepsilon)$ is one of the triples (16.1), $n=l+j \geq 3$, and $\mathcal{T}, \mathcal{S}$ are the spaces (7.1), while $\boldsymbol{H}: \mathcal{S} \rightarrow \mathcal{S}$ is given by (11.2.i). Recall that torsion-free connections $\nabla$ in $\mathfrak{g}$ form the affine space $\mathrm{D}+\mathcal{S}$, and so $\nabla=\mathrm{D}+\mathrm{S}$, where $\mathrm{S}: \mathfrak{g} \times \mathfrak{g} \rightarrow \mathfrak{g}$ is $\mathbb{F}$-bilinear and symmetric.

The following lemma provides the second crucial step in our argument: the conclusion that, for the sets $\mathcal{C}$ and $\mathcal{W}$ appearing in (22.3) and (11.1), $\mathcal{C}$ contains all real-analytic curves in $\mathcal{W}$, emanating from $\mathrm{D}$.

Lemma 25.1. Every real-analytic curve $[0, \delta) \ni t \mapsto \mathrm{D}+\mathrm{S}(t) \in \mathrm{D}+\mathcal{S}$, consisting of left-invariant torsion-free connections in $\mathfrak{g}$ with $\mathbf{H}(\mathrm{S}(t))=0$ and $\mathrm{S}(0)=0$, lies entirely in the set $\mathcal{C}=\mathrm{D}+\mathcal{L}$ of Einstein connections, defined by (22.3). This remains true for

$$
\text { the underlying real Lie algebra } \mathfrak{g}_{\mathbb{R}} \text { of } \mathfrak{g}=\mathfrak{s l}(n, \mathbb{C}) \text {, }
$$

with $\mathbb{C}$-bilinear $\mathrm{D} \eta_{a}$ in $(22.3)$, even though $\mathrm{S}(t)$ are only assumed $\mathbb{R}$-bilinear.

Proof. For such a curve $t \mapsto \mathrm{S}(t)$, let $\sigma=\sigma(t) \in \mathcal{T}$ and $a=a(t) \in \mathfrak{g}$ be defined by (11.3.b) and $\Pi \mathrm{S}=\mathrm{D} \tau_{a}$, where $\mathrm{S}=\mathrm{S}(t)$ and $\Pi$ is the projection (12.6.b). (See Remark 17.3.) Besides S, $\sigma$ and $a$, we introduce another curve parametrized by $t \in[0, \delta)$, namely, $c=c(t) \in \mathfrak{g}$, with (16.3), plus nine $\mathbb{F}$-valued real-analytic functions of $t$, which are $\xi=\varepsilon^{2}(a, a) / n$, for $(a, a)=\operatorname{tr} a^{2}$, and $f, h, x, y, z, p, q, r$, depending on $\xi$ (and hence on $t$, with smaller $\delta$ ) via (19.4). We also use $\lambda=\lambda(t)$ and $\psi=\psi(t)$ given by (20.3), with our $f, h, x, y, z, p, q, r$ and $a=a(t)$. For all $t$,

$$
\begin{aligned}
\text { i) } & -\Delta \mathrm{S}=4 \mathrm{D}\{\mathrm{S} \cdot \mathrm{S}\}+\mathrm{S} \sigma, \\
\text { ii) } & \sigma=8\{\mathrm{D} \cdot \mathrm{S}\}+4\{\mathrm{~S} \cdot \mathrm{S}\}, \\
\text { iii) } & \mathrm{S}-\mathrm{D} \lambda \in \Delta(\mathcal{S}) .
\end{aligned}
$$

In fact, as $\boldsymbol{H}(\mathrm{S}(t))=0$, (11.6) gives (25.2.i-ii). Our $\xi, f, h, x, y, z, p, q, r$ satisfy (19.1), including (19.1.iii), so that, from (22.2), $\Pi \lambda=\tau_{a}$, and hence $\Pi(\mathrm{D} \lambda)=\mathrm{D} \tau_{a}$ (see Remark 12.2). However, our choice of $a$ is characterized by $\Pi S=\mathrm{D} \tau_{a}$. Thus, $\Pi(\mathrm{S}-\mathrm{D} \lambda)=0$, which yields (25.2.iii).

We now proceed to show that, for $b=c-h a$, at every $t \in[0, \delta)$,

$$
\text { i) } b=0, \quad \text { ii) } \mathrm{S}=\mathrm{D} \lambda, \quad \text { iii) } \sigma=\psi \text {. }
$$

[In case (25.1), $\mathrm{S}=\mathrm{S}(t)$ and $\sigma$ are $\mathbb{R}$-bilinear, and (25.2) needs to be rewritten so as to use the notation of (13.2). However, $a \in \mathfrak{g}=\mathfrak{s l}(n, \mathbb{C})$, and $\xi$ (equal to $\left.\varepsilon^{2}(a, a) / n\right)$, as well as $f, h, x, y, z, p, q, r$ are complex numbers. Consequently, $\lambda, \psi$ 
are $\mathbb{C}$-bilinear (since so are their ingredients $\left.\tau_{a}, \theta_{a}, \mu_{a}, \beta\right)$, and (25.3.iii) must be replaced with $\sigma=2 \operatorname{Re} \psi$.]

To prove (25.3), we establish, by induction on $k \geq 0$, the equalities

$$
\text { i) } \mathrm{j}^{k}[b]=0, \quad \text { ii) } \mathrm{j}^{k}[\mathrm{~S}]=\mathrm{j}^{k}[\mathrm{D} \lambda], \quad \text { iii) } \mathrm{j}^{k}[\sigma]=\mathrm{j}^{k}[\psi]
$$

of $k$-jets at $t=0$ (notation of (3.9)). First, our "initial condition" $\mathrm{S}(0)=0$ and the above definitions of $a, c, \sigma, \lambda, \psi, \xi$ and $h$ give, by (19.3),

$$
\mathrm{S}(0)=0, \quad a(0)=c(0)=0, \quad \sigma(0)=\lambda(0)=\psi(0)=0, \quad \xi(0)=h(0)=0 .
$$

Thus, (25.4) holds for $k=0$. Assume now that $k \geq 1$ and

$$
\text { i) } \mathrm{j}^{k-1}[b]=0, \quad \text { ii) } \mathrm{j}^{k-1}[\mathrm{~S}]=\mathrm{j}^{k-1}[\mathrm{D} \lambda], \quad \text { iii) } \mathrm{j}^{k-1}[\sigma]=\mathrm{j}^{k-1}[\psi] \text {. }
$$

As $\mathrm{S}(0)=0$ and $\sigma(0)=0$ (see $(25.5))$, combining $(3.8)$, for $B(\mathrm{~S}, \tilde{\mathrm{S}})=\{\mathrm{S} \cdot \tilde{\mathrm{S}}\}$ or $B(\mathrm{~S}, \sigma)=\mathrm{S} \sigma$, with (25.6.ii) - (25.6.iii), we obtain

$$
\mathrm{j}^{k}[\{\mathrm{~S} \cdot \mathrm{S}\}]=\mathrm{j}^{k}[\{(\mathrm{D} \lambda) \cdot(\mathrm{D} \lambda)\}], \quad \mathrm{j}^{k}[\mathrm{~S} \sigma]=\mathrm{j}^{k}[(\mathrm{D} \lambda) \psi] .
$$

However, by Lemma 20.1(ii), (25.5) and (25.6.i), the $\approx$ equivalences in Theorem 21.1 imply equalities of $k$-jets at $t=0$. Since the left-hand sides in (19.1) all vanish due to our choice of $f, h, x, y, z, p, q$ and $r,(21.1)$ and (21.3) thus yield

$$
\begin{aligned}
& \text { i) }[4 \mathrm{D}\{(\mathrm{D} \lambda) \cdot(\mathrm{D} \lambda)\}+(\mathrm{D} \lambda) \psi]^{(k)} \\
& \text { ii) }[\zeta]^{(k)}=-\left[y \tau_{b}\right]^{(k)} \text {, }
\end{aligned}
$$

where $[\ldots]^{(k)}$ denotes the $k$ th derivative of $\ldots$ at $t=0$.

[In case (25.1), we leave formula (25.8) unchanged, as its ingredients all refer to $\mathfrak{g}$, and modify (25.4) - (25.7) as follows. In (25.4), (25.6) and (25.7), $2 \operatorname{Re} \psi$ should appear, rather than $\psi$. In the first equality of $(25.7),\{\cdot\}_{\mathbb{R}}$ has to be used instead of $\{\cdot\}$, while the "multiplications" should be replaced by their $\mathfrak{g}_{\mathbb{R}}$ counterparts (and $\psi$ by $2 \operatorname{Re} \psi$ ). Since $\mathrm{D} \lambda$ is $\mathbb{C}$-bilinear, Remarks 13.2 and $13.3(\mathrm{v})$ allow us to rewrite the modified right-hand sides in $(25.7)$ as $2 \mathrm{j}^{k}[\operatorname{Re}\{(\mathrm{D} \lambda) \cdot(\mathrm{D} \lambda)\}]$ and $\mathrm{j}^{k}[(\mathrm{D} \lambda) \psi]$, with both operations now referring to the complex Lie algebra $\mathfrak{g}$.]

From $(25.7)$ and $(25.2 . \mathrm{i})$ we get $-\Delta[\mathrm{S}]^{(k)}=4 \mathrm{D}[\{(\mathrm{D} \lambda) \cdot(\mathrm{D} \lambda)\}]^{(k)}+[(\mathrm{D} \lambda) \psi]^{(k)}$, so that, by $(25.8 . \mathrm{i}), \Delta[\mathrm{S}]^{(k)}=-\mathrm{D}\left[U \tau_{a}+h x p \tau_{a}+V \theta_{a}+W \mu_{a}+x p \tau_{b}\right]^{(k)}$. Next, (12.1) and (16.8) give $-\Delta(\mathrm{D} \lambda)=\mathrm{D}\left[\left(n^{2} y+2 z\right) \theta_{a}+(2 y+z) \mu_{a}-h y \tau_{a}-y \tau_{b}\right]$, as $\mathrm{D} \beta=0$, cf. (9.6.c), and $c$ may be replaced with $h a+b$. Adding the former equality to $[\ldots]^{(k)}$ of the latter, and using equations (ii), (iv), (v) in (19.1), we obtain $\Delta[\mathrm{S}-\mathrm{D} \lambda]^{(k)}=-\left[(y+x p) \tau_{b}\right]^{(k)}$. Both sides here must vanish as a consequence of (12.5.b), since one lies in $\Delta(\mathcal{S})$ and the other, due to (12.2), in $\operatorname{Ker} \Delta$. The resulting relation $\left[(y+x p) \tau_{b}\right]^{(k)}=0$, along with (25.6.i) and the fact that, by (19.3), $y+x p \neq 0$ at $t=0$, yields (25.4.i). Vanishing of $\Delta[\mathrm{S}-\mathrm{D} \lambda]^{(k)}$ implies in turn that $[\mathrm{S}-\mathrm{D} \lambda]^{(k)}=0$, as $\Delta$ is injective on $\Delta(\mathcal{S})$ (see (12.5.c)), while $[\mathrm{S}-\mathrm{D} \lambda]^{(k)} \in \Delta(\mathcal{S})$ according to (25.2.iii). Now (25.4.ii) follows from (25.6.ii). Finally, (25.4.i) and (25.8.ii) give $[\zeta]^{(k)}=0$. The definition of $\zeta$ in Theorem 21.1, 
combined with the equality $[\sigma]^{(k)}=[8\{\mathrm{D} \cdot(\mathrm{D} \lambda)\}+4\{(\mathrm{D} \lambda) \cdot(\mathrm{D} \lambda)\}]^{(k)}$ (obvious from (25.2.ii) and (25.4.ii)), shows that $[\sigma]^{(k)}=[\psi]^{(k)}$. Thus, (25.4.iii) is immediate from (25.6.iii), completing the induction step, and proving (25.3).

[In case (25.1), the last paragraph requires only minor changes. The righthand side of the formula in the first line is $\mathbb{C}$-bilinear, since so is $\mathrm{D} \lambda$, even if $2 \operatorname{Re} \psi$ is used instead of $\psi$. (See Remarks 13.2 and 13.3(v).) Thus, applying Remark 13.3(viii) to the left-hand side, which now reads $-\Delta_{\mathbb{R}}[\mathrm{S}]^{(k)}$, we conclude that $[\mathrm{S}]^{(k)}$ is $\mathbb{C}$-bilinear, and Remark 13.3 (vii) allows us to replace $\Delta_{\mathbb{R}}$ with $\Delta$. Also, 2 Re must precede the right-hand sides of the last two equalities.]

Our fixed real-analytic curve $[0, \delta) \ni t \mapsto \mathrm{S}(t) \in \mathcal{S}$ with $\mathrm{S}(0)=0$, lying entirely in $\boldsymbol{H}^{-1}(0)$, thus satisfies relations (25.3), in which all summands and factors, along with $\xi=(a, a) / n$, are scalar or vector-valued real-analytic functions of the variable $t$. It now follows that $\xi$ vanishes identically in $[0, \delta)$. In fact, otherwise, for some $\delta^{\prime} \in(0, \delta)$, one would have $h \xi \neq 0$ at all $t \in\left(0, \delta^{\prime}\right]$ and $h^{2} / \xi \rightarrow 0$ as $t \rightarrow 0$ since, by (19.3) - (19.4), $h$ is an analytic function of the variable $\xi$ with $h=0 \neq d h / d \xi$ at $\xi=0$, while $\xi=0$ at $t=0$ according to (25.5). Consequently, $h^{2} / \xi$ would be nonconstant. Yet it must be constant: by (24.1), its values are all rational. The resulting contradiction proves that $\xi=0$ identically. (Note that (25.3.i) implies (22.1), and so Lemma 24.1 may be applied here to $a=a(t)$, for any $t \in\left(0, \delta^{\prime}\right]$.)

Since $\xi=0$ on $[0, \delta)$, the functions $h, x, y, z, p, q, r$ and $f$, depending on $t$ via $\xi$, are all constant and their values are given by (19.3). Thus, (25.3.ii) amounts to $\mathrm{S}=\mathrm{D} \eta_{a}$ for all $t$, as in (22.3), with $a^{2}=0$, completing the proof.

Remark 25.2. Here is why (S, $\sigma)-\boldsymbol{I}_{a}[\mathbf{v}]$ lies in the image of $\boldsymbol{L}$ (as claimed in the lines following (2.18), with $\mathrm{S}, \sigma, a, \mathbf{v}$ depending on $t)$. By $(2.5),(\mathrm{S}, \sigma)-\boldsymbol{I}_{a}[\mathbf{v}]=$ $(\mathrm{S}-\mathrm{D} \lambda, \sigma-\psi)$, while $\boldsymbol{L}(\mathrm{S}, \sigma)=(\Delta \mathrm{S}, \sigma)$ (see Remark 11.3), and $\mathrm{S}-\mathrm{D} \lambda \in \Delta(\mathcal{S})$ in view of (25.2.iii).

\section{Proof of Theorem 22.3}

We have the following consequence of Lemma 25.1.

Corollary 26.1. Under the assumptions listed at the beginning of Section 25, the set $\mathcal{C}=\mathrm{D}+\mathcal{L}$ of Einstein connections in $\mathfrak{g}$, defined by (22.3), contains all weakly-Einstein connections sufficiently close to $\mathrm{D}$. This is the case for $\mathfrak{g}_{\mathbb{R}}$ in (25.1) as well.

Proof. By (11.8.ii), weakly-Einstein connections in $\mathfrak{g}$ form the set $\mathrm{D}+\mathcal{Z}$, where $\mathcal{Z}=\boldsymbol{H}^{-1}(0)$. Lemma 25.1 states that the hypotheses, and hence the conclusion, of Corollary 4.2 are satisfied by $\mathcal{L}$ in $(22.3)$ and $\mathcal{Z}$, both of which are algebraic sets due to Theorem 22.2(iv) and the definition (11.2.i) of $\boldsymbol{H}$.

Theorem 22.3 is now immediate. First, Corollary 26.1 applied to the triple $(\mathfrak{s u}(l, j), \mathbb{R}, \mathrm{i})$ in (16.1) implies that all Einstein connections sufficiently close to 
$\mathrm{D}$ lie in $\mathcal{C}$. Secondly, for $j=0$ and $l=n \geq 3$ one has $\mathcal{C}=\{\mathrm{D}\}$, as 0 is the only element $a \in \mathfrak{s u}(n)$ with $a^{2}=0$. In other words, the Levi-Civita connection $\mathrm{D}$ of the Killing form $\beta$ is isolated among Levi-Civita connections of left-invariant Riemannian Einstein metrics on $\mathrm{SU}(n)$. Rephrased in terms of metrics (see Remark 6.2), this amounts to Theorem 22.3.

\section{Complex Witt bases}

The following lemma uses Witt's theorem [2, Chapter 13] to evaluate the dimensions of some Lie algebras and manifolds.

Lemma 27.1. Let $e_{1}, \ldots, e_{k}$ be a basis of a totally null complex subspace $\mathcal{V}$ in the space $\mathbb{C}^{n}$ endowed with the standard sesquilinear Hermitian inner product $\langle$,$\rangle of the$ sign pattern formed by $l$ pluses and $j$ minuses, where $l \geq j \geq k$.

(a) $\mathbb{C}^{n}$ has a basis $e_{1}, \ldots, e_{k}, \hat{e}_{1}, \ldots, \hat{e}_{k}, u_{2 k+1}, \ldots, u_{n}$ containing $e_{1}, \ldots, e_{k}$ such that, for all $p, q, r, s$ in the appropriate ranges, $\left\langle e_{r}, e_{s}\right\rangle=\left\langle\hat{e}_{r}, \hat{e}_{s}\right\rangle=\left\langle e_{r}, u_{p}\right\rangle=$ $\left\langle\hat{e}_{r}, u_{p}\right\rangle=0,\left\langle e_{r}, \hat{e}_{s}\right\rangle=\delta_{r s},\left\langle u_{p}, u_{p}\right\rangle \in\{1,-1\}$, and $\left\langle u_{p}, u_{q}\right\rangle=0$ if $p \neq q$.

(b) $\operatorname{dim} \mathfrak{h}=k^{2}$ for the Lie algebra $\mathfrak{h}$ consisting of all $a \in \mathfrak{s u}(l, j)$ with $a\left(\mathbb{C}^{n}\right) \subset \mathcal{V}$.

(c) Every $a \in \mathfrak{s u}(l, j)$ with $a^{2}=0$ is $\mathrm{SU}(l, j)$-conjugate to ta, for all $t \in(0, \infty)$, so that 0 lies in the closure of the $\mathrm{SU}(l, j)$-orbit of $a$.

(d) $\operatorname{dim} \mathfrak{k}=(n-k)^{2}+2 k^{2}-1$ for the Lie algebra $\mathfrak{k}=\{a \in \mathfrak{s u}(l, j): a(\mathcal{V}) \subset \mathcal{V}\}$.

(e) The set $\mathrm{N}_{l, j, k}$ of all $k$-dimensional totally null complex subspaces of $\mathbb{C}^{n}$ is a manifold, $\operatorname{dim} \mathrm{N}_{l, j, k}=(2 n-3 k) k$, and the action of $\mathrm{SU}(l, j)$ on $\mathrm{N}_{l, j, k}$ is transitive.

(f) Elements $a \in \mathfrak{s u}(l, j)$ with $a^{2}=0$ form exactly $(j+1)((j+2) / 2 \mathrm{SU}(l, j)$-conjugacy classes, classified by the values of rank $a \in\{0,1, \ldots, j\}$ and the positive index $\operatorname{ind}_{+} a$ of the Hermitian form $\mathrm{i}\langle a(\cdot), \cdot\rangle$, with $\operatorname{ind}_{+} a \in\{0,1, \ldots$, rank $a\}$.

Proof. Since $\langle$,$\rangle descends to a (nondegenerate) Hermitian inner product in \mathcal{V}^{\perp} / \mathcal{V}$, we may choose $u_{2 k+1}, \ldots, u_{n}$ representing an orthonormal basis of $\mathcal{V}^{\perp} / \mathcal{V}$, and then define $\tilde{e}_{1}, \ldots, \tilde{e}_{k} \in \mathbb{C}^{n}$ by requiring $\left\langle\cdot, \tilde{e}_{1}\right\rangle \ldots,\left\langle\cdot, \tilde{e}_{k}\right\rangle$ to be the first $k$ elements of basis of $\left[\mathbb{C}^{n}\right]^{*}$ dual to a basis for which $e_{1}, \ldots, e_{k}, u_{2 k+1}, \ldots, u_{n}$ are the initial $n-k$ vectors. Setting $\hat{e}_{r}=\tilde{e}_{r}-\sum_{s=1}^{k}\left\langle\tilde{e}_{r}, \tilde{e}_{s}\right\rangle e_{s} / 2$, we obtain (a).

Skew-adjointness of $a \in \mathfrak{h}$, combined with the relation $a\left(\mathbb{C}^{n}\right) \subset \mathcal{V}$, gives $\mathcal{V}^{\perp} \subset \operatorname{Ker} a$. Thus, elements $a$ of $\mathfrak{h}$ are characterized by

$$
a e_{r}=a u_{p}=0, \quad a \hat{e}_{r}=\sum_{s=1}^{k} X_{r s} e_{s}, \quad \text { where } X=\left[X_{r s}\right] \in \mathfrak{u}(k),
$$

the requirement that the $k \times k$ matrix with the entries $X_{r s}$ be skew-Hermitian expressing here skew-adjointness of $a \in \mathfrak{h}$. (As $a\left(\mathbb{C}^{n}\right) \subset \mathcal{V} \subset \mathcal{V}^{\perp} \subset \operatorname{Ker} a$, Remark 15.1(i) implies that $a$ is traceless.) Since $\operatorname{dim} \mathfrak{u}(k)=k^{2}$, (b) follows.

Given $a \in \mathfrak{s u}(l, j)$ with $a^{2}=0$, the image $a\left(\mathbb{C}^{n}\right)$ is totally null. Thus,

$e_{1}, \ldots, e_{k} \operatorname{span} a\left(\mathbb{C}^{n}\right)$ for $k=\operatorname{rank} a$ and some basis as in (i), 
which yields (27.1) with some $X$. Replacing each $e_{r}$ by $t^{-1 / 2} e_{r}$ and $\hat{e}_{r}$ by $t^{1 / 2} \hat{e}_{r}$ we obtain a new basis with (27.2), in which $X$ now represents $t a$, proving (c).

Next, $a \in \mathfrak{k}$ if and only if $a$ has, in the basis with (i), the block matrix form

$$
\left[\begin{array}{ccc}
X & Y & T \\
0 & H & -Y^{*} \\
0 & 0 & -X^{*}
\end{array}\right]
$$

Here $T=-T^{*} \in \mathfrak{u}(k)$ and $H=-H^{*} \in \mathfrak{u}(l-k, j-k)$, while ()$^{*}$ denotes three versions of the Hermitian transpose. The zero submatrices reflect the inclusions $a(\mathcal{V}) \subset \mathcal{V}$ and $a\left(\mathcal{V}^{\perp}\right) \subset \mathcal{V}^{\perp}$ (the latter due to skew-adjointness of $a$ ). This gives the formula in (d), with $X, Y, T, H$ and tracelessness of $a$ (which is a separate condition) contributing $2 k^{2}, 2(n-2 k) k, k^{2},(n-2 k)^{2}$ and -1 to the total.

Transitivity in (e) is obvious from (a). Thus, $\mathrm{N}_{l, j, k}$ is a manifold. By (d), $\operatorname{dim} \mathrm{N}_{l, j, k}=\operatorname{dim} \mathrm{SU}(l, j)-\operatorname{dim} \mathfrak{k}=n^{2}-1-\left[(n-k)^{2}+2 k^{2}-1\right]$, as claimed in (e).

Finally, given $a \in \mathfrak{s u}(l, j)$ with $a^{2}=0$, the image $a\left(\mathbb{C}^{n}\right)$ is totally null. We thus have (27.2), and hence (27.1) for some $X$. Another basis as in (27.2) arises when all $e_{r}$ and $\hat{e}_{r}$ are replaced by $e_{r}^{\prime}$ and $\hat{e}_{r}^{\prime}$, where $e_{r}^{\prime}=\sum_{s=1}^{k} W_{r s} e_{s}$ and $\hat{e}_{r}^{\prime}=\sum_{s=1}^{k} Z_{r s} \hat{e}_{s}$ for any invertible complex $k \times k$ matrix $Z$ and its inverse conjugate transpose $W$. The matrix playing the role of $X$ in (27.1) for $a$ and the new basis is $Z X Z^{*}$, where $Z^{*}=W^{-1}$ is the conjugate transpose of $Z$. Since $X \in \mathfrak{u}(k)$ is orthonormally diagonalizable, a suitable choice of $Z \in \mathfrak{u}(k)$ will render $Z X Z^{*}$ diagonal, with imaginary entries. Furthermore, rescaling each $e_{r}$ and $\hat{e}_{r}$ as in the line following (27.2), with $t>0$ possibly depending on $r$, allows us to assume that the imaginary diagonal entries are all $\pm \mathrm{i}$ or 0 . Combined with transitivity in (e), this gives (f).

Remark 27.2. The analog of (f) in Lemma 27.1 for $\operatorname{SL}(n, \mathbb{F}$ ) (where $\mathbb{F}$ is $\mathbb{R}, \mathbb{C}$ or $\mathbb{H})$, rather than $\mathrm{SU}(l, j)$, is simpler: elements $a$ of $\mathfrak{s l}(n, \mathbb{F})$ with $a^{2}=0$ form $[n / 2]+1 \mathrm{SL}(n, \mathbb{F})$-conjugacy classes, classified by the value of rank $a$, ranging over $\{0,1, \ldots, j\}$. In fact, if $k=\operatorname{rank} a$, a basis

$$
a e_{1}, \ldots, a e_{k}, e_{1}, \ldots, e_{k}, u_{2 k+1}, \ldots, u_{n}
$$

where $a e_{1}, \ldots, a e_{k}, u_{2 k+1}, \ldots, u_{n}$ span Ker $a$, gives $a$ a canonical matrix form.

Remark 27.3. Assertion (c) of Lemma 27.1 remains valid if one replaces $\mathrm{SU}(l, j)$ with any of the other groups in (1.1). To see this, one may use, instead of (27.3), the basis $\operatorname{tae}_{1}, \ldots, \operatorname{tae}_{k}, e_{1}, \ldots, e_{k}, u_{2 k+1}, \ldots, u_{n}$.

Condition (c) in Lemma 27.1 is also an immediate consequence of (f) (and similarly for $\mathrm{SU}(l, j))$. We state it separately for easy reference. 


\section{Proofs of Theorem 22.2, second part, and Theorem 22.4}

By (11.1), $\mathcal{C} \subset \mathcal{E} \subset \mathcal{W} \subset \mathrm{D}+\mathcal{S}$, which reduces proving Theorem 22.2(v) to establishing relative openness of $\mathcal{C}$ in $\mathcal{W}$ or, equivalently, showing that every point $\mathrm{D}+\mathrm{S}$ of $\mathcal{C}$ has a neighborhood in the affine space $\mathrm{D}+\mathcal{S}$ which does not intersect $\mathcal{W} \backslash \mathcal{C}$. To this end let us suppose that, on the contrary, some $\mathrm{D}+\mathrm{S} \in \mathcal{C}$ is the limit as $k \rightarrow \infty$ of a sequence $\mathrm{D}+\mathrm{S}_{k}$ in $\mathcal{W} \backslash \mathcal{C}$. By Remark 27.3, any neighborhood of $\mathrm{D}$ contains an adjoint-action image of $\mathrm{D}+\mathrm{S}$ and, with it, the images of $\mathrm{D}+\mathrm{S}_{k}$ for all large $k$. Thus, some sequence in $\mathcal{W} \backslash \mathcal{C}$ converges to $\mathrm{D}$, which contradicts Corollary 26.1.

The set $\mathcal{C}$, being connected (since so is the cone $\mathcal{P}=\left\{a \in \mathfrak{g}: a^{2}=0\right\}$ ), as well as closed and relatively open in both $\mathcal{E}$ and $\mathcal{W}$ (by (iv) - (v)), must be a connected component of both, which proves (vi). Assertion (viii) is in turn immediate from Lemma 27.1(f) and Remark 27.2.

All of the above remains valid for the underlying real Lie algebra of $\mathfrak{s l}(n, \mathbb{C})$.

The next result trivially implies (vii) in Theorem 22.2, as well as Theorem 22.4; for the latter, we also use Remark 13.3(ii)-(iv), the final clause of Corollary 26.1, and the preceding one-line paragraph.

In the remainder of this section $(\mathfrak{g}, \mathbb{F}, \varepsilon)$ denotes one of the triples $(16.1)$, for $n=l+j \geq 3$. All manifolds and mappings are assumed $\mathbb{F}$-analytic, with the term 'real/complex' indicating the appropriate choice between $\mathbb{F}=\mathbb{R}$ or $\mathbb{F}=\mathbb{C}$. The symbol [ ] stands for the integer part, and $\mathrm{Gr}_{k}^{n}$ for the real/complex Grassmann manifold of all $k$-dimensional real/complex vector subspaces of $\mathbb{F}^{n}$.

The polynomial bijective correspondence in Theorem 22.2(ii) reduces the description of $\mathcal{C}$ to determining the structure of the cone $\mathcal{P}=\left\{a \in \mathfrak{g}: a^{2}=0\right\}$. We achieve the latter by providing the following nonsingular model for $\mathcal{P}$.

Theorem 28.1. There exist a connected $\mathbb{F}$-analytic manifold $\mathcal{M}$ with an open dense subset $\mathcal{M}^{\prime}$, and an $\mathbb{F}$-analytic mapping $\boldsymbol{Q}: \mathcal{M} \rightarrow \mathfrak{g}$, such that $\boldsymbol{Q}(\mathcal{M})=\mathcal{P}$ and $\boldsymbol{Q}$ sends $\mathcal{M}^{\prime}$ diffeomorphically onto a real/complex submanifold of $\mathfrak{g}$ contained in $\mathcal{P}$, while $\operatorname{dim}_{\mathbb{F}} \mathcal{M}=\left[n^{2} / 2\right]$ for $\mathfrak{g}=\operatorname{sl}(n, \mathbb{F})$ and $\operatorname{dim}_{\mathbb{R}} \mathcal{M}=2 l j$, if $\mathfrak{g}=\operatorname{su}(l, j)$, or $\operatorname{dim}_{\mathbb{R}} \mathcal{M}=4\left[n^{2} / 8\right]$, if $\mathfrak{g}=\operatorname{sl}(n / 2, \mathbb{H})$.

Proof. For triples other than $\mathfrak{s l}(n / 2, \mathbb{H}), \mathbb{R}, 1)$, we define the integer $k$ by $k=$ $[n / 2]$ if $\mathfrak{g}=\operatorname{sl}(n, \mathbb{F})$ and $k=j$ if $\mathfrak{g}=\operatorname{su}(l, j)$. As $j \leq l$, in the latter case $k$ is the maximum dimension of a totally null complex subspace in $\mathbb{C}^{n}$ endowed with the sesquilinear inner product mentioned in Lemma 27.1.

Let $\mathcal{B}$ denote the compact connected $\mathbb{F}$-analytic manifold formed by all $\left(\mathcal{V}, \mathcal{V}^{\prime}\right) \in \operatorname{Gr}_{k}^{n} \times \operatorname{Gr}_{n-k}^{n}$ such that $\mathcal{V} \subset \mathcal{V}^{\prime}$ and, for $\mathfrak{g}=\operatorname{su}(l, j)$ only, $\mathcal{V}^{\prime}$ is the orthogonal complement of the complex totally null subspace $\mathcal{V}$. Thus, when $\mathfrak{g}=$ $\operatorname{sl}(n, \mathbb{F})$ and $n$ is odd, $\mathcal{B}$ is an $\mathbb{F P}^{n-k-1}$ bundle over $\operatorname{Gr}_{k}^{n}$, with the bundle projection $\left(\mathcal{V}, \mathcal{V}^{\prime}\right) \mapsto \mathcal{V}$ and the fibre $\mathrm{P}\left(\mathcal{V}^{\prime} / \mathcal{V}\right)$ over $\mathcal{V} \in \mathrm{Gr}_{k}^{n}$. For $\mathfrak{g}=\operatorname{sl}(n, \mathbb{F})$ and even $n$, we may identify $\mathcal{B}$ with $\operatorname{Gr}_{k}^{n}$, as $\mathcal{V}=\mathcal{V}^{\prime}$. Similarly, $\mathcal{V}$ uniquely 
determines $\mathcal{V}^{\prime}$ if $\mathfrak{g}=\mathrm{su}(l, j)$, which leads to the identification $\mathcal{B}=\mathrm{N}_{l, j, j}$, with $\operatorname{dim} \mathrm{N}_{l, j, k}=(2 l-j) j$ (as $n=l+j$, cf. Lemma 27.1(e)). Consequently, depending on whether $\mathfrak{g}=\operatorname{sl}(n, \mathbb{F})$ and $n$ is even, or $\mathfrak{g}=\operatorname{sl}(n, \mathbb{F})$ and $n$ is odd, or $\mathfrak{g}=\operatorname{su}(l, j)$,

$$
\operatorname{dim}_{\mathbb{F}} \mathcal{B}=k^{2} \text {, or } \operatorname{dim}_{\mathbb{F}} \mathcal{B}=(k+2) k \text {, or } \operatorname{dim}_{\mathbb{R}} \mathcal{B}=(2 l-j) j .
$$

We denote by $\mathcal{M}$ the total space of the real/complex vector bundle over $\mathcal{B}$ with the fibre $\mathcal{F}$ over any $\left(\mathcal{V}, \mathcal{V}^{\prime}\right) \in \mathcal{B}$ consisting of all $a \in \mathfrak{g}$ such that the image of $a$ as a linear endomorphism of $\mathbb{R}^{n}$ or $\mathbb{C}^{n}$ is contained in $\mathcal{V}$, and its kernel contains $\mathcal{V}^{\prime}$. As the inclusion $\mathcal{V} \subset \mathcal{V}^{\prime}$ then gives $a^{2}=0$, an obvious surjective mapping $\boldsymbol{Q}: \mathcal{M} \rightarrow \mathcal{P}$ is defined by requiring the $\boldsymbol{Q}$-image of a fibre element $a$ at any $\left(\mathcal{V}, \mathcal{V}^{\prime}\right) \in \mathcal{B}$ to be $a$ itself. In all cases, $\operatorname{dim}_{\mathbb{F}} \mathcal{F}=k^{2}$. In fact, if $\mathfrak{g}=\operatorname{sl}(n, \mathbb{F})$, one may view $\mathcal{F}$ as the space of all linear operators $\mathbb{F}^{n} / \mathcal{V}^{\prime} \rightarrow \mathcal{V}$, while for $\mathfrak{g}=\operatorname{su}(l, j)$ we can use Lemma 27.1(b).

The vector bundle just described is an $\mathbb{F}$-analytic subbundle of the trivial bundle with the fibre $\mathfrak{g}$ over $\mathcal{B}$, since it is the kernel of the obvious $\mathbb{F}$-analytic bundle morphism, the rank of which is constant as $\operatorname{dim}_{\mathbb{F}} \mathcal{F}=k^{2}$. Thus, the manifold $\mathcal{M}$ and the mapping $\boldsymbol{Q}: \mathcal{M} \rightarrow \mathfrak{g}$ are both $\mathbb{F}$-analytic, while the dimension clause follows if one adds $\operatorname{dim}_{\mathbb{F}} \mathcal{F}=k^{2}$ to the dimensions in (28.1).

Let us now choose the open subset $\mathcal{M}^{\prime}$ of $\mathcal{M}$ to be the total space of a (non-vector) subbundle of the bundle $\mathcal{M}$, the fibre of which over any $\left(\mathcal{V}, \mathcal{V}^{\prime}\right) \in \mathcal{B}$ is the subset of the fibre of $\mathcal{M}$ formed by those $a$ that, in addition, have the maximum rank $k$. The diffeomorphic property of $\boldsymbol{Q}$ on $\mathcal{M}^{\prime}$ is then obvious, with the inverse mapping $\boldsymbol{Q}\left(\mathcal{M}^{\prime}\right) \rightarrow \mathcal{M}^{\prime}$ sending any maximum-rank $a$ to the fibre element $a$ at the point $\left(\mathcal{V}, \mathcal{V}^{\prime}\right) \in \mathcal{B}$ which is the image-kernel pair of $a$.

For the remaining triple $\mathfrak{s l}(n / 2, \mathbb{H}), \mathbb{R}, 1)$, one sets $k=[n / 4]$ and repeats the above definitions $\mathcal{M}$ and $\mathcal{F}$ verbatim, while that of $\mathcal{B}$ is modified: the constituents $\mathcal{V}, \mathcal{V}^{\prime}$ of any $\left(\mathcal{V}, \mathcal{V}^{\prime}\right) \in \mathcal{B}$ are, in addition, required to be quaternionic subspaces of $\mathbb{H}^{n / 2} \approx \mathbb{C}^{n}$. The argument used to prove (28.1), with $\mathbb{F}$ and $n$ replaced by $\mathbb{H}$ and $n / 2$, now shows that the "quaternionic" dimension of $\mathcal{B}$ equals $k^{2}$ or $(k+2) k$, depending on whether $n / 2$ is even or odd. Adding to either value $\operatorname{dim}_{\mathbb{H}} \mathcal{F}=k^{2}$, we obtain $\left[n^{2} / 8\right]$, so that $\operatorname{dim}_{\mathbb{R}} \mathcal{M}=4\left[n^{2} / 8\right]$. Finally, the discussion of the last paragraph applies to this case without any changes.

\section{Non-Einstein examples}

Not all weakly-Einstein connections arising in Theorem 22.1 are Einstein connections. For instance, the nonuple $(\xi, f, h, x, y, z, p, q, r)$, where

$$
\begin{aligned}
& \xi=(z+1) / z^{2}, \quad h=0, \quad x=1, \quad y=-z, \quad p=(z+2) / z, \\
& q=-n^{2}(z+2), \quad r=z+2, \quad f=(z+1)(2-z) / z^{2},
\end{aligned}
$$

will satisfy (19.1) with $(L, U, V, W)=\left(-2(z+1) / z, 0,\left(2-n^{2}\right) z,-z\right)$ in (19.2), if one chooses $z=-\left(n^{2}-2\right) /\left(n^{2}-1\right)$. In fact, all equalities in (19.1) except 
(19.1.iv), and the values of $L, U, W$, are easily verified by treating $z$ as arbitrary and substituting $n^{2} y+2 z$ for $V$ in (19.1.vii). We then also have $L+2=-2 / z$ and $z+1=1 /\left(n^{2}-1\right)$. Since $h=0<\xi$ in (29.1), the assumption (22.1) in Theorem 22.1 amounts to requiring that $(\varepsilon a)^{2}$ be a specific positive multiple of Id. Such $a \in \mathfrak{g}$ exists for any triple (16.1) as long as $n=l+j$ is even.

Consequently, (29.1) with $z=-\left(n^{2}-2\right) /\left(n^{2}-1\right)$ and any even $n \geq 3$ gives rise, via Theorem 22.1, to examples of unimodular connections $\nabla$ such that $\nabla \rho^{\nabla}=0$. They are not, however, Einstein connections, since $-4 \rho^{\nabla}=\beta+\psi$ is degenerate and nonzero. Namely, as explained below, a lies in the nullspace of $\beta+\psi($ and $a \neq 0$ by $(22.1)$ with $h=0 \neq \xi$ ), while $\langle\beta, \beta+\psi\rangle \neq 0$.

Specifically, since $\tau_{a}(a, \cdot)=\langle 2 c, \cdot\rangle, \theta_{a}(a, \cdot)=\langle\xi a, \cdot\rangle$, and $\mu_{a}(a, \cdot)=\langle d, \cdot\rangle$ by (16.3), using the three steps of Remark 15.2 to evaluate traces, we get

the images of $a$ under the endomorphisms (16.4) are $2 c, a, \xi a, d$, and the traces of the endomorphisms (16.4) equal $0, n^{2}-1, \xi,-\xi$.

Therefore $a$, if nonzero, is an eigenvector, for the eigenvalue $2 h p+\xi q+\left(h^{2}+\xi\right) r+$ $f+1$, of the linear endomorphism of $\mathfrak{g}$ corresponding to $-4 \rho^{\nabla}$ via (7.3), while the trace of that endomorphism is $\langle\beta, \beta+\psi\rangle=\left(n^{2}-1\right) q+(r-f) \xi$, with $\langle$,$\rangle as in$ (8.1.i). The claim concerning the eigenvalue is obvious here from (29.2), along with the equalities $b=0$ and $d=\left(h^{2}+\xi\right) a$, immediate from the assumption $c=h a$ (that is, (22.1)) and (20.4) or, respectively, from (20.6) and Lemma 20.1(i).

The above conclusions about the eigenvalue and trace apply to $-4 \rho^{\nabla}=\beta+\psi$ for all connections $\nabla$ arising from Theorem 22.1. In the special case (29.1), with $z$ treated as arbitrary, the eigenvalue equals $(z+2)\left[1-\left(n^{2}-1\right)(z+1)\right] / z^{2}$, and so it is 0 due to our choice of $z$. Similarly, the trace is $\left(n^{2}-1\right)-\left(n^{2}+1\right)(z+1) \neq 0$.

Acknowledgments. The authors thank Wolfgang Ziller for helpful comments.

A part of A. Derdzinski's work on the paper was done at the Erwin Schrödinger International Institute for Mathematical Physics (ESI), Vienna, in July 2011, during the first week of the Workshop on Cartan Connections, Geometry of Homogeneous Spaces, and Dynamics, organized by Andreas Cap, Charles Frances and Karin Melnick.

Ś. R. Gal was partially supported by Polish MNiSW grant N N201 541738.

\section{References}

[1] D. V. Alekseevskiǔ, Homogeneous Riemannian spaces of negative curvature (in Russian). Mat. Sb. (N. S.) 96(138) (1975), 93-117, 168. Zbl 0325.53043 MR 0362145

[2] M. Berger, Géométrie, Vol. 4: Formes quadratiques, coniques et quadriques, CEDIC, Nathan Information, Paris 1977. Zbl 0423.51002 MR 0536873

[3] A. L. Besse, Einstein Manifolds, Ergeb. Math. Grenzgeb. (3) 10, Springer-Verlag, Berlin 1987. Zbl 0613.53001 MR 0867684 
[4] C. Böhm, M. Wang and W. Ziller, A variational approach for compact homogeneous Einstein manifolds. Geom. Funct. Anal. 14 (2004), no. 4, 681-733. Zbl 1068.53029 MR 2084976

[5] F. Bruhat and H. Cartan, Sur la structure des sous-ensembles analytiques réels. C. R. Acad. Sci. Paris 244 (1957), 988-990. Zbl 0081.17201

[6] J. E. D'Atri and W. Ziller, Naturally reductive metrics and Einstein metrics on compact Lie groups. Mem. Amer. Math. Soc. 18 (1979), no. 215, iii+72 pp. Zbl 0404.53044 MR 0519928

[7] A. Derdzinski and Ś. R. Gal, Curvature spectra of simple Lie groups, 2013 (preprint). Available at http://www.math.osu.edu/ derdzinski.1/preprints/css.pdf.

[8] G. W. Gibbons, H. Lü and C. N. Pope, Einstein metrics on group manifolds and cosets. J. Geom. Phys. 61 (2011), no. 5, 947-960. Zbl 1225.53047 MR 2776894

[9] J. Heber, Noncompact homogeneous Einstein spaces. Invent. Math. 133 (1998), 279352. Zbl 0906.53032 MR 1632782

[10] N. Koiso, Rigidity and stability of Einstein metrics - the case of compact symmetric spaces. Osaka J. Math. 17 (1980), no. 1, 51-73. Zbl 0426.53037 MR 0558319

[11] J. Lauret, Einstein solvmanifolds are standard. Ann. of Math. (2) 172 (2010), no. 3, 1859-1877. Zbl 1220.53061 MR 2726101

[12] C. LeBrun, Spaces of complex null geodesics in complex-Riemannian geometry. Trans. Amer. Math. Soc. 278 (1983), no. 1, 209-231. Zbl 0562.53018 MR 0697071

[13] M. L. Leite and I. Dotti de Miatello, Metrics of negative Ricci curvature on SL( $n, \mathbb{R})$, $n \geq 3$. J. Differential Geom. 17 (1982), 635-641. Zbl 0482.53037 MR 0683168

[14] J. Milnor, Singular points of complex hypersurfaces. Ann. of Math. Stud. 61, Princeton University Press, Princeton, NJ, 1968. Zbl 0184.48405 MR 0239612

[15] C. G. Moreira and M. A. S. Ruas, The curve selection lemma and the Morse-Sard theorem. Manuscripta Math. 129 (2009), no. 3, 401-408. Zbl 1173.58016 MR 2515490

[16] K. Olszak, On the Bochner conformal curvature of Kähler-Norden manifolds. Cent. Eur. J. Math. 3 (2005), no. 2, 309-317. Zbl 1119.53024 MR 2129909

[17] J. A. Schouten, Ricci-Calculus. Grundlehren Math. Wiss. 10, Springer-Verlag, Berlin 1954. Zbl 0057.37803 MR 0066025

[18] A. H. Wallace, Algebraic approximation of curves. Canad. J. Math. 10 1958, 242-278. Zbl 0081.37803 MR 0094355

Andrzej Derdzinski

Department of Mathematics, The Ohio State University, Columbus, OH 43210, USA

E-mail: andrzej@math.ohio-state.edu

Światosław R. Gal

Mathematical Institute,Wrocław University, pl. Grunwaldzki 2/4, 50-384 Wrocław, Poland

E-mail: Swiatoslaw.Gal@math.uni.wroc.pl 